Portland State University

PDXScholar

Spring 6-2-2014

\title{
Analyses of Bus Travel Time Reliability and Transit Signal Priority at the Stop-To-Stop Segment Level
}

Wei Feng

Portland State University

Follow this and additional works at: https://pdxscholar.library.pdx.edu/open_access_etds

Part of the Transportation Commons

Let us know how access to this document benefits you.

\section{Recommended Citation}

Feng, Wei, "Analyses of Bus Travel Time Reliability and Transit Signal Priority at the Stop-To-Stop Segment Level" (2014). Dissertations and Theses. Paper 1832.

https://doi.org/10.15760/etd.1831

This Dissertation is brought to you for free and open access. It has been accepted for inclusion in Dissertations and Theses by an authorized administrator of PDXScholar. Please contact us if we can make this document more accessible: pdxscholar@pdx.edu. 
Analyses of Bus Travel Time Reliability and Transit Signal Priority at the Stop-To-Stop Segment Level

by

Wei Feng

A dissertation submitted in partial fulfillment of the requirements for the degree of

Doctor of Philosophy

in

Civil and Environmental Engineering

Dissertation Committee:

Miguel Figliozzi, Chair

Robert Bertini

Christopher Monsere

James Strathman

Portland State University

2014 
(C) 2014 Wei Feng 


\begin{abstract}
Transit travel time is affected by many factors including traffic signals and traffic condition. Transit agencies have implemented strategies such as transit signal priority (TSP) to reduce transit travel time and improve service reliability. However, due to the lack of empirical data, the joint impact of these factors and improvement strategies on bus travel time has not been studied at the stop-to-stop segment level.
\end{abstract}

This study utilizes and integrates three databases available along an urban arterial corridor in Portland, Oregon. Data sources include stop-level bus automatic vehicle location (AVL) and automatic passenger count (APC) data provided by the Tri-County Metropolitan Transportation District of Oregon (TriMet), the Sydney Coordinated Adaptive Traffic System (SCATS) signal phase log data, and intersection vehicle count data provided by the City of Portland. Based on the unique collection and integration of these fine granularity empirical data, this research utilizes multiple linear regression models to understand and quantify the joint impact of intersection signal delay, traffic conditions and bus stop location on bus travel time and its variability at stop-to-stop segments. Results indicate that intersection signal delay is the key factor that affects bus travel time variability. The amount of signal delay is nearly linearly associated with intersection red phase duration. Results show that the effect of traffic conditions (volumes) on bus travel time varies significantly by intersection and time of day. 
This study also proposed new and useful performance measures for evaluating the effectiveness of TSP systems. Relationships between TSP requests (when buses are late) and TSP phases were studied by comparing TSP phase start and end times with bus arrival times at intersections. Results show that green extension phases were rarely used by buses that requested TSP and that most green extension phases were granted too late. Early green effectiveness (percent of effective early green phases) is much higher than green extension effectiveness. The estimated average bus and passenger time savings from an early green phase are also greater compared to the average time savings from a green extension phase. On average, the estimated delay for vehicles on the side street due to a TSP phase is less than the time saved for buses and automobiles on the major street.

Results from this study can be used to inform cities and transit agencies on how to improve transit operations. Developing appropriate strategies, such as adjusting bus stop consolidation near intersections and optimizing bus operating schedules according to intersection signal timing characteristics, can further reduce bus travel time delay and improve TSP effectiveness. 


\section{ACKNOWLEDGEMENTS}

I would like to express my deepest appreciation to my adviser Professor Miguel Figliozzi for providing patient and detailed guidance and support throughout my degree program and dissertation process. I would also like to thank my previous adviser and committee member Professor Robert Bertini for his considerate guidance throughout my program and his invaluable comments on my dissertation. In addition, I am honored to have Professors Christopher Monsere and James Strathman as part of my committee. Their valuable comments made this research stronger.

I am deeply indebted to Steve Callas and David Crout from TriMet and Peter Koonce and Willie Rotchi from the City of Portland for providing us the unique data sources and their professional guidance. I would like to acknowledge Stephanie House and other Portland State University (PSU) writing center staffs for their help. I am also grateful to my colleagues and friends from the ITS lab and the PhT (PhD Students in Transportation) group at PSU for their invaluable dissertation comments, fruitful discussions and the stimulating and pleasant working environment.

Last but not least, I would like to thank my parents Yusheng Feng and Lanfeng Wang, my graduate studies would not have been possible without their extraordinary patience, love and support. 


\section{TABLE OF CONTENTS}

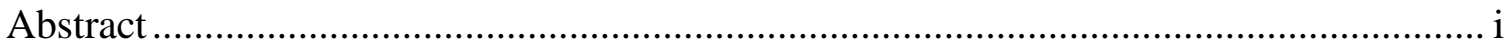

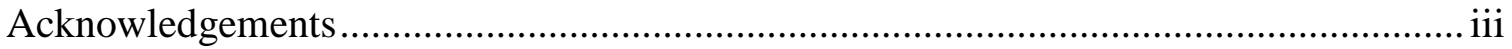

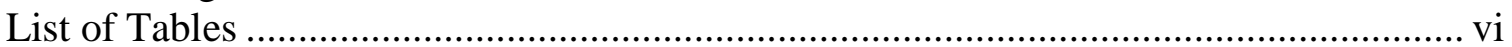

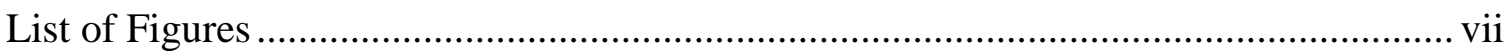

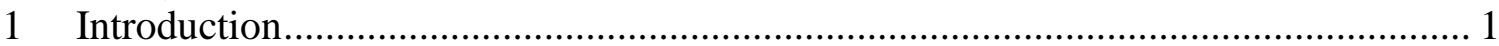

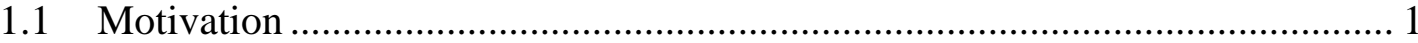

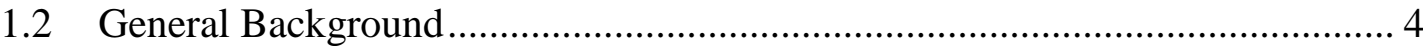

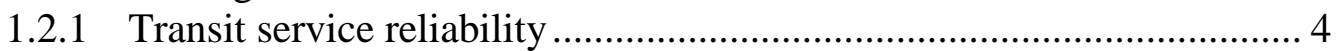

1.2.2 Factors affecting bus travel time and its variability ............................... 7

1.2.3 Transit signal priority performance evaluation ..................................... 14

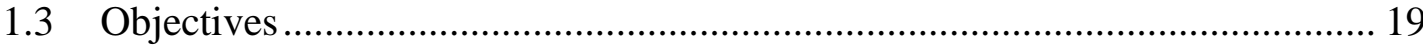

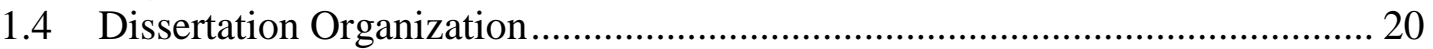

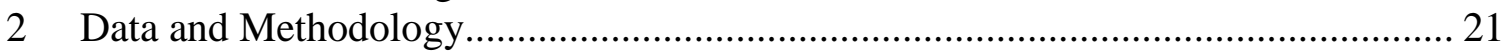

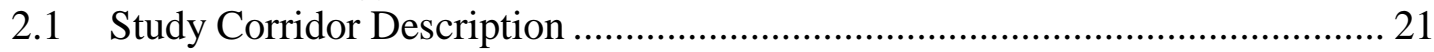

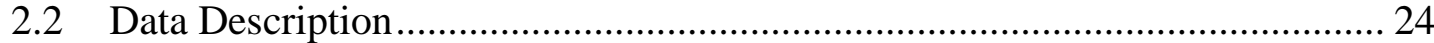

2.3 Research Methodology …………………………................................. 29

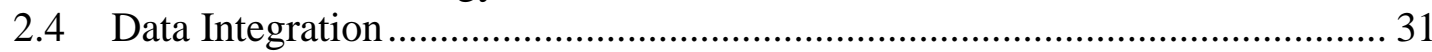

2.4.1 Bus stop activity attributes and stop-to-stop segment attributes .......... 32

2.4.2 Signal phase attributes ..................................................................... 33

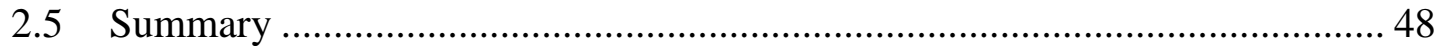

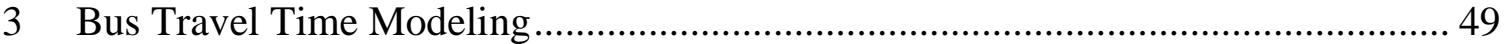

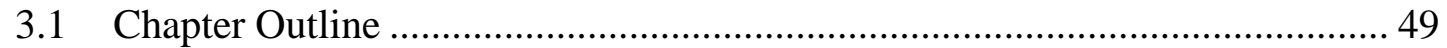

3.2 Variable Descriptions and Model Specifications ……………….................... 49

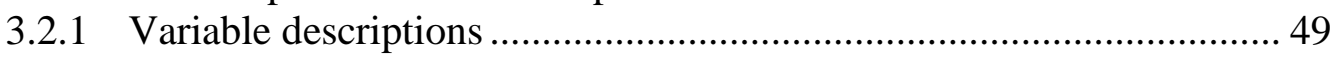

3.2.2 Model specifications .......................................................................... 56

3.2.3 Variable descriptive statistics ........................................................... 58

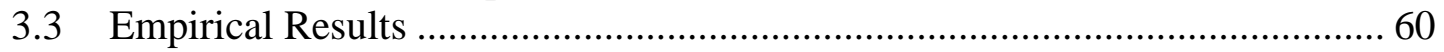

3.3.1 Far-side segments stop-to-stop travel time models ............................... 61

3.3.2 All segments arrival-to-arrival time model .............................................. 64

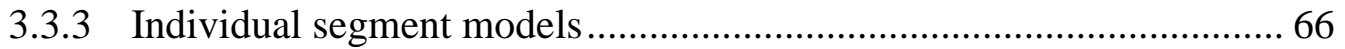

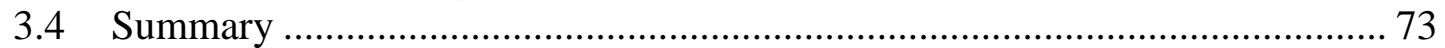

4 Transit Signal Priority Performance Evaluation ....................................................... 74

4.1 TSP System in Portland.............................................................................. 74

4.2 Relationships between TSP Phases and TSP Requests .................................... 78

4.2.1 Assumptions and definitions .......................................................... 78

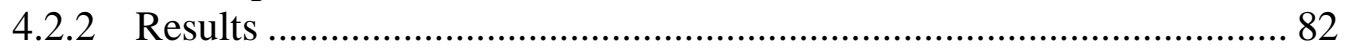

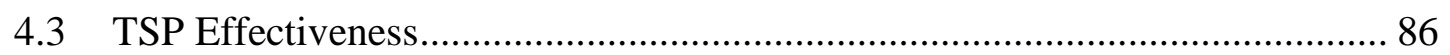

4.3.1 TSP request effectiveness .............................................................. 89

4.3.2 TSP phase effectiveness ……………………………………........ 92

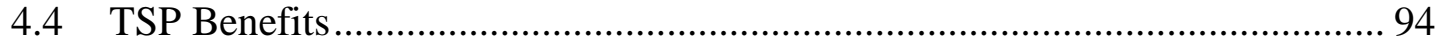

4.4.1 Estimated benefit per TSP request....................................................... 95 
4.4.2 Estimated benefit per TSP phase .................................................... 96

4.4.3 Time savings and delay between buses and other vehicles ................ 99

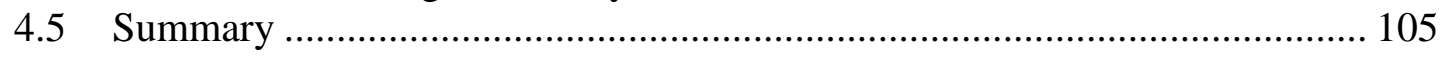

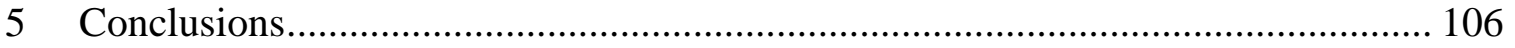

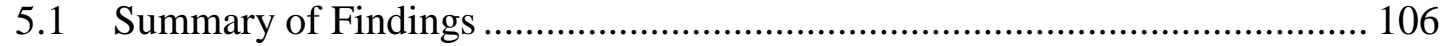

5.1.1 Bus stop-to-stop travel time modeling .......................................... 106

5.1.2 TSP performance evaluation ................................................... 111

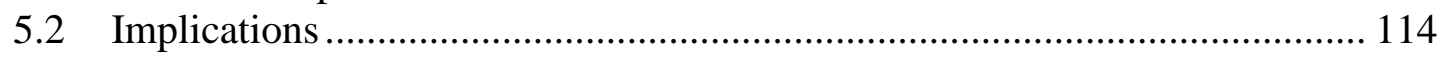

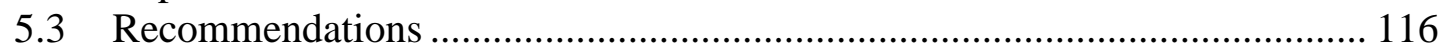

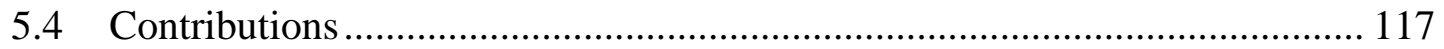

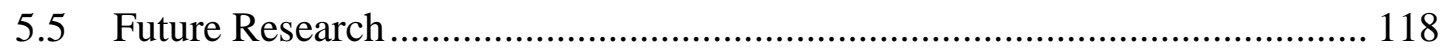

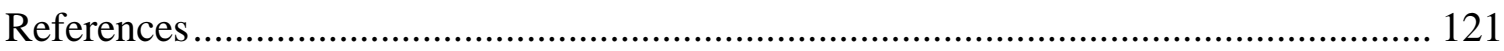

Appendix A: Bus Travel Speed Empirical Distributions........................................... 126

Appendix B: Algorithm to estimate bus stop-to-stop trip signal phase attributes ......... 130 


\section{LIST OF TABLES}

Table 1-1 Summary of Bus Travel Time Modeling Literature ..................................... 13

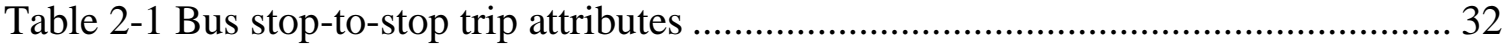

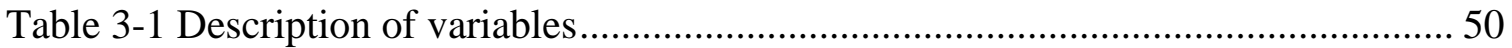

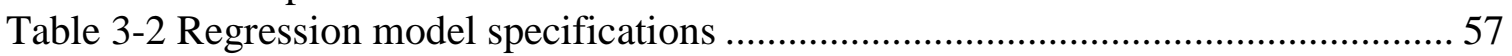

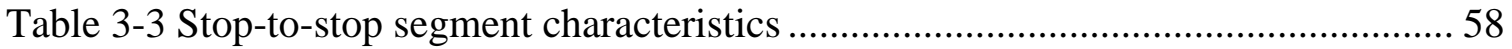

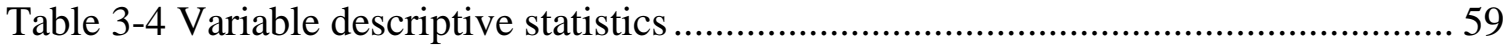

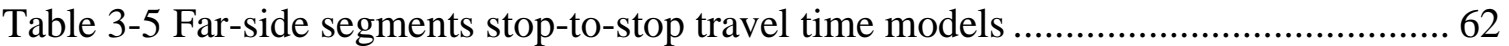

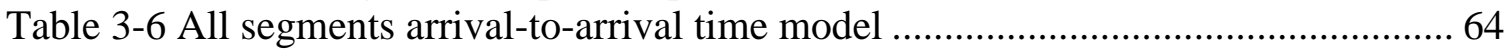

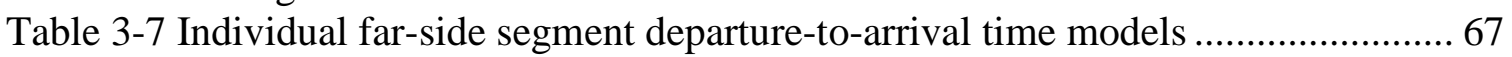

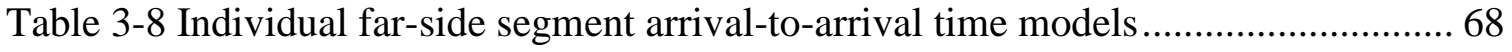

Table 3-9 Individual near-side segment arrival-to-arrival time models .......................... 69 


\section{LIST OF FIGURES}

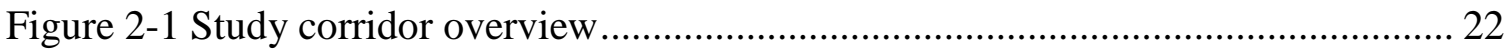

Figure 2-2 Median cycle length, red phase and green phase durations by direction ........ 22

Figure 2-3 Bus stop-to-stop segments that include SCATS signals ............................. 23

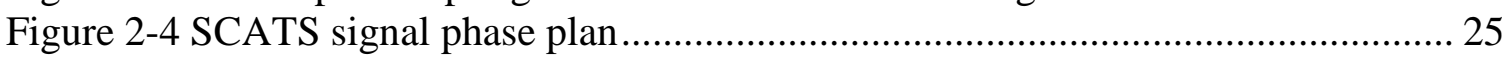

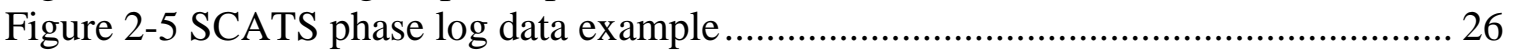

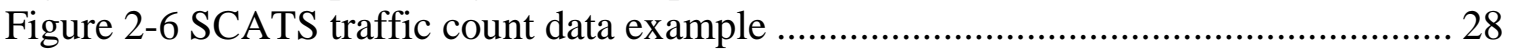

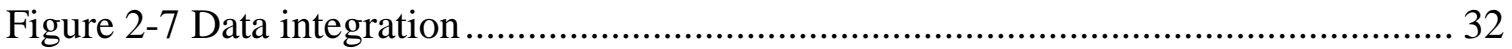

Figure 2-8 Time-space diagram of a bus that encounters a red signal delay ................... 38

Figure 2-9 Feasible bus stop-to-stop trip trajectories ............................................. 41

Figure 2-10 Feasible bus stop-to-stop trip trajectories with an early green phase........... 45

Figure 2-11 Feasible bus stop-to-stop trip trajectories with a green extension phase ...... 47

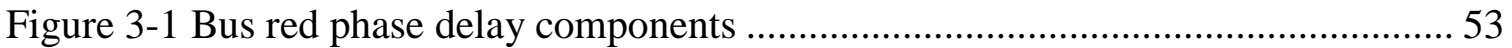

Figure 3-2 Red dummy variable coefficients vs. median red phase durations ................ 71

Figure 4-1 TSP system decision framework (Byrne et al., 2005) ................................ 75

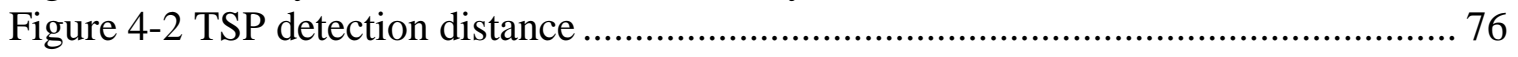

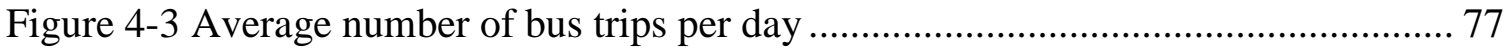

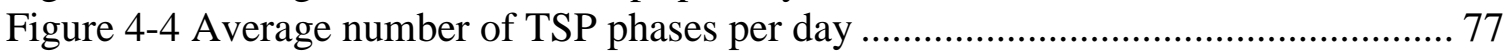

Figure 4-5 Relationships between TSP requests and TSP phases ................................. 78

Figure 4-6 Relationships between TSP requests and green extension phases ................. 80

Figure 4-7 Relationships between TSP requests and early green phases ...................... 81

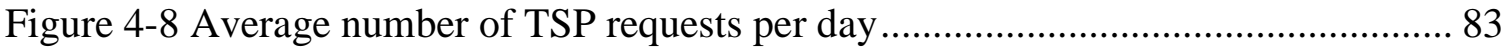

Figure 4-9 Average number of GE and EG phases per day.................................... 86

Figure 4-10 Detailed relationships between TSP requests and TSP phases .................... 87

Figure 4-11 Detailed relationships between TSP requests and green extension phases ... 88

Figure 4-12 Detailed relationships between TSP requests and early green phases ......... 88

Figure 4-13 Probability that a bus TSP request can benefit from a TSP phase ............... 90

Figure 4-14 Probabilities of TSP request outcomes for GE .................................... 91

Figure 4-15 Probabilities of TSP request outcomes for EG ...................................... 92

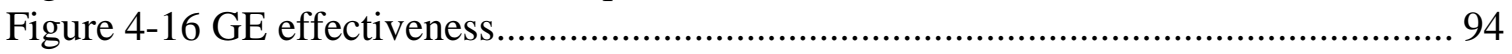

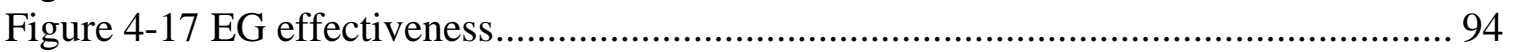

Figure 4-18 Estimated bus time savings per TSP request............................................ 96

Figure 4-19 Estimated total passenger time savings per TSP request ........................... 96

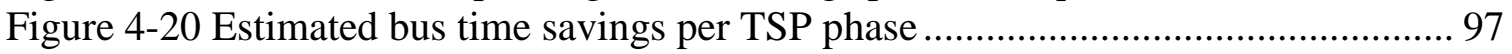

Figure 4-21 Estimated total passenger time savings per TSP phase .............................. 98

Figure 4-22 Illustration of major street time savings and side street delay ................... 100

Figure 4-23 Total passenger time savings and delay per TSP phase ............................ 101

Figure 4-24 Total passenger time savings and vehicle delays per second TSP phase.... 104 


\section{INTRODUCTION}

\subsection{Motivation}

Transit service reliability is important to both passengers and transit agencies. Slow and unreliable transit service may increase transit user costs in the short term and reduce transit mode share and ridership in the long term, which in turn may lead to higher levels of congestion, emissions, energy consumption, and car dependency in urban areas. In addition, bus travel time is important to schedulers because excessive variability forces schedulers to add excess slack or layover time to transit schedules. Therefore, transit agencies want to reduce bus travel time and its variability. In practice, there are many factors that affect bus travel time and its variability such as uncertain passenger demand, traffic conditions, driver behavior, signal delay at traffic lights and bus stop locations, road geometry, vehicle incidents/accidents, weather, etc. (Turnquist, 1981; Levinson, 1991; Ceder, 2007).

Although it is clear that traffic signals and traffic congestion impact transit vehicle delay, in practice, it is difficult to simultaneously isolate and quantify the impact of each of these factors along a corridor; there are no previous studies that have achieved this level of detail. Bus travel time delay at signalized intersections can be broken down into three components: waiting time for a red signal; acceleration and deceleration delay; and queuing effect delay. Traffic conditions can affect bus travel time near intersections in 
two ways: friction between buses and other vehicles due to merging or lane changing activities; vehicle queuing effect due to traffic congestion or traffic signals. Bus stop consolidation near intersections (near-side or far-side bus stops) can also affect bus travel time reliability. A Near-side stop is a transit stop located on the approach side of an intersection; a far-side stop is a transit stop located beyond an intersection (Kittelson \& Associates, Inc., 2013). Near-side bus stop consolidation can help some buses reduce signal delay by allowing them to serve passengers when waiting for a red signal; while far-side bus stops can reduce the conflict between buses and right-turn vehicles upstream of an intersection (Texas Transportation Institute, 1996; HCM, 2010; Kittelson \& Associates, Inc., 2013).

If the impacts of traffic signals, traffic conditions and bus stop location can be quantified, transit operators can prioritize investments or measures that tackle the main sources of delay, and propose appropriate strategies to reduce bus travel time and its variability. This study will focus on analyzing the joint impact of traffic signal delay, traffic conditions and bus stop location on bus travel time at the bus stop-to-stop segment level using empirical data. A bus stop-to-stop segment is a segment between two consecutive bus stops. This study will only focus on bus stop-to-stop segments that include a signalized intersection.

Transit agencies generally try to reduce bus travel time and improve service reliability by adopting various strategies. Transit signal priority (TSP) is one of the strategies that can help buses reduce travel time delay across an intersection. However, TSP is a complex 
process that involves traffic signal systems, transit vehicle detection systems and communication technologies. Although some studies evaluated TSP system performance using simulation techniques (Balke et al., 2000; Dion et al., 2004; Shalaby et al., 2003), practical assessment of TSP system showed that TSP benefits are not consistent across intersections (Albright and Figliozzi, 2012a) or across routes and time periods (Kimpel et al., 2005). In addition, previous studies did not have access to detailed TSP phase log data. Hence, another focus of this research will be to evaluate the TSP system effectiveness on reducing bus travel time delay across an intersection by integrating archived bus automatic vehicle location (AVL) and automated passenger count (APC) data with TSP phase log data.

The novelty of this research arises from the fine granularity of the analyses (intersection level) as well as the integration of new data sources including: bus AVL/APC data; Sydney Coordinated Adaptive Traffic System (SCATS) signal phase log data (including TSP phases); and SCATS intersection traffic count data. The unique integration of these three data sources make it possible to analyze the joint impact of traffic signal delay, traffic conditions and bus stop location on bus travel time reliability, and to evaluate TSP system performance at a high level of detail that is novel. The key contributions of this study include:

1. This study developed an algorithm that integrates the archived bus AVL/APC data, SCATS phase log data and intersection vehicle count data; 
2. this study simultaneously quantified the impacts of intersection signal delay, traffic conditions and bus stop location (near-side vs. far-side) on bus stop-to-stop segment travel time based on empirical data; and

3. this study proposed novel performance measures to evaluate the TSP system effectiveness at the stop-to-stop level.

\subsection{General Background}

\subsubsection{Transit service reliability}

Transit service reliability has been defined in a variety of ways (Turnquist and Blume, 1980; Abkowitz, 1978) and there is no single measure that can adequately address service quality. The most common measures of transit service reliability typically relate to schedule and headway adherence and travel time variation (Levinson, 1991; Turnquist, 1981; Strathman et al., 1999; Kimpel, 2001). On-time performance is commonly used by transit agencies to measure schedule adherence. It is defined as the percentage of buses that depart from a given location within a predetermined time window (Kittelson \& Associates, Inc., 2013). At the Tri-County Metropolitan Transportation District of Oregon (TriMet), the local transit provider for the Portland metropolitan area, a bus is defined as on-time if the bus departs from a time point bus stop no more than 1 minute early and 5 minutes late. Time point bus stops are specific point locations on bus routes from which vehicles are scheduled to depart at specified times (Kimpel, 2001). On-time performance is a valuable measure for low frequency bus service (scheduled headways 
longer than 10 minutes) and timed transfers. Headway coefficient of variation (standard deviation divided by mean) is used to measure headway adherence in high frequency service (headways less than 10 minutes) (Kittelson \& Associates, Inc., 2013). Poor schedule/headway adherence indicates deterioration of service reliability. Schedule/headway delay at the beginning of a route tends to propagate along the route and result in bus bunching (two buses running too close to each other) and large headway gaps, which increase total passenger delay and decrease passenger satisfaction. Bus travel time is defined as the time needed by a bus to travel between two points along a route. These points can be the beginning and ending terminal stations of the route (route level travel time), two consecutive time point bus stops (segment level travel time), or any two consecutive bus stops (bus stop-to-stop travel time).

Reductions in bus travel time and schedule/headway delay are expected to increase ridership and passenger satisfaction (Hensher et al., 2003; Murray and Wu, 2003; Vuchic, 2005). However, reducing scheduled travel time is challenging for transit agencies because changes in travel time have strong and often conflicting effects on schedule/headway reliability and the total costs for both passengers and transit agencies (Abkowitz and Engelstein, 1983; Abkowitz and Tozzi, 1987; Strathman et al., 2000). If the primary goal of a transit agency is to increase schedule/headway reliability, schedulers can add more slack time to bus stops. This increases the probability that a bus will depart from the stops on time by holding early arrival buses. However, this strategy increases passengers' total trip time and lowers bus operating speed, which increases both user cost and transit agency operating cost (Furth et al., 2006). If a transit agency’s 
primary goal is to minimize scheduled bus travel time, adding slack time will help transit agencies realize savings in recovery time and layover time, but can lead to decreases in schedule and headway reliability and a subsequent increase in user cost. The general guideline for establishing optimal bus travel times is supported by several transit planning software packages that set travel time between time points equal to the mean observed travel time (Furth et al., 2006; Kittelson \& Associates, Inc., 2013).

Automatic vehicle location (AVL) and automatic passenger count (APC) systems have been implemented by many transit agencies (Crout, 2007; Schweiger, 2003). With the availability of archived bus AVL and APC data, a substantial amount of statistical analyses were conducted to study how bus travel time and service reliability are affected by various impact factors and improvement strategies. Some studies analyzed bus travel time (Abkowitz and Engelstein, 1984; Bertini and El-Geneidy, 2004; El-Geneidy et al., 2011; Figliozzi and Feng, 2012; Slavin et al., 2013; Strathman et al., 2000), travel time delay (Diab and El-Geneidy, 2012; El-Geneidy et al., 2011, 2009; Strathman et al., 1999) and travel time coefficient of variation (El-Geneidy et al., 2010; Diab and El-Geneidy 2013) at the route level and time point segment level. Albright and Figliozzi (2012a) is the first study that analyzed bus travel time at the stop-to-stop segment level. Other studies analyzed bus schedule delay (Kimpel, 2001; Strathman et al., 1999), on-time performance (Strathman and Hopper, 1993; El-Geneidy and Surprenant-Legault, 2010; Rutherford and Watkins, 2011) and headway delay (Strathman et al., 1999; Kimpel 2001; El-Geneidy et al., 2010; Strathman et al., 2003; Kimpel et al., 2008; Figliozzi and Feng, 2012; Albright and Figliozzi, 2012c) at time point bus stops. Dueker et al. (2004) and 
Milkovits (2008) also studied bus dwell time for all bus stops. Most of the studies agree on some basic factors that affect bus travel time and schedule/headway reliability. These factors include distance, number of bus stops, number of signalized intersections, passenger boarding/alighting activities, lift use, bus load, time of day, driver experience, departure delay, travel direction, bus vehicle type, route type and weather. Some of these factors affect bus travel time between stops such as distance, number of signalized intersections, departure delay, traffic conditions and route type; some factors affect bus travel time at stops (dwell time) such as passenger boarding/alighting activities, lift use and number of bus stops; other factors affect bus travel time both between stops and at stops such as time of day, driver experience, travel direction, bus vehicle type and weather.

\subsubsection{Factors affecting bus travel time and its variability}

This section focuses on research findings regarding the impacts of factors that affect bus travel time between bus stops, followed by a discussion of factors that affect bus dwell time at bus stops.

Travel distance is one of the most important factors that are highly associated with bus travel time. However, the estimated coefficients vary significantly in the literature, the equivalent travel speeds (the reciprocal of estimated distance coefficient, e.g. 180 seconds per mile is equivalent to $20 \mathrm{mph}$ ) range between $10 \mathrm{mph}$ and $32 \mathrm{mph}$ (Abkowitz and Engelstein, 1984; Bertini and El-Geneidy, 2004; El-Geneidy and Surprenant-Legault, 
2010; El-Geneidy et al., 2011; Figliozzi and Feng, 2012; Strathman et al., 2002, 2000). This may be because traffic conditions, bus route and roadway characteristics are different among these studies and different independent variables were used in these models.

A few studies investigated the impact of signalized intersections on bus travel time. However, the average delay associated to each signalized intersection varies significantly because studies were conducted on different urban arterials with different intersection signal timing and geometric characteristics. For example, Abkowitz and Engelstein (1984), El-Geneidy et al. (2009), McKnight et al. (2004) and Albright and Figliozzi (2012a) found that each intersection adds an average of 8,26, 11 and 10 seconds to bus travel time, respectively. El-Geneidy et al. (2009) and Figliozzi and Feng (2012) also found that each stop sign adds an average of 16 and 12 seconds to bus travel time, respectively. Furthermore, Figliozzi and Feng (2012) estimated the effects of signalized intersections on bus travel time in three conditions. They found that the average additional travel time due to a bus passing through, turning left and turning right at an intersection are 5, 20 and 38 seconds, respectively. This may be because intersection signal timing characteristics are different among intersections and by bus travel direction. To the best of our knowledge, there is no study that has analyzed the effect of signalized intersections on bus travel time by incorporating intersection signal timing characteristics such as average red phase duration or red phase duration over cycle length (RC) ratio. 
The literature agrees that traffic impact is another important variable that affects bus travel time and its variability. In theory, increasing traffic flow may create additional delay to transit vehicles due to traffic congestion along the route or vehicle queuing near signalized intersections. Also, increasing traffic flow may lead to more lane changing activities that delay transit vehicles. However, most research studied the impact of traffic conditions on bus travel time using "time of day" or "travel direction" variables rather than a traffic flow variable due to the lack of traffic flow data. The effects of "time of day" and "travel direction" were found to be significant in all of the previous studies, but they are not direct measures of traffic condition. This research will utilize the archived traffic volume data to directly estimate the impact of traffic conditions on bus travel time.

The number and spacing of bus stops also affect bus travel time (route level or time point segment level) and its variability. Most studies used the actual number of stops made in a trip as a variable (Diab and El-Geneidy, 2012; Figliozzi and Feng, 2012; Slavin et al., 2013; Strathman et al., 2002, 2000; Tétreault and El-Geneidy, 2010), and they found that the additional travel time associated to each additional bus stop ranges between 5 seconds and 26 seconds. El-Geneidy et al. (2011), EI-Geneidy et al. (2009) and McKnight et al. (2004) used the number of scheduled bus stops as the variable, and they found an average of 5-13 seconds increase in travel time for each additional bus stop. The number of scheduled bus stops has a less effect on bus travel time than the number of actual bus stops because buses may skip some bus stops during a trip. El-Geneidy and SurprenantLegault (2010) also used the percentage of scheduled bus stops that were actually made 
as a variable to analyze its impact on bus travel time; this variable was found to be positive and significant.

Other variables were also found to have significant impacts on bus travel time. For example, bus departure delay was found to have negative impact on bus travel time (ElGeneidy and Surprenant-Legault, 2010; Figliozzi and Feng, 2012; Strathman et al., 2002), which indicates that buses travel faster when they are late. Diab and El-Geneidy (2012, El-Geneidy and Surprenant-Legault (2010), El-Geneidy et al. (2011), and Tétreault and El-Geneidy (2010) found that each additional onboard passenger reduces bus travel time by $0.3-2.3$ seconds. This may be because buses with more onboard passengers are usually late and thus they travel slightly faster to catch up to the schedule. Some studies found that bus vehicle type (Dueker et al., 2004; Figliozzi and Feng, 2012), bus route type (El-Geneidy et al., 2009; Strathman et al., 2002) and weather (Diab and El-Geneidy, 2012; El-Geneidy et al., 2011; Tétreault and El-Geneidy, 2010) have a significant impact on dwell time and bus travel time. For example, low floor buses save bus dwell time, feeder bus routes and express bus routes have lower travel times, and buses travel slower with increasing precipitation including snow.

Albright and Figliozzi (2012a) is the only study that has analyzed bus travel time at the stop-to-stop segment level. Bus stop location type was found to have a significant effect on bus travel time. It was found that near-side bus stops save an average of 3.7 seconds in travel time compared to far-side stops. Albright and Figliozzi (2012a) also investigated the effect of bus bay length on bus travel time. However, mixed results were found 
between two travel directions. In one direction, near-side and far-side bay lengths increased travel time and mid-block bay lengths decreased travel time. In the other direction, near-side bus bay length increased travel time, far side bus bay length decreased travel time and mid-block bus bay length was not significant.

Passenger boarding and alighting activities are important factors that affect bus dwell times at bus stops and consequently affect bus travel times. Abkowitz and Engelstein (1984) found that each passenger boarding and alighting movement increases bus travel time by 6 and 4 seconds, respectively. These estimated parameters are large compared to findings in later studies because the number of bus stops was not included in this study. After controlling for the number of bus stops, the effect of passenger boarding was found to be 3.4-3.7 seconds, and the effect of passenger alighting was found to be $0.4-1.5$ seconds (Bertini and El-Geneidy, 2004; Dueker et al., 2004; Figliozzi and Feng, 2012; Slavin et al., 2013). Dueker et al. (2004), El-Geneidy et al. (2009) and Figliozzi and Feng (2012) also tested the second order effects of passenger boarding and alighting activities. These variables were found to have negative effects on bus travel time and dwell time. The interpretation of these variables is that the incremental impact of each passenger boarding or alighting to bus travel time is lower as the number of boarding/alighting passengers increases. El-Geneidy and Surprenant-Legault (2010), El-Geneidy et al. (2011) and Strathman et al. (2002) used the total number of boarding and alighting passengers to predict bus travel time. Results showed that each additional passenger adds 1.4-3.4 seconds to travel time. The squared terms of the total number of boarding and alighting passengers were also found to be significant and negative. This indicates that removing 
some bus stops and increasing passenger activity at fewer bus stops can lead to a reduction in bus travel time increment associated with each passenger activity, and subsequently reduce total travel time (EI-Geneidy, 2005).

Other studies have used different performance measures (or dependent variables) to analyze bus travel time. These performance measures include bus travel time delay/deviation (actual travel time minus scheduled travel time) (El-Geneidy et al., 2011, 2009; Strathman et al., 1999), travel time standard deviation (Mazloumi et al., 2010) and travel time coefficient of variation (Diab and El-Geneidy, 2013; El-Geneidy et al., 2011, 2009; Tirachini, 2013). Impact factors that were significant in these models are similar to those are found in bus travel time models. A summary of independent variables that have been used in the literature is presented in Table 1-1.

In summary, there is no study that has analyzed the joint impact of signal delay, traffic conditions and bus stop location on bus stop-to-stop travel time across an intersection, by incorporating intersection signal timing parameters or directly measuring traffic volumes at each segment. This study will fill in these gaps and in order to obtain more insights into understanding and measuring the impacts of these factors on bus travel time and its variability. 
Table 1-1 Summary of Bus Travel Time Modeling Literature

\begin{tabular}{|c|c|c|c|c|c|c|c|c|c|c|c|}
\hline Literaturelindependent variables & Distance & $\begin{array}{l}\text { Ons, } \\
\text { offs }\end{array}$ & stops & Signals & $\begin{array}{l}\text { Time } \\
\text { of day }\end{array}$ & Direction & $\begin{array}{l}\text { Depart } \\
\text { delay }\end{array}$ & $\begin{array}{l}\text { Vehicle } \\
\text { type }\end{array}$ & $\begin{array}{l}\text { Route } \\
\text { type }\end{array}$ & Weather & Load \\
\hline (Abkowitz and Engelstein, 1984) & $\sqrt{ }$ & $\sqrt{ }$ & & $\sqrt{ }$ & $\sqrt{ }$ & $\sqrt{ }$ & & & & & \\
\hline (Strathman et al., 2000) & $\sqrt{ }$ & $\sqrt{ }$ & $\sqrt{ }$ & & $\sqrt{ }$ & $\sqrt{ }$ & $\sqrt{ }$ & & $\sqrt{ }$ & & \\
\hline (McKnight et al., 2004) & & $\sqrt{ }$ & $\sqrt{ }$ & $\sqrt{ }$ & & & & & & & \\
\hline (Bertini and El-Geneidy, 2004) & $\sqrt{ }$ & $\sqrt{ }$ & $\sqrt{ }$ & & & & & & & & \\
\hline (El-Geneidy et al., 2009) & $\sqrt{ }$ & $\sqrt{ }$ & $\sqrt{ }$ & $\sqrt{ }$ & $\sqrt{ }$ & $\sqrt{ }$ & & & $\sqrt{ }$ & & $\sqrt{ }$ \\
\hline (El-Geneidy et al., 2011) & $\sqrt{ }$ & $\sqrt{ }$ & $\sqrt{ }$ & & $\sqrt{ }$ & $\sqrt{ }$ & $\sqrt{ }$ & & & & $\sqrt{ }$ \\
\hline (El-Geneidy and Surprenant-Legault, 2010) & $\sqrt{ }$ & $\sqrt{ }$ & $\sqrt{ }$ & & $\sqrt{ }$ & $\sqrt{ }$ & $\sqrt{ }$ & & $\sqrt{ }$ & & \\
\hline (Tétreault and El-Geneidy, 2010) & & $\sqrt{ }$ & $\sqrt{ }$ & & $\sqrt{ }$ & $\sqrt{ }$ & $\sqrt{ }$ & & & $\sqrt{ }$ & $\sqrt{ }$ \\
\hline (Figliozzi and Feng, 2012) & $\sqrt{ }$ & $\sqrt{ }$ & $\sqrt{ }$ & $\sqrt{ }$ & $\sqrt{ }$ & & $\sqrt{ }$ & $\sqrt{ }$ & & & \\
\hline (Albright and Figliozzi, 2012b) & $\sqrt{ }$ & & & $\sqrt{ }$ & $\sqrt{ }$ & $\sqrt{ }$ & $\sqrt{ }$ & & & & \\
\hline (Diab and El-Geneidy, 2012) & & & $\sqrt{ }$ & & $\sqrt{ }$ & & $\sqrt{ }$ & $\sqrt{ }$ & $\sqrt{ }$ & $\sqrt{ }$ & $\sqrt{ }$ \\
\hline (Slavin et al., 2013) & & $\sqrt{ }$ & $\sqrt{ }$ & & $\sqrt{ }$ & $\sqrt{ }$ & & & & & \\
\hline
\end{tabular}




\subsubsection{Transit signal priority performance evaluation}

A considerable number of researchers have proposed strategies to improve transit service reliability and have evaluated their impacts on bus travel time and service reliability. These strategies include bus stop consolidation and relocation (El-Geneidy et al., 2006; Furth and Rahbee, 2000; Li and Bertini, 2009; Saka, 2001), bus rapid transit (BRT) implementation (Levinson et al., 2003), smart card payment system (Diab and ElGeneidy, 2012; Tirachini, 2013), bus holding (Abkowitz and Lepofsky, 1990; Abkowitz et al., 1986; Eberlein et al., 2001; Sun and Hickman, 2008) and expressing strategies (Eberlein et al., 1998; Fu et al., 2003; Sun and Hickman, 2005), as well as TSP implementation (Dion and Hellinga, 2002; Kimpel et al., 2005; Skabardonis, 2000). However, bus stop consolidation and relocation, BRT implementation and smart card payment system strategies are usually not easy to be implemented without a careful costbenefit analysis process. Bus holding strategies reduce some passengers' out-of-vehicle waiting time but increase on-board passengers' in-vehicle waiting time, and vice versa for bus expressing strategies. Compared to these strategies, TSP is a relatively inexpensive and easy to be implemented tool that can make transit service more reliable, faster and more cost effective (Smith et al., 2005).

TSP is the process of detecting transit vehicles approaching signalized intersections and adjusting the phasing of the signal in real time to reduce the delay experienced by the transit vehicle (Furth and Muller, 2000). The two most common TSP phases are green extension and early green (or red truncation). Both are expected to reduce bus travel time 
delay at intersections. Green extension extends a regular green phase for a certain amount of time to help transit vehicles pass through the intersection before the green signal turns to red. Early green truncates a regular red phase for a few seconds and begins the green phase early to help transit vehicles start moving early. According to Smith et al. (2005), a TSP system typically consists of three components: (1) a priority request generator on a bus that alerts the traffic control system that the bus would like to receive priority; (2) a detection system that receives the priority request and lets the traffic controller know where the bus is located; (3) priority control strategies that help the signal controller make decisions regarding whether to grant a TSP phase, which TSP phase should be granted, and when the TSP phase should start and end. There are a variety of priority control strategies that can be classified into three categories: unconditional (or passive) priority, conditional (or active) priority and real-time optimal priority. Passive priority grants a priority phase regardless of the state of the intersection or the bus. Active priority grants a priority phase only when the states of the bus and the intersection meet certain requirements. The duration of the green extension and early green phases are usually constant. Real-time optimal priority strategies make TSP decisions in real-time based on the states of the bus and the intersection, as well as the objectives of the decision makers. The objective may be to minimize the total passenger delay of an intersection (Christofa and Skabardonis, 2011; Mirchandani and Lucas, 2004), to minimize bus schedule deviations (Ma et al., 2013, 2010), or to minimize other composed performance measures (Conrad et al., 1998; Dion and Hellinga, 2002; He et al., 2011; Yagar and Han, 1994). Therefore, TSP phase start time and duration may vary from cycle to cycle. 
A good number of researchers focus on evaluating the effects of proposed TSP strategies on transit vehicles and other traffic utilizing analytic or simulation models. However, results vary significantly in the literature. Balke et al. (2000) simulated an active priority strategy at an isolated intersection with both green extension and early green phases. They found significant reductions in bus travel time at different traffic levels with minor increases in total intersection delay under moderate traffic levels. Furth and Muller (2000) evaluated the performance of passive and active TSP systems in a corridor using simulation. Results showed that both passive and active priority significantly improved bus schedule adherence. However, active priority has almost no impact on traffic delay and passive priority significantly increased traffic delay. Skabardonis (2000) proposed both passive and active priority strategies and evaluated them on a corridor with 21 coordinated intersections through simulation. This study showed that TSP strategies provide modest improvement for the buses without adverse effects on automobile traffic. Dion et al. (2004) evaluated the performance of several active priority strategies using simulation models on an arterial corridor. They found that buses would typically benefit from TSP but at the expense of the overall traffic. However, when traffic flow on the side street is low, the overall negative impacts can be negligible. Byrne et al. (2005) evaluated the effectiveness of a conditional TSP system at a single intersection using a simulation model. Results showed that the TSP implementation yields an $11 \%$ reduction in bus travel time for the far-side stop configuration and a $6 \%$ increase in bus travel time for the near-side stop consolidation. Some studies claimed that TSP is more efficient at far-side bus stops because there is less uncertainty in predicting the arrival time of a bus at an 
intersection (Chada and Newland, 2002). Therefore, bus arrival time prediction, especially upstream of intersection, is important to the effectiveness of TSP.

Unlike most of the previous studies that use simulation models to study the effect of TSP system on buses and other traffic, Lin (2002) used analytical models to quantify the transit vehicle delay reduction due to signal priority. He found that delay reduction is especially small for buses traveling on the major street of an arterial, and buses from minor streets are expected to receive higher delay reduction. In summary, most of the proposed TSP control strategies were evaluated under idealistic assumptions and were based on either analytic or simulation models without ground tests on real world data. Also, mixed results were found in the literature regarding the impact of TSP system on buses and other vehicles, this may be because TSP performance is a function of many factors including intersection geometry, signal timing, traffic demand, TSP control strategies and parameters, transit vehicle headways, reliability of detection system and the TSP request generating system (Abdy and Hellinga, 2011).

There are also a few studies that evaluated the effects of TSP on buses and other vehicles by using real world collected data. However, results are not consistent in the literature. For example, Hunter-Zaworski et al. (1995) collected travel time data for buses and other vehicles at four intersections on Powell Blvd. in Portland, Oregon, before and after the implementation of an active TSP system. They found that after TSP implementation, bus travel time decreased during peak hours but increased during off-peak hours, and intersection total person delay had mixed changes at different times of day. Subsequently, 
Koonce et al. (2002) evaluated the impact of a TSP system on bus travel time on another corridor (Barbur Blvd.) in Portland, Oregon. Results showed that bus travel time decreased 0.4-3.2 minutes and travel time variability decreased 2.2-19.2\% during different times of day and travel directions. However, no difference was found in bus travel time savings between buses that were late and those that were not late. Kimpel et al. (2005) evaluated changes in bus running times, on-time performance, and excess passenger waiting times following TSP implementation on several corridors in Portland, Oregon. Results showed that the benefits of TSP are not consistent across routes and time periods, nor are they consistent across various performance measures. Slavin et al. (2013) evaluated the effect of TSP on bus travel time using regression models. Results showed that the TSP system significantly reduced bus travel time over the study corridor for buses that requested TSP after controlling for other factors. Albright and Figliozzi (2012b) also utilized regression models to study the effect of TSP on bus headways on the same corridor. Results showed that a bus that requested signal priority significantly shortened the headway to its preceding bus and increased the headway to its following bus. Another study by Albright and Figliozzi (2012c) was conducted on the same corridor. This study evaluated the effect of TSP on bus schedule recovery (bus schedule delay before and after an intersection) at several intersections along this corridor. Results showed that the effect of TSP on bus schedule delay varies across intersections. This study found that late bus schedule recovery is greater at intersections with less demand on the minor crossing streets. Diab and El-Geneidy (2012), Diab and El-Geneidy (2013) utilized regression models to study the impact of a TSP system on bus travel time and its variability on two bus routes in Montreal, Canada. Active TSP systems were implemented in these two 
corridors. Results indicated that bus travel times for the two bus routes significantly decreased after the implementation of a TSP system, and that TSP equipped buses have shorter travel times than those buses that were not equipped with TSP. However, the bus travel time coefficient of variation increased after the implementation of the TSP system and for TSP equipped buses.

In summary, previous studies only analyzed the impacts of TSP systems on bus travel time savings, on-time performance, headways, and the delay and time savings for other vehicles; no one has evaluated the effectiveness of TSP phases or the relationships between TSP requests and TSP phases. For example, it is possible that 10 buses requested signal priority at an intersection, 20 TSP phases were granted in the same time period, but only 5 buses benefited from these TSP phases due to uncertainty of the traffic conditions or TSP systems reliability issues. There is no study that has accessed TSP phase log data, or integrated TSP phase log data with bus AVL/APC data to evaluate the TSP system performance. Therefore, this study will fill in this gap and evaluate TSP system performance by integrating unique data sources and proposing novel performance measures.

\subsection{Objectives}

The objectives of this study are to: 
1. Develop an algorithm to integrate bus AVL/APC data, SCATS signal phase log data and intersection traffic count data into one synthesized database for bus travel time regression analysis and TSP performance evaluation;

2. Estimate the joint impact of traffic signal control, traffic conditions and bus stop location on bus travel time at the stop-to-stop segment level by estimating regression models; and

3. Evaluate TSP system performance by evaluating TSP phase effectiveness (percent of TSP phases that benefited buses), bus and passenger time savings and other vehicles time savings and delay, for green extension and early green phases separately.

\subsection{Dissertation Organization}

This study consists of five chapters. The first chapter has introduced the motivation, literature review and objectives of this study. Chapter Two describes the data sources used in this study and how they are integrated. Chapter Three presents several bus stopto-stop travel time models for far-side segments only, for all stop-to-stop segments together, and for each individual stop-to-stop segment. Chapter Four evaluates the TSP system performance based on the integration of bus AVL/APC data and SCATS phase $\log$ data. Chapter Five summarizes the findings of this study and provides implications and recommendations for cities and transit agencies. Future research directions are also discussed. 


\section{DATA AND METHODOLOGY}

\subsection{Study Corridor Description}

Powell Boulevard is a major commuter arterial located in Portland, Oregon. Bus route 9 is the primary bus route operated along this corridor. Route 9 runs east-west with an average headway of 15 minutes during midday and an average headway of 6-7 minutes during the morning and evening peak periods.

The study corridor, signalized intersections and bus stops are shown in Figure 2-1. The study corridor is a 4-mile long urban arterial corridor with two lanes in each direction, downtown Portland is located to the west of the figure. Westbound (WB) peak traffic volume takes place in the morning rush hour (towards downtown Portland); eastbound (EB) peak traffic volume takes place in the evening rush hour (away from downtown Portland). The Sydney Coordinated Adaptive Traffic System (SCATS) is implemented in 12 signalized intersections between Milwaukie Ave. and $72^{\text {nd }}$ Ave. Because cycle length, green phase and red phase at each intersection vary over time in the SCATS system, the median cycle length, red phase and green phase are shown in Figure 2-2. The red phase duration for the EB and WB directions include both major street left turn protected phase duration and cross street green phase duration. Most of the intersections have similar cycle lengths, 120 seconds on average. Red phase duration varies significantly across intersections; buses may experience longer delays at some major intersections such as 
Milwaukie (MKE), $39^{\text {th }}, 50^{\text {th }}$ and $52^{\text {nd }}$ Ave. Transit signal priority (TSP) is programmed to respond to bus priority requests from both the EB and WB directions at each of the 12 intersections.

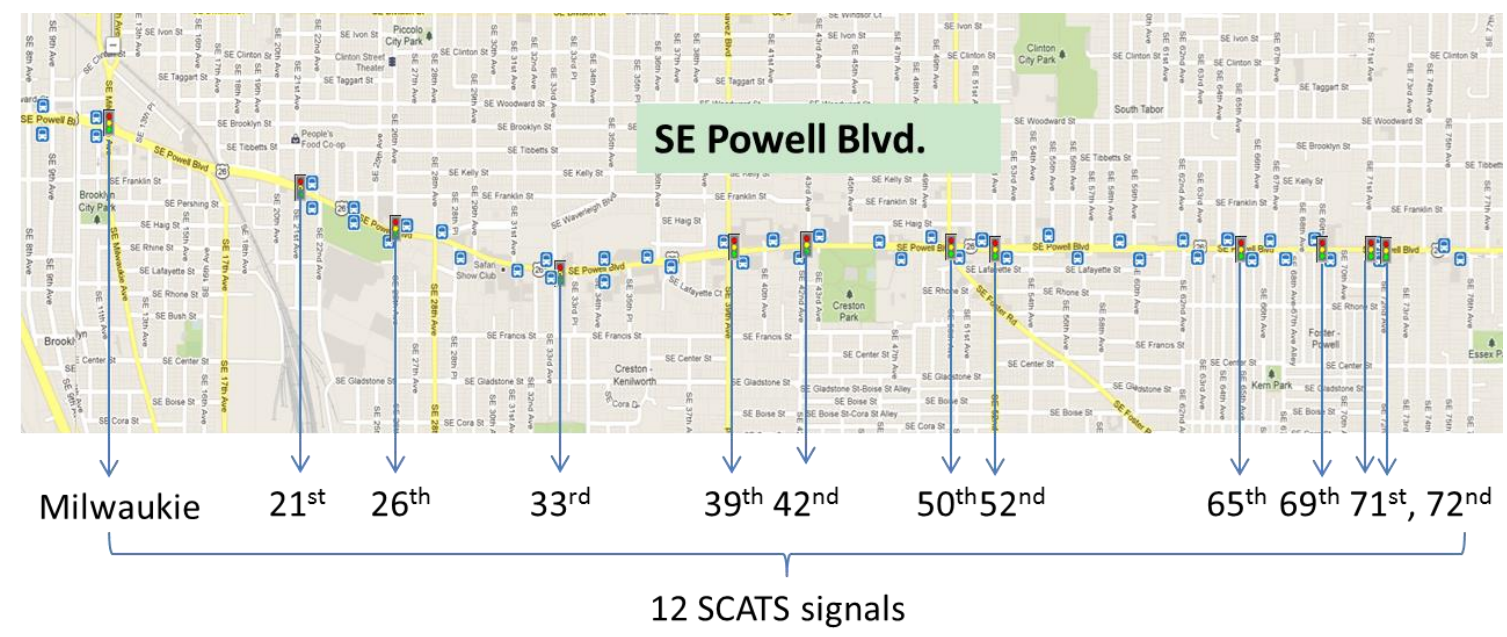

Figure 2-1 Study corridor overview

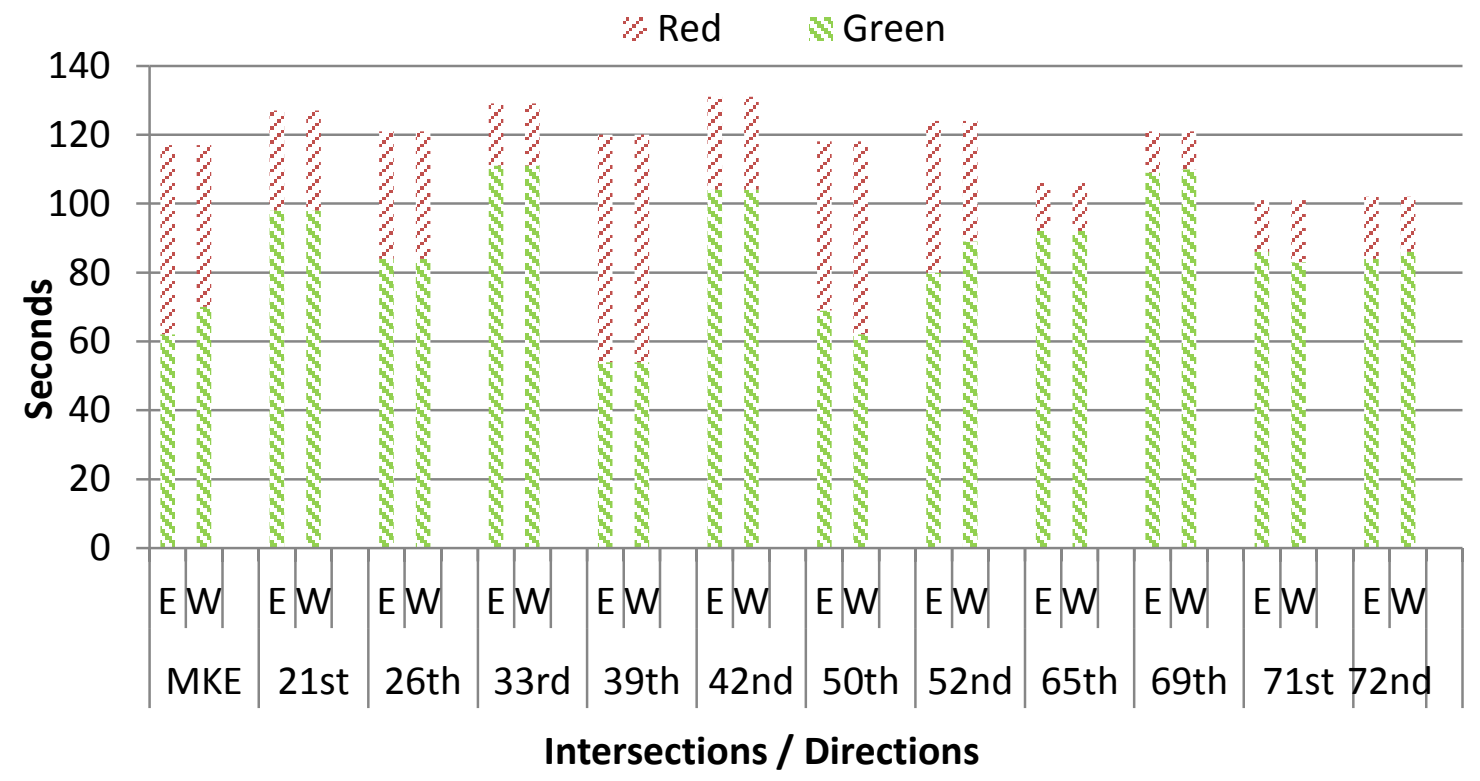

Figure 2-2 Median cycle length, red phase and green phase durations by direction 
$33^{\text {rd }} \mathrm{EB}$

$26^{\text {th }}$ WB

$42^{\text {nd }} W B$

$26^{\text {th }} \mathrm{EB}$

$72^{\text {nd }} E B$

$42^{\text {nd }} E B$

$65^{\text {th }}$ WB

$52^{\text {nd }} E B$

$39^{\text {th }}$ WB

$69^{\text {th }} E B$

$71^{\text {st }} \mathrm{EB}$

$65^{\text {th }} \mathrm{EB}$

$50^{\text {th }}$ WB

$33^{\text {rd }}$ WB

$52^{\text {nd }} W B$

$39^{\text {th }} \mathrm{EB}$

$50^{\text {th }} \mathrm{EB}$

$72^{\text {nd }}$ WB

$71^{\text {st }} \& 69^{\text {th }} W B$ Milwaukie \& $21^{\text {st }} \mathrm{EB}$

$21^{\text {st }} \&$ Milwaukie WB
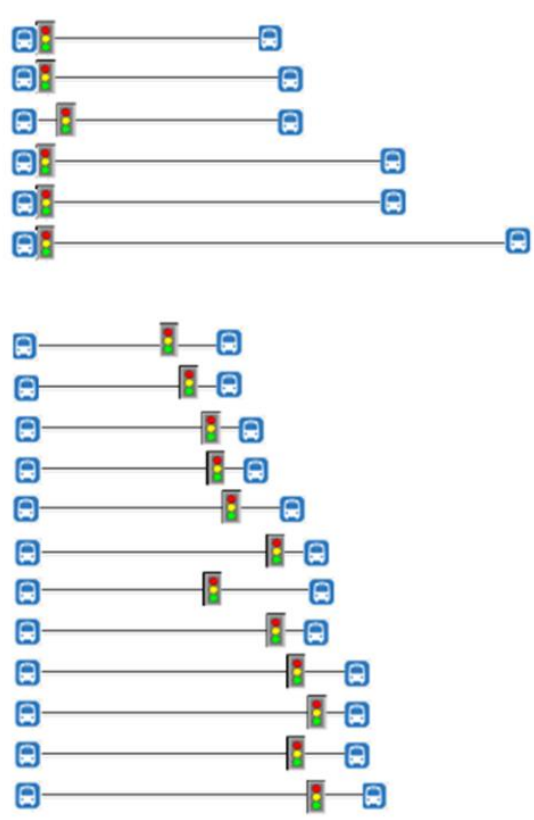

Far-side segments
Near-side segments

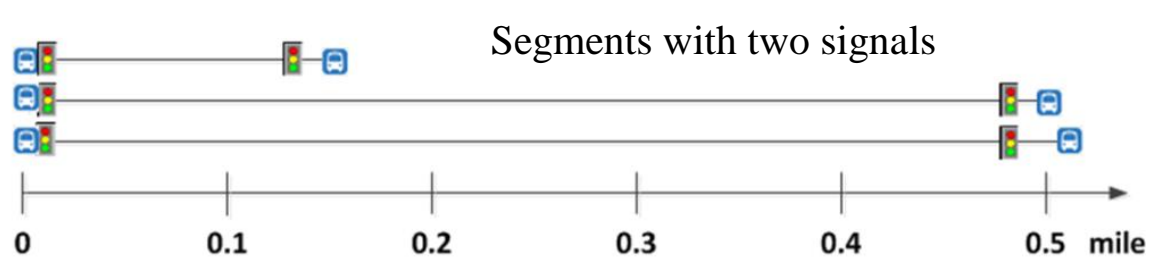

Figure 2-3 Bus stop-to-stop segments that include SCATS signals

There are 22 bus stops and 21 bus stop-to-stop segments in each direction between Milwaukie and $72^{\text {nd }}$ Ave. These bus stop-to-stop segments are classified into four categories: near-side segment, far-side segment, segment with two signals and segment without a signal. Near-side segment means the departure stop of the stop-to-stop segment is a near-side stop; far-side segment means the arrival stop of the stop-to-stop segment is a far-side stop. In the intersections between Milwaukie and $72^{\text {nd }}$ Ave., there are 6 nearside segments, 12 far-side segments and 3 segments with two signals. Figure 2-3 shows the segment lengths and the signal position in each of the 21 stop-to-stop segments that have at least one signal. " $33^{\text {rd }}$ EB" segment indicates the stop-to-stop segment that cross the intersection of $33^{\text {rd }}$ Ave. in the eastbound travel direction. Most of these segments are 
less than 0.2 miles long. Near-side bus stops are much closer to intersections than far-side stops. In this study, only 12 far-side segments and 6 near-side segments are chosen for bus travel time modeling analysis and TSP performance evaluation, because it is difficult to integrate the signal phase data with bus AVL/APC data when there are two signals in one stop-to-stop segment.

\subsection{Data Description}

Three archived databases were used for this corridor to conduct the analyses: bus AVL/APC data, SCATS signal phase log data and traffic count data. In the bus AVL/APC data, every time a bus makes a stop, the arrival time, departure time and schedule time are recorded; other information such as stop location, vehicle information, passenger activities, onboard passengers and dwell time are also recorded. According to the TriMet Bus Dispatching System (BDS) AVL/APC data dictionary, each bus stop in the TriMet system is referenced by a 50-foot stop circle in the agency's Geographic Information System (GIS). Arrival time refers to the time that a bus first enters the 50foot stop circle except when a door opening occurs. If a door opening occurs within the stop circle, then arrival time is overwritten with the time of the door opening. Departure time refers to the time when a bus leaves the 50-foot stop circle. However, some nearside bus stops are close enough to the intersection stop bar (less than 50 feet) that signal delay for buses at near-side stops is not included in the time interval between the departure time from this near-side stop and the arrival time in the next bus stop. This issue leads to different modeling strategies between near-side and far-side bus stops to 
study the impact of signal delay on bus travel time reliability. Schedule time refers to the scheduled departure time for a bus stop (TriMet, 2013).

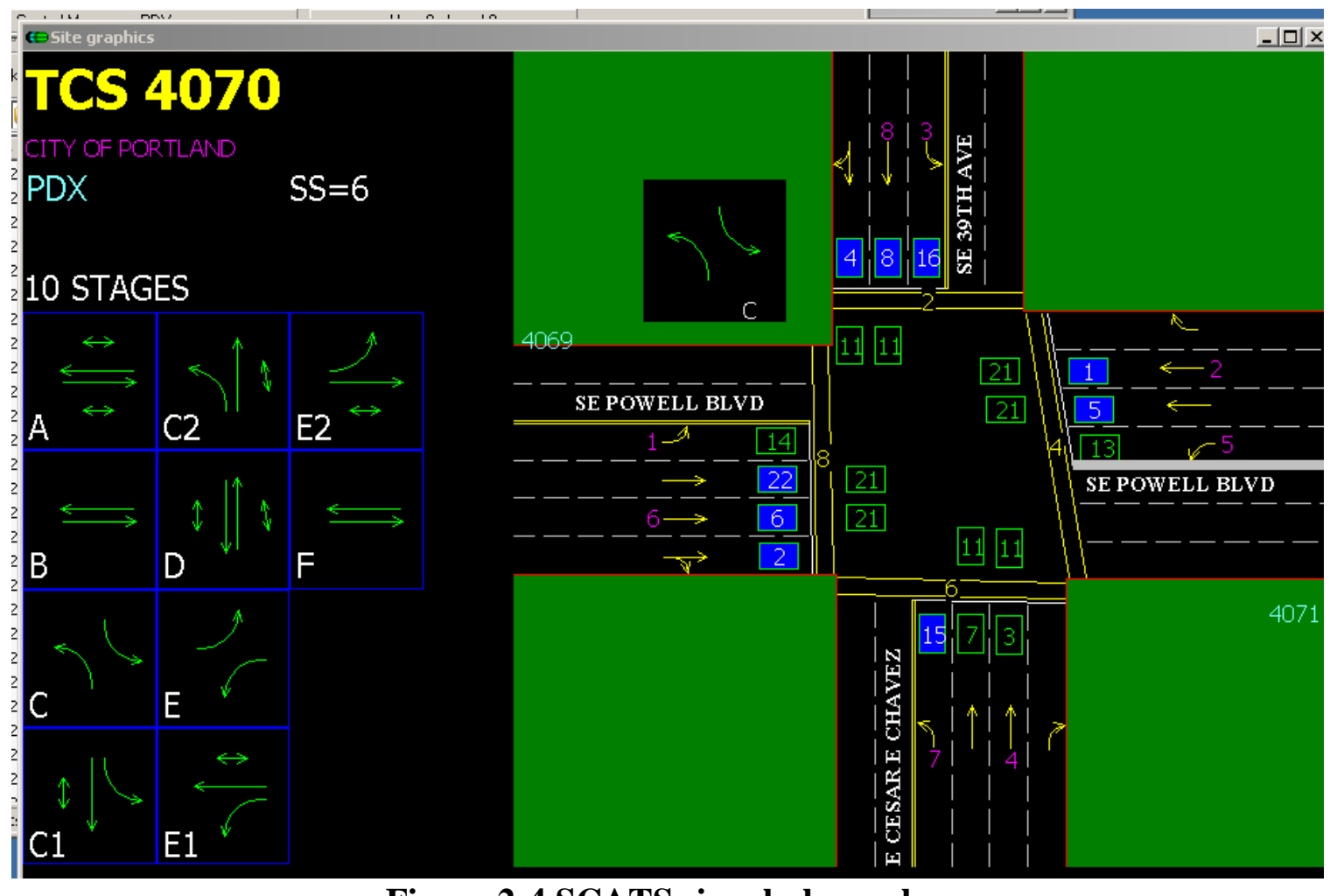

Figure 2-4 SCATS signal phase plan

(Source: Portland Bureau of Transportation SCATS system, City of Portland)

The SCATS phase log data provides the start time and end time of each phase (including TSP phase) in a cycle. For example, Figure 2-4 and Figure 2-5 show the SCATS signal phase plan and the disaggregated (original) SCATS phase log data for the intersection of $39^{\text {th }}$ Ave. and Powell Blvd, respectively. Phases are granted in the order of "A", "B", "C", "D", "E" and "F". Phases "C1" and "C2" are extension phases of phase "C"; phases "E1" and "E2" are extension phases of phase "E"; both of them are mutually exclusive. Phase " $\mathrm{A}$ " is the regular green phase for the EB and WB through movement. Phase " $\mathrm{B}$ " and 
phase "F" are the green extension (GE) phase and early green (EG) phase. In this study, we are only interested in the regular green phase, red phase, green extension phase and early green phase for the EB and WB through movement. Therefore, phases "C", "C1", "C2", "D", "E" and "E1" are aggregated into one red phase for the EB through movement; phases "C1", "C2", "D", "E" and "E2" are aggregated into one red phase for the WB through movement. Similar phase aggregation is applied to other intersections along this corridor, although the phase order varies significantly across intersections.

\begin{tabular}{|c|c|c|c|c|c|c|c|c|}
\hline 20 & 13-03-04_39th_ & Oowell_SCATSphase. & xt - Notepad & & & 口 & 回 & $?$ \\
\hline File & Edit Format & View Help & & & & & & \\
\hline Date & & start Time & End Time & Duration & Phase & Gap & Error & $\Delta$ \\
\hline Mon & $4-\mathrm{Mar}-2013$ & $07: 23: 29$ & $07: 24: 33$ & 64 & & Gap & EI T & 2 \\
\hline Mon & $4-\mathrm{Mar}-2013$ & $07: 24: 33$ & $07: 24: 53$ & 20 & $\hat{c}$ & & & \\
\hline Mon & 4-Mar-2013 & $07: 24: 53$ & $07: 25: 40$ & 47 & D & Yes & & \\
\hline Mon & 4-Mar-2013 & $07: 25: 40$ & $07: 26: 43$ & 63 & A & & & \\
\hline Mon & $4-\mathrm{Mar}-2013$ & $07: 26: 43$ & $07: 26: 50$ & 7 & B & & & \\
\hline Mon & $4-M a r-2013$ & $07: 26: 50$ & $07: 27: 07$ & 17 & $c$ & & & \\
\hline Mon & $4-M a r-2013$ & $07: 27: 07$ & $07: 27: 42$ & 35 & D & & & \\
\hline Mon & $4-\mathrm{Mar}-2013$ & $07: 27: 42$ & $07: 27: 52$ & 10 & $\mathrm{E}$ & & & \\
\hline Mon & $4-\mathrm{Mar}-2013$ & $07: 27: 52$ & $07: 28: 02$ & 10 & $\mathrm{~F}$ & & & \\
\hline Mon & $4-\mathrm{Mar}-2013$ & $07: 28: 02$ & $07: 28: 53$ & 51 & A & & & \\
\hline Mon & 4-Mar-2013 & $07: 28: 53$ & $07: 29: 17$ & 24 & c & & & \\
\hline Mon & $4-M a r-2013$ & $07: 29: 17$ & $07: 29: 52$ & 35 & D & & & \\
\hline Mon & $4-\mathrm{Mar}-2013$ & $07: 29: 52$ & $07: 30: 07$ & 15 & $\mathrm{E}$ & & & \\
\hline Mon & $4-\mathrm{Mar}-2013$ & $07: 30: 07$ & $07: 31: 04$ & 57 & A & & & \\
\hline Mon & $4-\mathrm{Mar}-2013$ & $07: 31: 04$ & $07: 31: 24$ & 20 & c & & & \\
\hline Mon & $4-M a r-2013$ & $07: 31: 24$ & $07: 31: 48$ & 24 & D & Yes & & \\
\hline Mon & $4-M a r-2013$ & $07: 31: 48$ & $07: 32: 14$ & 26 & $\mathrm{E}$ & Yes & & \\
\hline Mon & $4-\mathrm{Mar}-2013$ & $07: 32: 14$ & $07: 33: 13$ & 59 & A & & & \\
\hline Mon & $4-\mathrm{Mar}-2013$ & $07: 33: 13$ & $07: 33: 20$ & 7 & $B$ & & & \\
\hline Mon & $4-M a r-2013$ & $07: 33: 20$ & $07: 33: 33$ & 13 & $c$ & & & \\
\hline Mon & $4-M a r-2013$ & $07: 33: 33$ & $07: 34: 08$ & 35 & D & & & \\
\hline Mon & 4-Mar-2013 & $07: 34: 08$ & $07: 34: 18$ & 10 & $\mathrm{E}$ & & & \\
\hline Mon & 4-Mar-2013 & $07: 34: 18$ & $07: 34: 28$ & 10 & $\mathrm{~F}$ & & & \\
\hline Mon & $4-\mathrm{Mar}-2013$ & $07: 34: 28$ & $07: 35: 23$ & 55 & A & & & \\
\hline Mon & $4-M a r-2013$ & $07: 35: 23$ & $07: 35: 30$ & 7 & $B$ & & & \\
\hline Mon & $4-M a r-2013$ & $07: 35: 30$ & $07: 35: 39$ & 9 & $c$ & & & \\
\hline Mon & $4-\mathrm{Mar}-2013$ & $07: 35: 39$ & $07: 36: 14$ & 35 & D & & & \\
\hline Mon & 4-Mar-2013 & $07: 36: 14$ & $07: 36: 29$ & 15 & $\mathrm{E}$ & & & \\
\hline Mon & $4-M a r-2013$ & $07: 36: 29$ & $07: 37: 33$ & 64 & A & & & \\
\hline Mon & $4-\mathrm{Mar}-2013$ & $07: 37: 33$ & $07: 37: 46$ & 13 & C & & & \\
\hline Mon & $4-M a r-2013$ & $07: 37: 46$ & $07: 38: 21$ & 35 & D & & & \\
\hline Mon & $4-\mathrm{Mar}-2013$ & $07: 38: 21$ & $07: 38: 36$ & 15 & $\mathrm{E}$ & & & \\
\hline Mon & $4-\mathrm{Mar}-2013$ & $07: 38: 36$ & $07: 39: 44$ & 68 & A & & & \\
\hline Mon & $4-\mathrm{Mar}-2013$ & $07: 39: 44$ & $07: 40: 00$ & 16 & c & & & \\
\hline Mon & $4-\mathrm{Mar}-2013$ & $07: 40: 00$ & $07: 40: 35$ & 35 & D & & & \\
\hline Mon & $4-\mathrm{Mar}-2013$ & $07: 40: 35$ & $07: 40: 50$ & 15 & $\bar{E}$ & & & \\
\hline Mon & $4-\mathrm{Mar}-2013$ & $07: 40: 50$ & $07: 41: 53$ & 63 & A & & & - \\
\hline 1 & & & & & & & 1 & \\
\hline
\end{tabular}

Figure 2-5 SCATS phase log data example

(Source: Portland Bureau of Transportation SCATS system, City of Portland) 
SCATS traffic count data offers the 15-minute interval traffic count data for each detector at the intersection. For example, Figure 2-6 shows the vehicle count data for each detector in each 15 minutes interval at the intersection of $39^{\text {th }}$ Ave. and Powell Blvd. The detector numbers are the same as those shown in Figure 2-4. There are ten movement directions at this intersection and each movement direction refers to one or two loop detectors. For example, EBTH (eastbound through) has two detectors 6 and 22, EBTHRT (eastbound through-right) has one detector 2. The number of vehicles that passed each detector in each 15-minute interval is recorded. For example, between 00:00 and 00:15, there are 20 vehicles that passed detector 6 and there are 16 vehicles that passed detector 22, the total number of vehicles between 00:00 and 00:15 is 36 for the EBTH movement. This study focuses on the through movement vehicles; therefore, the 15-minute interval traffic count from EBTH movement detectors (6 and 22) and EBTHRT movement detector (2) are aggregated into an EBTH movement traffic count. Then, the 15-minute interval traffic count is converted to vehicles per hour. For example, between 00:00 and 00:15 am, the aggregated EBTH volume for all lanes is $(20+16+6) * 4=168$ vehicles per hour. This traffic count aggregation is also applied to other intersections, although intersection detector configurations are different.

Two months of data (March and May 2013) from the three databases were used for this study because TSP system was turned off in April 2013 for system update. Therefore, the TSP system was working along this corridor in March 2013, but not in May 2013. The numbers of effective weekdays collected from March and May 2013 are 20 and 18 days, respectively. All of the three databases from the two months will be used in the bus travel 
time modeling analysis to examine whether bus travel time is significantly different before and after the time when TSP was working. Then, the bus AVL/APC data and the SCATS phase log data from March 2013 will be used to evaluate the TSP performance.

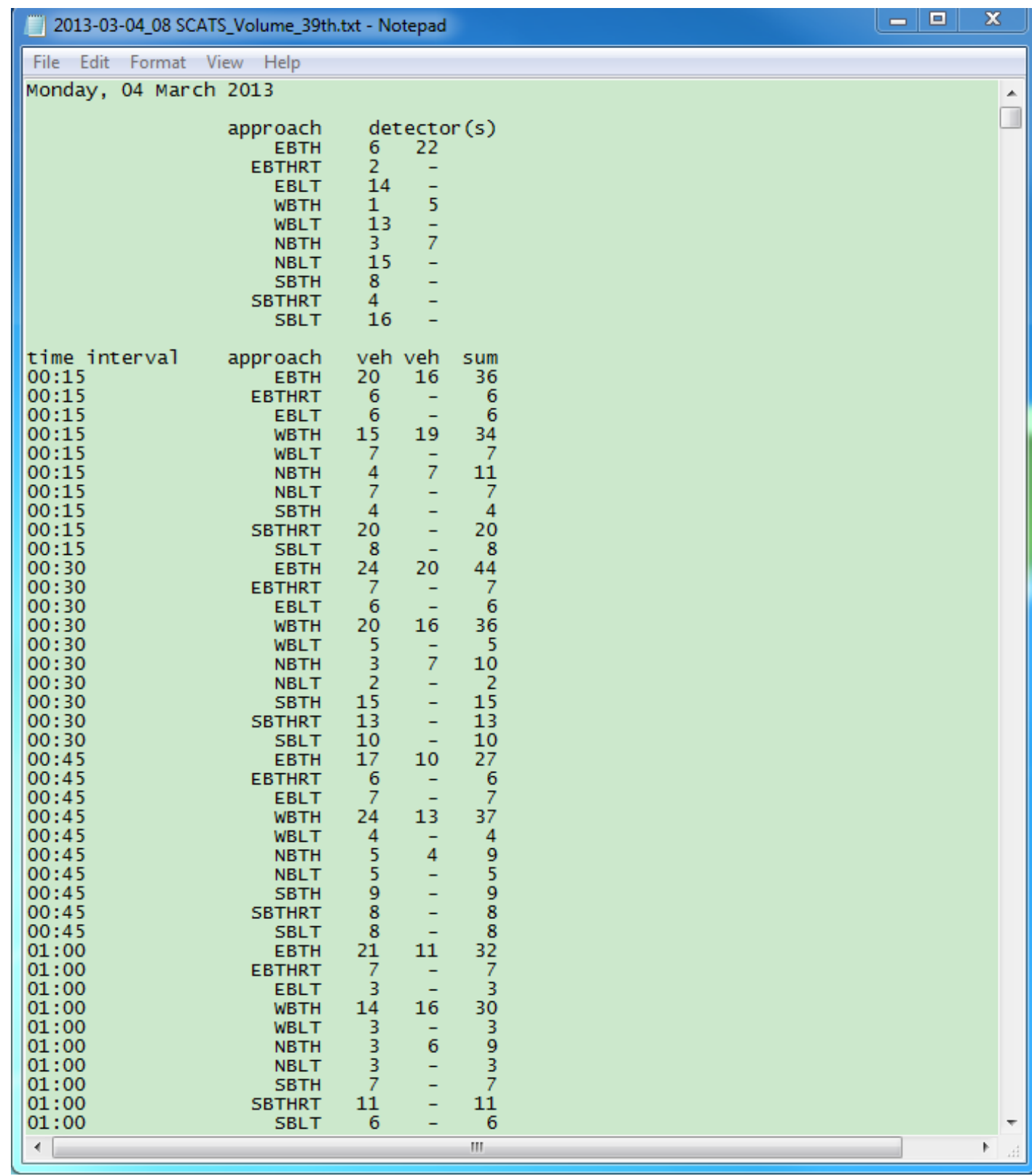

Figure 2-6 SCATS traffic count data example

(Source: Portland Bureau of Transportation SCATS system, City of Portland) 


\subsection{Research Methodology}

The methodology of this study includes three steps. The first step is to develop an algorithm to integrate the three databases and compute useful bus stop-to-stop trip attributes for later analysis. The second step is to apply multiple linear regression techniques to analyze bus stop-to-stop travel time. The last step is to propose new and useful performance measures to evaluate TSP system performance.

Data integration is important because it provides all the required information for the bus travel time modeling and TSP performance analyses. It is also a challenging step because the bus AVL/APC data and SCATS data are collected in different spatial dimensions. Bus AVL/APC data are collected at bus stops while SCATS data are collected at intersections. Bus trajectory information is unknown between bus stops. However, bus stop-to-stop travel time modeling and TSP performance analyses at the intersection level require bus arrival time information at intersections. Therefore, this study estimated bus trajectories based on empirical bus travel speed distribution in order to integrate the bus AVL/APC data and the SCATS phase log data. An integrated bus stop-to-stop trip database is created that includes three sets of attributes: bus stop activity attributes, stopto-stop segment attributes and signal phase attributes.

Ordinary least square (OLS) multiple linear regression models are developed to investigate the joint impact of traffic signal delay, traffic conditions and bus stop location on bus stop-to-stop travel time at the intersection level. Multiple regression models are 
estimated for far-side stop-to-stop segments only, all stop-to-stop segments together and individual stop-to-stop segments. For far-side stop-to-stop segments, two multiple regression models are estimated using the departure-to-arrival time and arrival-to-arrival time as the dependent variables. The departure-to-arrival time model is estimated to study the impacts of signal delay and traffic conditions on bus travel time excluding bus stop activity variability. The arrival-to-arrival time model is estimated to test the robustness of the estimated coefficients. The arrival-to-arrival time model is then estimated for all stopto-stop segments to compare the different impacts of signal delay and traffic conditions between near-side and far-side stop-to-stop segments. Furthermore, multiple regression models are also estimated for each individual segment to investigate different impacts of signal delay and traffic conditions on bus travel time, and to identify potential problems for certain intersections.

TSP performance analyses are also based on the integrated bus stop-to-stop trip database. New and useful performance measures are proposed to evaluate TSP effectiveness and benefits for buses that requested TSP. This section evaluates TSP system performance from three perspectives: the relationships between TSP requests and TSP phases, and the effectiveness and benefits of TSP requests and TSP phases. The analyses of the relationships between TSP requests and TSP phases investigate how timely TSP phases were granted in respond to TSP requests. The analyses of the effectiveness of TSP requests and TSP phases examine the percentages of TSP phases that were granted early, on-time and late. The analyses of the benefits of TSP requests and TSP phases evaluate the expected time savings for the bus that requested TSP and for the onboard passengers, 
as well as the time savings and delay for automobile traffic on the major street and the side street.

\subsection{Data Integration}

An integrated database is critical for advanced analysis of travel time regression analysis and TSP performance evaluation. Figure 2-7 shows the database integration scheme used in this study. The integrated bus stop-to-stop trip database requires information from three separate databases: bus AVL/APC data, SCATS signal phase log data and SCATS traffic count databases. A bus stop-to-stop trip is selected as the basic unit to integrate the three databases. Each bus stop-to-stop trip contains three sets of attributes: bus stop activity attributes, stop-to-stop segment attributes and signal phase attributes as shown in Table 2-1. Bus stop-to-stop trip attributes describe bus trip characteristics including constants that describe segment geometry, variables that are directly recorded by the three databases and estimated variables that are computed based on the three databases. Bus stop activity attributes are recorded variables that describe bus stop-to-stop trip characteristics at the departure and arrival bus stops of a stop-to-stop segment. Stop-tostop segment attributes include segment stationary characteristics (constants that do not vary by bus trip) and variable trip characteristics that are recorded by the bus AVL/APC data and intersection vehicle count data. Signal phase attributes are estimated variables that describe bus stop-to-stop trip characteristics related to signal phases. A new database is created to include these attributes for each bus stop-to-stop trip. This database contains 
all the necessary information required for the bus travel time regression analysis and TSP performance evaluation.

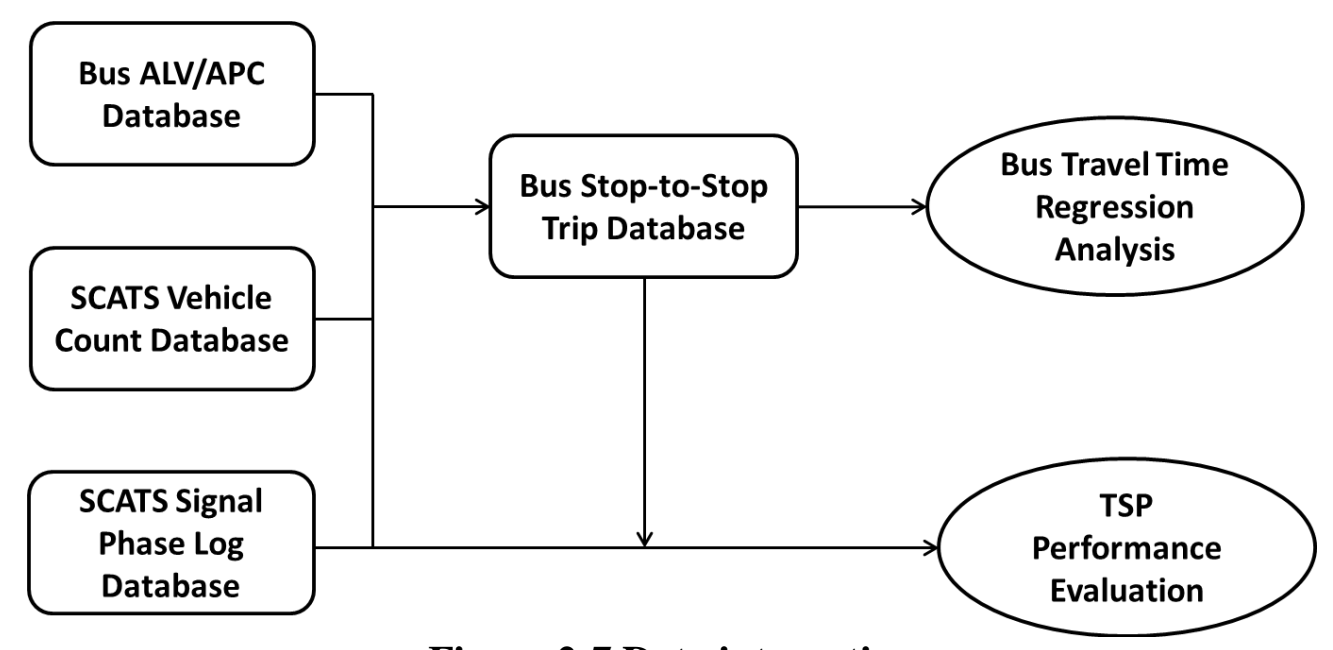

Figure 2-7 Data integration

Table 2-1 Bus stop-to-stop trip attributes

\begin{tabular}{lll}
\hline $\begin{array}{l}\text { Bus stop activity } \\
\text { attributes (recorded) }\end{array}$ & $\begin{array}{l}\text { Stop-to-stop segment } \\
\text { attributes (recorded) }\end{array}$ & $\begin{array}{l}\text { Signal phase attributes } \\
\text { (estimated) }\end{array}$ \\
\hline $\begin{array}{l}\text { Departure/arrival stop: } \\
\text { - Actual arrival time }\end{array}$ & $\begin{array}{l}\text { Constants: } \\
\text { - Stop-to-stop distance }\end{array}$ & $\begin{array}{l}\text { Estimated } \\
\text { - Red }\end{array}$ \\
- Actual departure time & - Upstream distance & - Probability of arriving in: \\
- Boarding passengers & - Downstream distance & $\circ$ Green \\
- Alighting passengers & Variables: & $\circ$ Red \\
- Number of lift use & - Departure-to-arrival time & $\circ$ Green extension \\
- TSP request (estimated) & - Arrival-to-arrival time & - Expected signal delay \\
& - Traffic volume & - Expected time savings \\
\hline
\end{tabular}

\subsubsection{Bus stop activity attributes and stop-to-stop segment attributes}

Most of the bus stop activity attributes can be directly read from the bus AVL/APC data except for TSP request, which is estimated based on the actual and scheduled departure 
times. TSP request equals one if the actual departure time is more than 30 seconds late than the scheduled departure time; otherwise, it equals zero. The constant stop-to-stop segment attributes are measured on Google Maps. Upstream distance is the distance from the upstream departure stop to the intersection stop bar and downstream distance is the distance from the intersection stop bar to the downstream arrival stop. Departure-toarrival time (or arrival-to-arrival time) is the time interval between departure from (or arrival to) the upstream departure stop and arrival at the downstream arrival stop. Traffic volume for each bus stop-to-stop trip is the 15-minute traffic flow for the bus travel direction when the trip is made. In signal phase attributes, the red attribute is a binary variable that indicates whether a bus trip encountered a red signal at the intersection. Because bus AVL/APC data only provide the bus departure time and arrival time at bus stops, the actual bus arrival time at the intersection is not known for certain. Therefore, probabilities of bus arrival time at the intersection during each phase are estimated based on an algorithm that will be explained in the following section. Based on the estimated probabilities of arriving at the intersection in each phase and the phase duration information, the expected signal delay due to a red signal and the expected time savings due to a TSP phase can be estimated. The signal phase attributes will be used for bus travel time modeling and TSP performance analyses. They are estimated based on the integration of the bus AVL/APC data and the SCATS phase log data.

\subsubsection{Signal phase attributes}


Before integrating the two data sources, it is important to compare whether the time clock systems are synchronized between the two data sources. Two two-hour videos were collected on February 26 and May 1, 2013 at the intersection of $26^{\text {th }}$ Ave. and Powell Blvd., and the videos were used as the reference clock system to compare the time clocks between the two data sources. The videos cover all four approaches of the intersection with both the bus stops and the signal heads clearly visible. Therefore, both the bus departure time and arrival time and the signal phase start time and end time recorded from the videos are in the same clock system. By comparing each pair of signal phase start time and end time records from the video and from the SCATS system, an offset between the two systems can be found. Ten bus trips were collected on each day. Results show that the video clock system is perfectly synchronized with the SCATS clock system, which means the offset between the two systems is zero. However, the bus AVL/APC data clock system is not synchronized with the SCATS clock system or the video clock system. In addition, clock systems are not synchronized between buses. In other words, the clock system on each bus has a different offset with the SCATS clock system. By comparing the bus arrival time and departure time from the video and from the bus AVL/APC data, we found that bus clock systems are 3-9 seconds later than the SCATS system. Therefore, an average offset of 5 seconds will be used when comparing the bus AVL/APC data with the signal phase start time and end time data.

Once the clock systems between the bus and the SCATS data are synchronized, algorithms can be developed to integrate the two data sources and calculate signal phase attributes. Define $\boldsymbol{I}$ as the set of bus trips for a stop-to-stop segment, and $i$ as the index for 
the $i$ th bus trip, so $i \in I$. Define $\boldsymbol{J}$ as the set of signal phase cycles for the intersection in the bus stop-to-stop segment; each cycle is defined as the time interval between two consecutive red phase start times. Because bus stop activity attributes can be directly read from the bus AVL/APC data and stop-to-stop segment attributes can be easily measured or calculated based on the bus AVL/APC data and SCATS traffic count data, these attributes and the SCATS phase log data will be used as input data to estimate the signal phase attributes.

\section{Input data}

$d_{1}, d_{2}:$ upstream distance and downstream distance as defined in Table 2-1;

$d t_{i}, a t_{i}$ : departure time from the departure stop and arrival time at the arrival stop for bus

trip $i$ (for near-side segments, $d t_{i}$ is the arrival time at the departure stop);

$\operatorname{load}_{i}$ : number of onboard passengers for bus trip $i$;

$R_{j}^{S}, R_{j}^{e}:$ red phase start time and end time for cycle $j$

$G E_{j}^{S}, G E_{j}^{e}:$ green extension phase start time and end time for cycle $j$;

$E G_{j}^{S}, E G_{j}^{e}$ : early green phase start time and end time for cycle $j$.

\section{Output signal phase attributes:}

$\operatorname{Red}_{i}$ : binary variable, it is 1 if a bus trip $i$ experienced a red signal delay at the intersection and 0 otherwise;

Prob_ $R_{i}$ : probability of arriving at the intersection during a red phase for bus trip $i$;

Prob_G $G_{i}$ : probability of arriving at the intersection during a green phase for bus trip $i$; 
Prob_GE $E_{i}$ : probability of arriving at the intersection during a green extension phase for bus trip $i$

Prob_EG $G_{i}$ : probability of arriving at the intersection during an early green phase for bus trip $i$

Exp_Delay $:$ expected red signal delay for bus trip $i$;

$B T S_{-} G E_{i}, P T S_{-} G E_{i}$ : expected bus (and passenger) time savings due to a green extension phase for bus trip $i$;

$B T S_{-} E G_{i}, P T S_{-} E G_{i}$ : expected bus (and passenger) time savings due to an early green phase for bus trip $i$.

The bus arrival time probability at the intersection is calculated based on the upstream distance and bus travel speed probability distribution, which is estimated based on the observed bus stop-to-stop travel speeds. Because some of the bus stop-to-stop travel speed observations may include signal delay, these records will be excluded from the travel speed observations to estimate the non-stop bus travel speed distribution in the upstream distance for far-side segments or in the downstream distance for near-side segments. Assume the total number of bus travel speed observations for a bus stop-tostop segment at a certain time of day is $N$, and assume the ratio between the median red phase duration and the cycle length of the intersection is $\frac{R}{C}\left(0<\frac{R}{C}<1\right)$. First, the $N$ bus travel speed observations are ordered from the lowest to the highest. Then, the first $N \cdot \frac{R}{C}$ bus travel observations are removed. The remaining $N \cdot\left(1-\frac{R}{C}\right)$ speed observations are used to estimate the non-stop bus travel speed probability distribution. The non-stop bus 
speed probability distribution is estimated based on the frequencies of bus speed observations in each speed bin (1 mph increment). Therefore, the frequency of each speed bin ( $1 \mathrm{mph}$ increment) is the probability density of the speed. Because the non-stop bus travel speed distribution may vary by time of day, four non-stop bus travel speed probability distributions were estimated in four different times of day: AM peak hours (7-9 am), Mid-day (9 am-4 pm), PM peak hours (4-6 pm) and Evening (6 pm-7 am). The estimated non-stop bus travel speed histograms without signal delay for all stop-tostop segments in four times of day are shown in Appendix A. Because we do not have two separate bus travel speed distributions for the upstream distance and the downstream distance, we assume that the estimated bus travel speed distribution for the stop-to-stop segment applies to both the upstream and the downstream parts.

For a bus stop-to-stop segment at a certain time of day, define $f(v)$ as the probability density function for bus stop-to-stop travel speeds without signal delay, and define $v_{\text {mean }}$, $v_{\min }$ and $v_{\max }$ as the mean, minimum and maximum of these speeds. Then, the probability of a bus travels between speeds $v_{1}$ and $v_{2}$ is:

$$
P\left[v_{1} \leq v<v_{2}\right]=\int_{v_{1}}^{v_{2}} f(v) \mathrm{d} v
$$

Given the above input data and the probability function $f(v)$, an algorithm is developed to calculate the signal phase attributes output. The concept of the algorithm is explained below and the complete algorithm is shown in Appendix B. 
First, the Red attribute is a binary variable that indicates whether a bus trip encountered a red signal. The value of this variable is determined by comparing bus stop-to-stop travel time intervals with red phase intervals of an intersection.
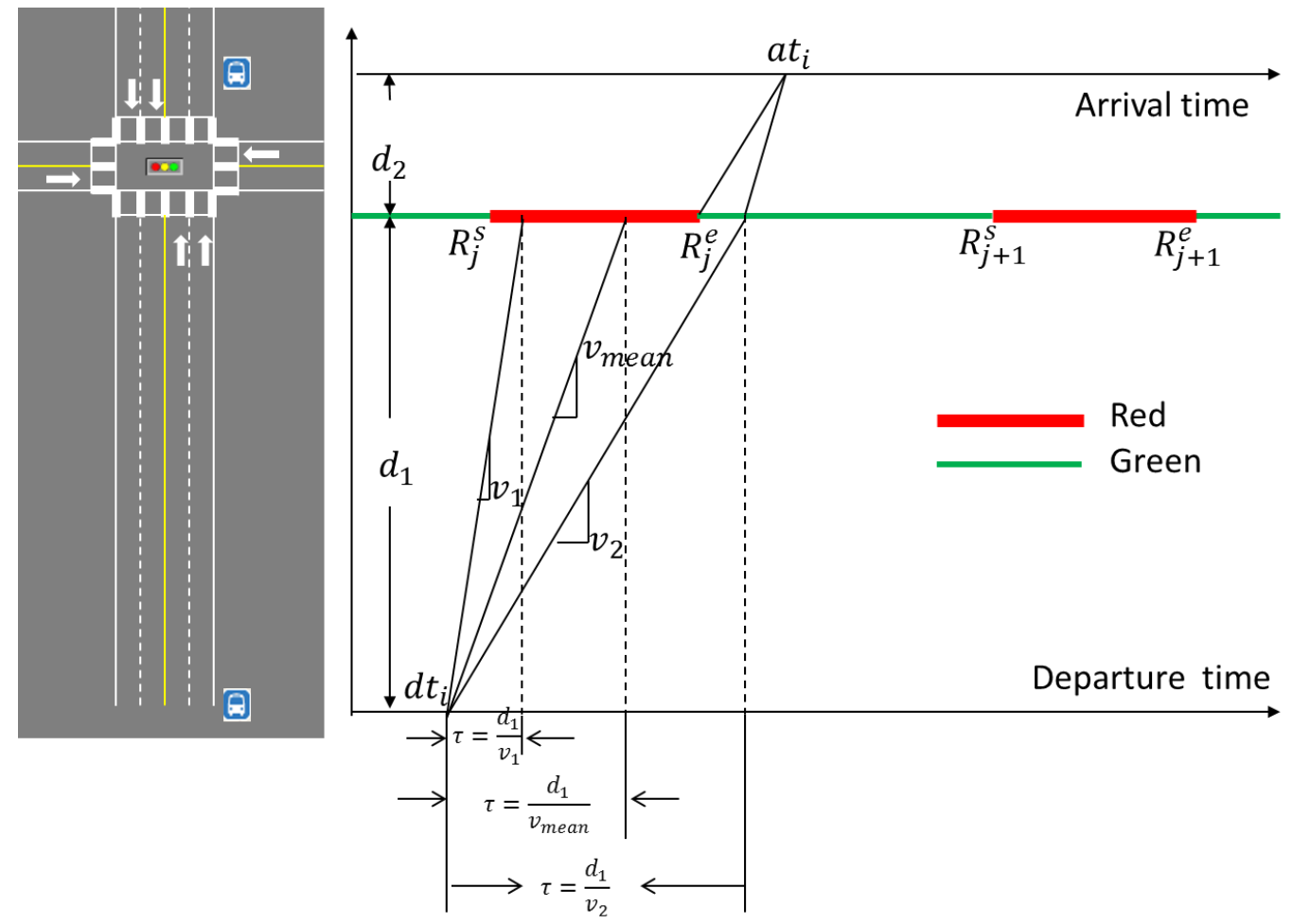

Figure 2-8 Time-space diagram of a bus that encounters a red signal delay

As shown in Figure 2-8, define $\tau$ as the travel time from the upstream bus stop to the intersection, $\tau(v)=\frac{d_{1}}{v}$. The Red variable is equal to 1 for bus trip $i$ if the following conditions are met by any red phase interval $j$ : 


$$
\begin{gathered}
d t_{i}+\tau \leq R_{j}^{e}<a t_{i} \\
R_{j}^{S}<d t_{i}+\tau
\end{gathered}
$$

The above equations mean that a bus $i$ encountered a red signal delay if its arrival time at the intersection $\left(d t_{i}+\tau\right)$ is before the end time $\left(R_{j}^{e}\right)$ of a red phase $j$, and its arrival time at the downstream stop $\left(a t_{i}\right)$ is after the end time $\left(R_{j}^{e}\right)$ of the red phase $j$. Another logical constraint is that the arrival time of bus $i$ at the intersection $\left(d t_{i}+\tau\right)$ should be after the start time $\left(R_{j}^{S}\right)$ of the red phase $j$; otherwise, the bus $i$ would not encounter a red signal. It is possible to state that the logical conditions must be met to determine whether a bus encountered a red signal or not, but the travel time $\tau(v)$ is unknown. According to Figure $2-8$, if we use $\tau=\frac{d_{1}}{v_{1}}, \operatorname{Red}_{i}=1$ is correct if the actual travel speed is $v_{1}$; it is wrong if the actual travel speed is $v_{1}$. Similarly, if we use $\tau=\frac{d_{1}}{v_{2}}, \operatorname{Red}_{i}=0$ is correct if the actual travel speed is $v_{2}$, but incorrect if the actual travel speed is $v_{1}$. Therefore, mean travel speed $\left(v_{\text {mean }}\right)$ at each segment is used to determine the travel time $\tau=\frac{d_{1}}{v_{\text {mean }}}$. 

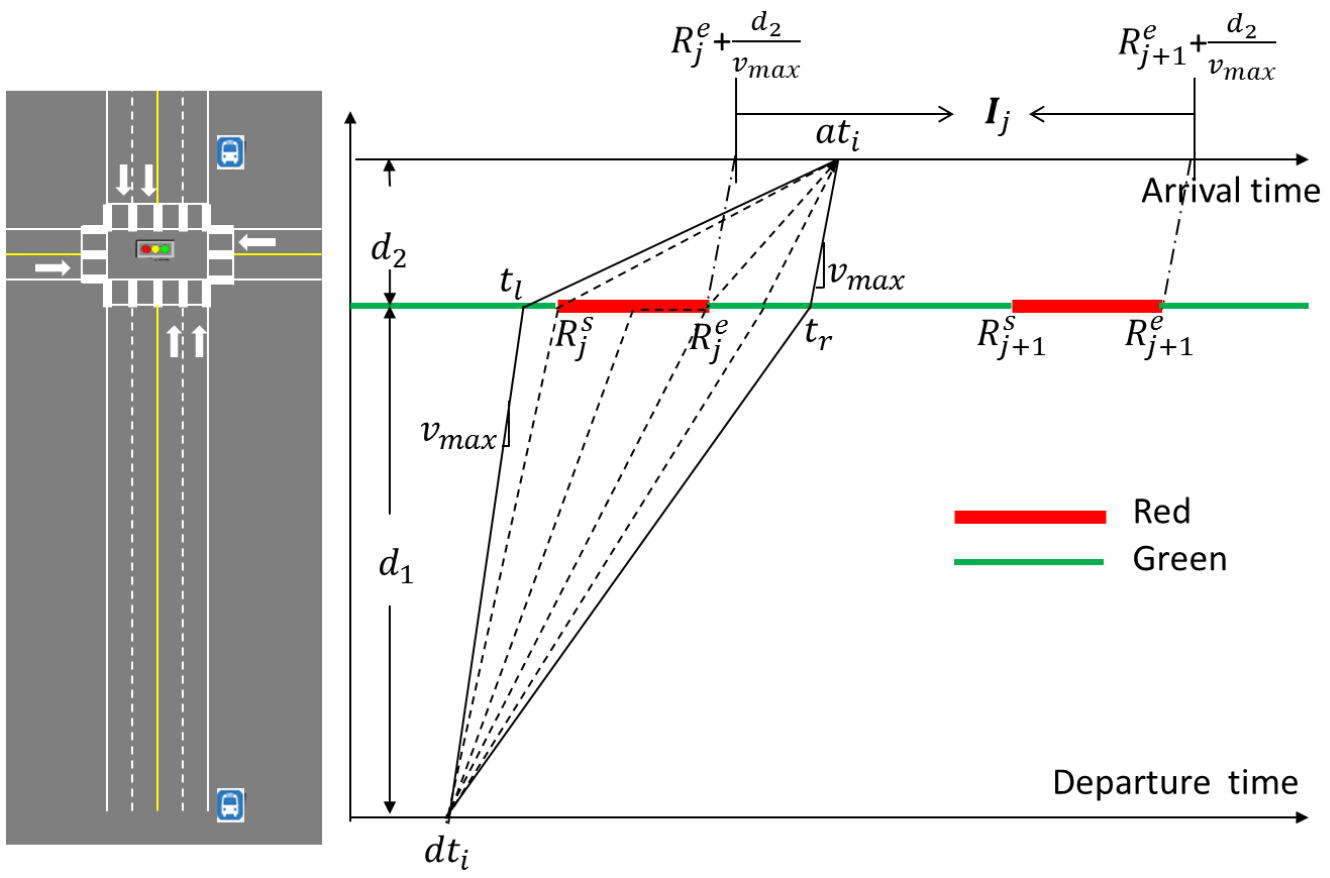

(a)
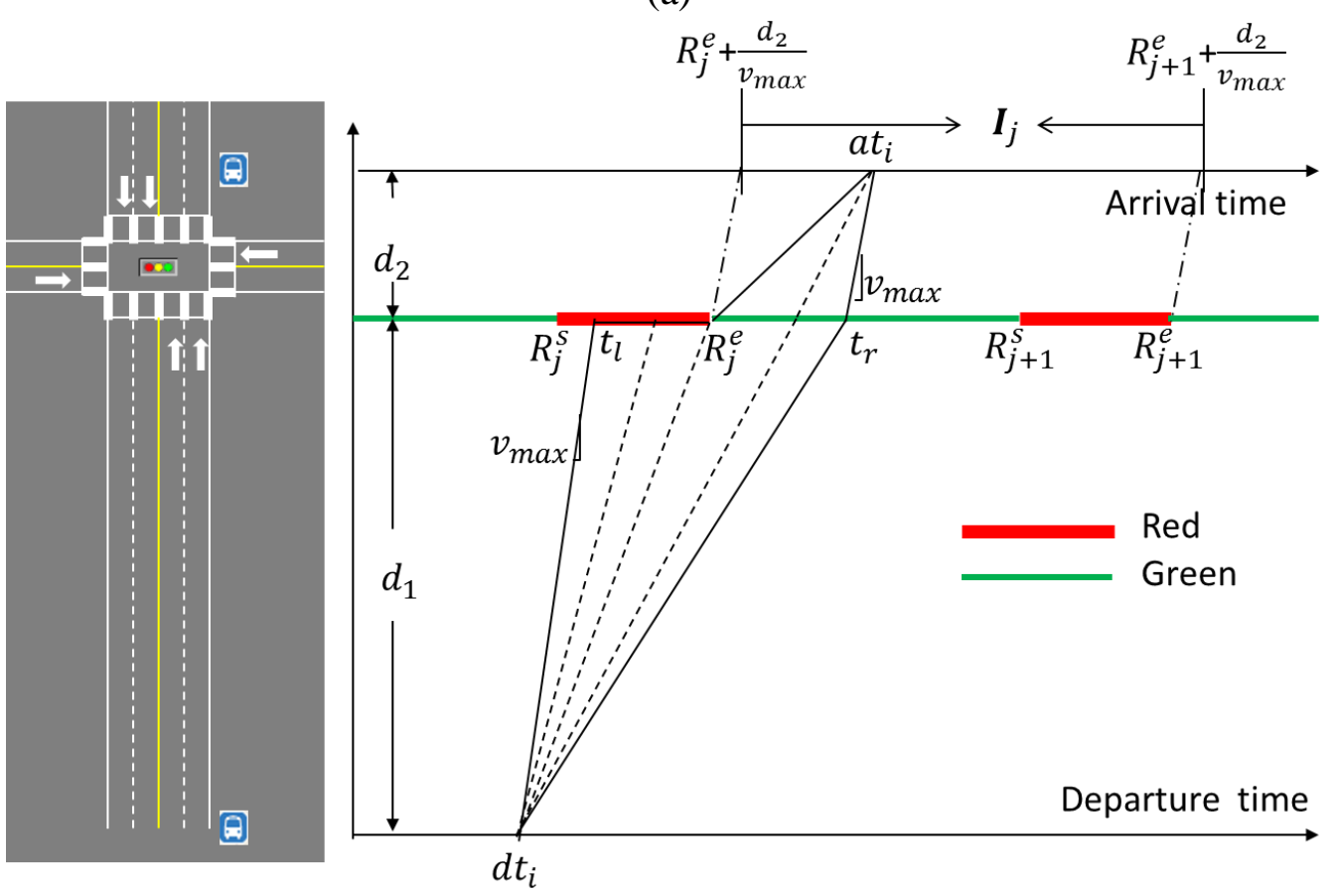

(b) 

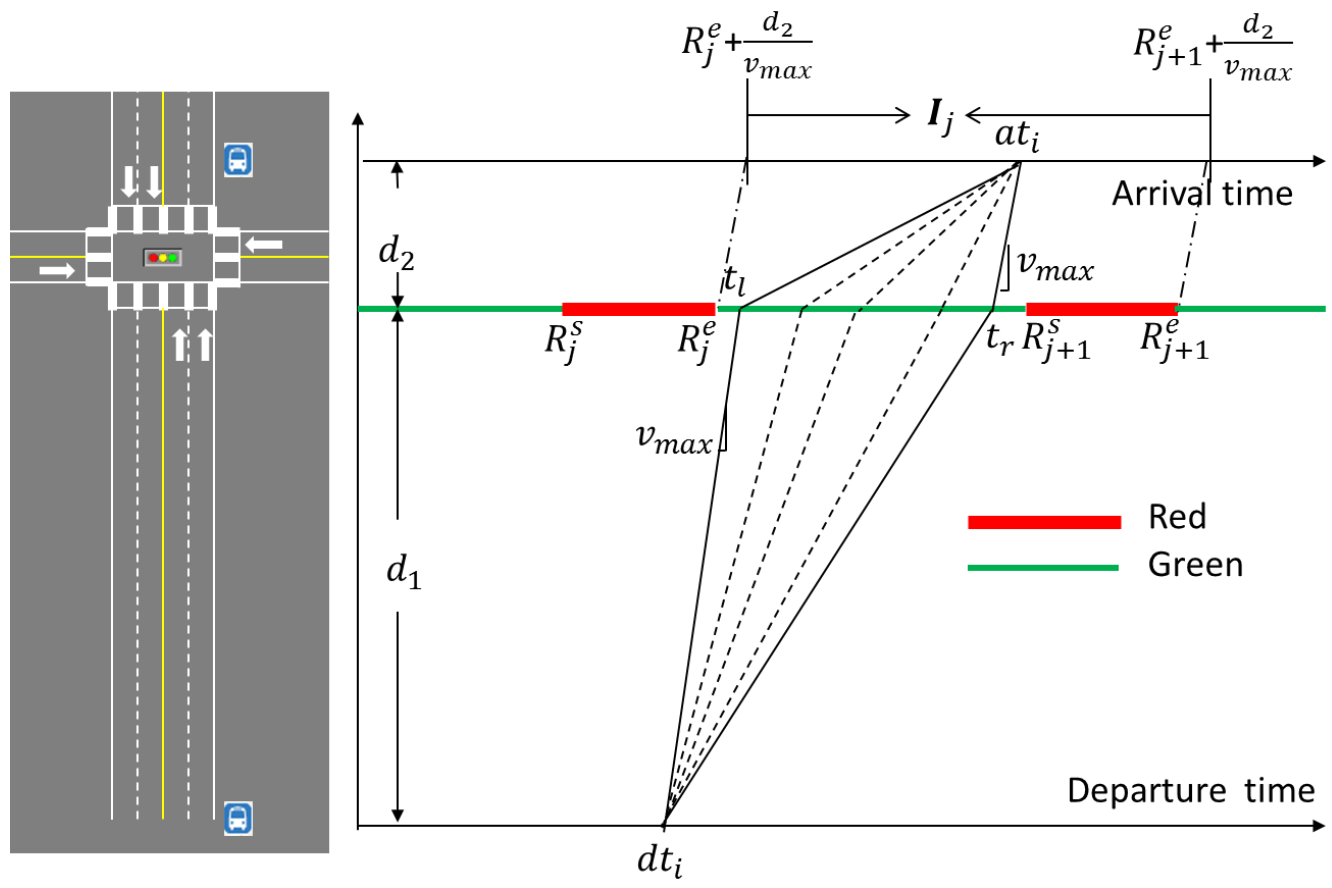

(c)
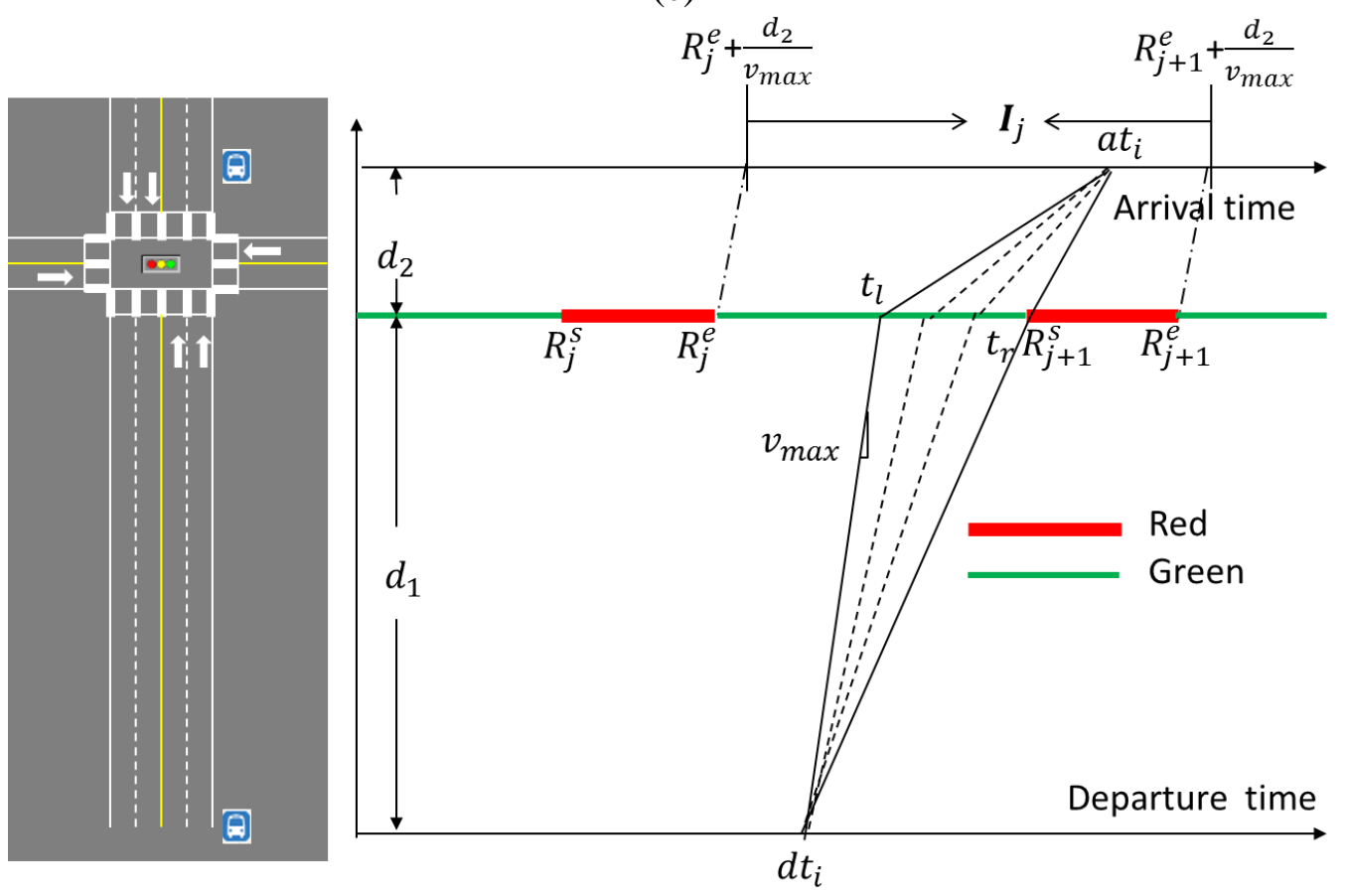

(d)

Figure 2-9 Feasible bus stop-to-stop trip trajectories 
Figure 2-9 (a) - (d) show some bus stop-to-stop trajectories in time-space diagrams. For a given cycle $j$, denote $\boldsymbol{I}_{j}$ as the set of bus trips that arrived at the arrival stop during the time interval $\left(R_{j}^{e}+\frac{d_{2}}{v_{\max }}, R_{j+1}^{e}+\frac{d_{2}}{v_{\max }}\right)$, then:

$$
\begin{gathered}
\boldsymbol{I}_{j}=\left\{i \mid i \in \boldsymbol{I}, R_{j}^{e}+\frac{d_{2}}{v_{\max }}<a t_{i} \leq R_{j+1}^{e}+\frac{d_{2}}{v_{\max }}\right\} \\
\boldsymbol{I}_{j} \cap \boldsymbol{I}_{k} \equiv \emptyset, \text { for } \forall j \neq k ; j, k \in \boldsymbol{J}
\end{gathered}
$$

If there is a bus trip $i$ in cycle $j\left(\boldsymbol{I}_{j} \neq \emptyset\right)$, based on the red phase start time $\left(R_{j}^{S}\right)$ and end time $\left(R_{j}^{e}\right)$, as well as the departure time $\left(d t_{i}\right)$ and arrival time $\left(a t_{i}\right)$ of the bus trip $i$, four different bus trajectory boundaries may exist as shown in Figure 2-9 (a) - (d). A bus trajectory boundary is defined by $d t_{i}, t_{l}, a t_{i}$ and $t_{r} . t_{l}$ and $t_{r}$ are the earliest and latest possible times that bus trip $i$ could be at the intersection given $d t_{i}$ and $a t_{i}$. They are defined by the following equations:

$$
\begin{gathered}
t_{l}=\max \left\{a t_{i}-\frac{d_{2}}{v_{\text {min }}}, d t_{i}+\frac{d_{1}}{v_{\text {max }}}\right\} \\
t_{r}=\min \left\{a t_{i}-\frac{d_{2}}{v_{\text {max }}}, d t_{i}+\frac{d_{1}}{v_{\text {min }}}, R_{j+1}^{s}\right\}
\end{gathered}
$$

Figure 2-9 (a) - (d) only show some examples when the feasible bus trajectory boundaries are determined by maximum speeds, the minimum speeds are usually not a constraint. There are only a few scenarios when the feasible boundary is determined by 
both maximum and minimum speeds. Also, Figure 2-9 (a) - (d) only show some examples when the feasible boundary is within one cycle, it is also possible that a feasible boundary spans two or more cycles (if a cycle failure occurs). Therefore, we define $\boldsymbol{J}_{i}^{\prime}$ as the set of cycles that maybe within the feasible boundary of bus $i$.

$$
\boldsymbol{J}_{i}^{\prime} \leftarrow\left\{k \mid k \leq j, \quad d t_{i} \leq G_{j}^{e}-\frac{d_{1}}{v_{\max }}\right\}
$$

Once $\boldsymbol{I}_{j}$ and $\boldsymbol{J}_{i}^{\prime}$ are defined, the bus signal phase attributes can be calculated. For each bus $i \in \boldsymbol{I}_{j}$ and each cycle $j \in \boldsymbol{J}_{i}^{\prime}$, the ways of calculating signal phase attributes are explained as below.

Prob_ $R_{i}$ can be estimated by the sum of speed probabilities that could lead bus $i$ arrive at the intersection during the red phases, conditional on the sum of all feasible speed probabilities. Therefore,

$$
{\operatorname{Prob} \_R_{i}}_{=} \sum_{j \in J_{i}^{\prime}} \frac{P\left[\frac{d_{1}}{R_{j}^{e}-d t_{i}} \leq v<\frac{d_{1}}{\max \left\{R_{j}^{S}, t_{l}\right\}-d t_{i}}\right]}{P\left[\frac{d_{1}}{t_{r}-d t_{i}} \leq v<\frac{d_{1}}{t_{l}-d t_{i}}\right]}
$$

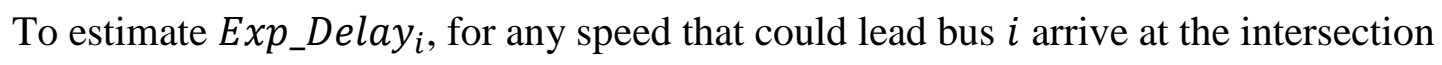
during the red phase, $v \in\left(\frac{d_{1}}{\max \left\{R_{j}^{S}, t_{l}\right\}-d t_{i}}, \frac{d_{1}}{R_{j}^{e}-d t_{i}}\right)$, the red signal delay is: $R_{j}^{e}-\left(d t_{i}+\frac{d_{1}}{v}\right)$. 
Given the speed probability density function, $f(v)$, the total expected delay can be estimated by:

$$
\text { Exp_Delay }_{i}=\sum_{j \in J_{i}^{\prime}} \frac{\int_{\frac{d_{1}}{R_{j}^{e}-d t_{i}}}^{\frac{d_{1}}{\max \left\{R_{j}^{s}, t_{l}\right\}-d t_{i}}} f(v) \cdot\left(R_{j}^{e}-d t_{i}-\frac{d_{1}}{v}\right) \mathrm{d} v}{P\left[\frac{d_{1}}{t_{r}-d t_{i}} \leq v<\frac{d_{1}}{t_{l}-d t_{i}}\right]}
$$

$P r o b_{-} G_{i}$ can be estimated similarly as $P r o b_{-} R_{i}$ :

$$
P r o b \_G_{i}=\sum_{j \in J_{i}^{\prime}} \frac{P\left[\frac{d_{1}}{\min \left\{G_{j}^{e}, t_{r}\right\}-d t_{i}} \leq v<\frac{d_{1}}{G_{j}^{S}-d t_{i}}\right]}{P\left[\frac{d_{1}}{t_{r}-d t_{i}} \leq v<\frac{d_{1}}{t_{l}-d t_{i}}\right]}
$$

Therefore, $P r o b_{-} R_{i}+P_{r o b} G_{i} \equiv 1$, if there is no TSP phase.

If there is a TSP phase in a cycle $j$, the probability of bus $i$ arriving at the intersection during the TSP phase and the expected time savings of the bus and the onboard passengers can also be estimated.

1. If there is an EG phase in cycle $j$;

Figure 2-10 shows an example of the probabilistic bus trajectory during a cycle with an EG phase. Depending on the relationships between $t_{l}, E G_{j}^{S}, t_{r}$ and $E G_{j}^{e}$, the range of 
speeds that could lead bus $i$ arrive at the intersection during the early green phase is $\left(\max \left\{E G_{j}^{s}, t_{l}\right\}, \min \left\{E G_{j}^{e}, t_{r}\right\}\right) ;$ therefore, $P_{0} b_{-} E G_{i}$ can be estimated by:

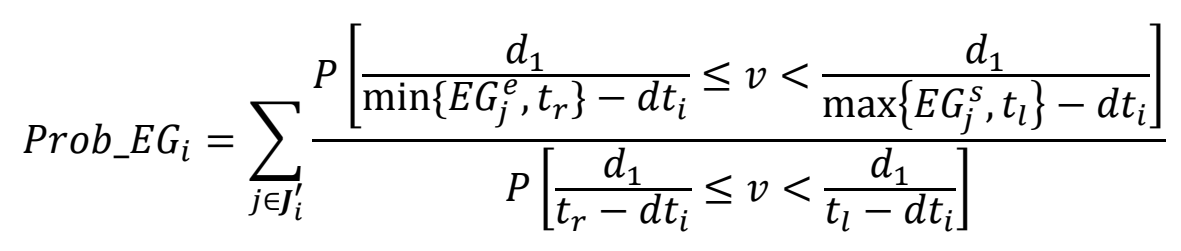
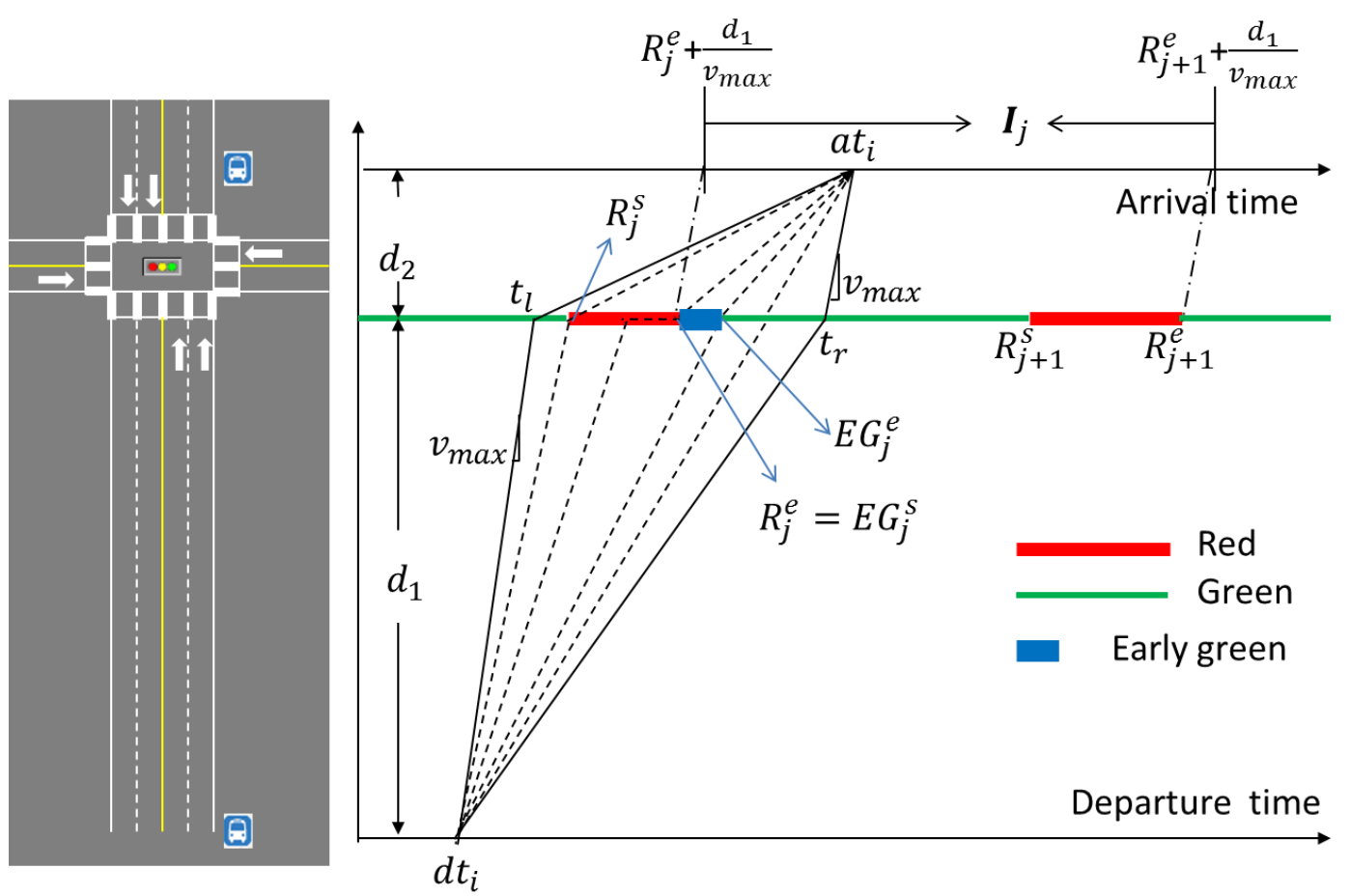

Figure 2-10 Feasible bus stop-to-stop trip trajectories with an early green phase

Figure 2-10 shows one scenario where $t_{l}<E G_{j}^{S}$ and $E G_{j}^{e}<t_{r}$, but the above equation works for the other three scenarios: 1) $t_{l}<E G_{j}^{s}$ and $E G_{j}^{e} \geq t_{r}$;2) $t_{l} \geq E G_{j}^{s}$ and $E G_{j}^{e} \geq$ $t_{r}$; 3) $t_{l} \geq E G_{j}^{s}$ and $E G_{j}^{e}<t_{r}$. 
If $E G_{j}^{S}<t_{l}$, bus $i$ is impossible to arrive at the intersection during the preceding red phase of this early green phase, the time saving for bus $i$ traveling at speed $v \in$ $\left(\frac{d_{1}}{t_{l}-d t_{i}}, \frac{d_{1}}{\min \left\{E G_{j}^{e}, t_{r}\right\}-d t_{i}}\right)$ is $\min \left\{E G_{j}^{e}, t_{r}\right\}-\left(d t_{i}+\frac{d_{1}}{v}\right)$. If $E G_{j}^{s} \geq t_{l}$, bus $i$ is also likely to arrive at the intersection during the preceding red phase of this early green phase.

Therefore, time saving for bus $i$ traveling at speed $v \in\left(\frac{d_{1}}{E G_{j}^{s}-d t_{i}}, \frac{d_{1}}{t_{l}-d t_{i}}\right)$ is $E G_{j}^{e}-E G_{j}^{s}$.

Therefore, $B T S_{-} E G_{i}$ and $P T S_{-} E G_{i}$ can be estimated by:

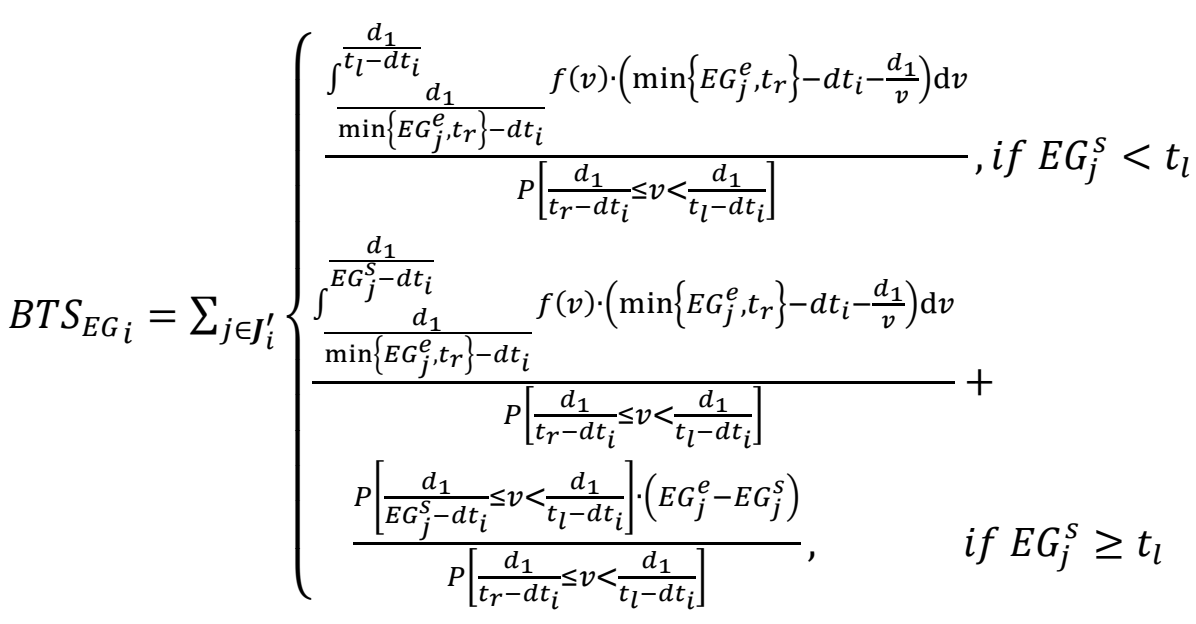

$$
\begin{aligned}
& P T S_{-} E G_{i}=\sum_{j \in J_{i}^{\prime}} B T S_{-} E G_{i} \cdot \operatorname{load}_{i}
\end{aligned}
$$

2. If there is a GE phase in cycle $j$.

Figure 2-11 shows an example of the probabilistic bus trajectory during a cycle with a GE phase. Because bus $i$ could benefit from a green extension phase only when it arrives at the intersection during the green extension phase, depending on the relationships 
between $t_{l}, G E_{j}^{s}, t_{r}$ and $G E_{j}^{e}$, bus $i$ will benefit from GE phase if it travels with speed $v \in\left(\frac{d_{1}}{\min \left\{G E_{j}^{e}, t_{r}\right\}-d t_{i}}, \frac{d_{1}}{\max \left\{G E_{j}^{s}, t_{l}\right\}-d t_{i}}\right)$, therefore:

$$
P r o b \_G E_{i}=\sum_{j \in J_{i}^{\prime}} \begin{cases}P\left[\frac{d_{1}}{\min \left\{G E_{j}^{e}, t_{r}\right\}-d t_{i}} \leq v<\frac{d_{1}}{\max \left\{G E_{j}^{S}, t_{l}\right\}-d t_{i}}\right] \\ P\left[\frac{d_{1}}{t_{r}-d t_{i}} \leq v<\frac{d_{1}}{t_{l}-d t_{i}}\right] & \text { if } t_{r} \geq G E_{j}^{S} \\ 0 & \text { if } t_{r}<G E_{j}^{S}\end{cases}
$$
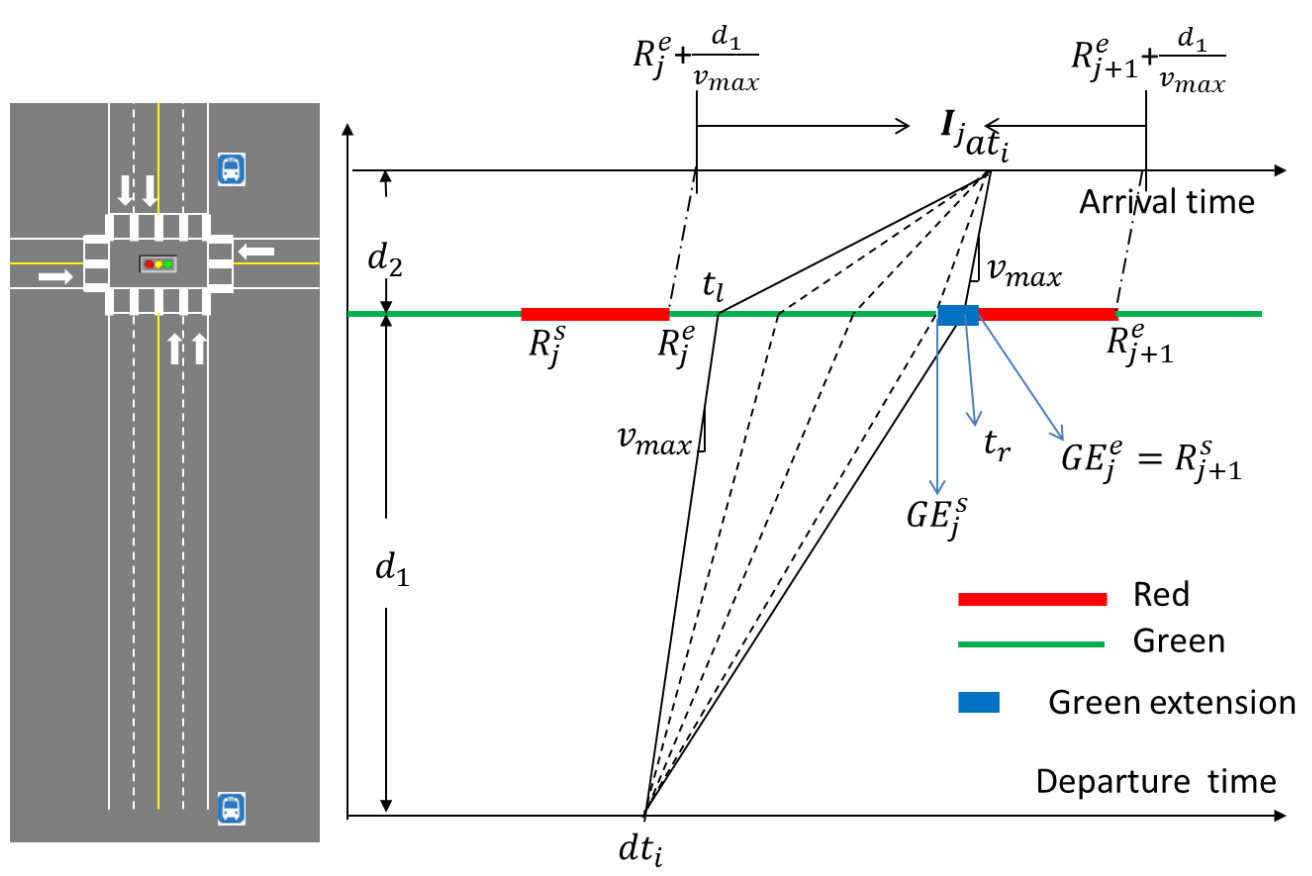

Figure 2-11 Feasible bus stop-to-stop trip trajectories with a green extension phase

Similarly, Figure 2-11 shows one scenario where $t_{l}<G E_{j}^{S}$ and $G E_{j}^{e}>t_{r}$, but the above equation works for the other three scenarios: 1) $t_{l}<G E_{j}^{S}$ and $G E_{j}^{e} \leq t_{r}$;2) $t_{l} \geq G E_{j}^{S}$ and $\left.G E_{j}^{e} \leq t_{r} ; 3\right) t_{l} \geq G E_{j}^{s}$ and $G E_{j}^{e}>t_{r}$ 
If bus $i$ travels with speed $v \in\left(\frac{d_{1}}{\min \left\{G E_{j}^{e}, t_{r}\right\}-d t_{i}}, \frac{d_{1}}{\max \left\{G E_{j}^{s}, t_{l}\right\}-d t_{i}}\right)$, the time saving is $R_{j+1}^{e}-\left(d t_{i}+\frac{d_{1}}{v}\right)$; therefore, $B T S_{-} G E_{i}$ and PTS $G E_{i}$ can be estimated by:

$$
\begin{aligned}
& B T S_{-} G E_{i}=\sum_{j \in J_{i}^{\prime}} \begin{cases}\frac{\int_{\frac{d_{1}}{\max \left\{G E_{j}^{S}, t_{l}\right\}-d t_{i}}}^{\frac{d_{1}}{\min \left\{G E_{j}^{e}, t_{r}\right\}-d t_{i}}} f(v) \cdot\left(R_{j+1}^{e}-d t_{i}-\frac{d_{1}}{v}\right) \mathrm{d} v}{P\left[\frac{d_{1}}{t_{r}-d t_{i}} \leq v<\frac{d_{1}}{t_{l}-d t_{i}}\right]} & \text {, if } t_{r} \geq G E_{j}^{S} \\
0 & \text {,if } t_{r}<G E_{j}^{S}\end{cases} \\
& P T S_{-} G E_{i}=\sum_{j \in J_{i}^{\prime}} B T S_{-} G E_{i} \cdot \operatorname{load}_{i}
\end{aligned}
$$

\subsection{Summary}

This chapter first introduced the study corridor, bus stops and signal timing characteristics, and then a detailed description of the three databases is provided, followed by an overview of the research methodology. Finally, this chapter explained how the three databases were integrated into one bus stop-to-stop trip database and how each of the attributes was calculated. The algorithm developed in this study is one of the important contributions of this research. This new database will be used for bus travel time regression analysis and TSP performance evaluation. 


\section{BUS TRAVEL TIME MODELING}

\subsection{Chapter Outline}

This chapter presents empirical analyses measuring the effects of traffic signal delay, traffic conditions and bus stop location on bus stop-to-stop travel time. It begins with descriptions of multiple linear regression models and descriptive statistics of the variables used in the analysis. Then, variable descriptions and model specifications are presented, followed by a discussion of the results.

\subsection{Variable Descriptions and Model Specifications}

\subsubsection{Variable descriptions}

Twelve far-side segments and six near-side segments were used to model bus stop-to-stop travel time at the intersections between $26^{\text {th }}$ Ave. and $72^{\text {nd }}$ Ave. on Powell Blvd. Table 3-1 shows the descriptions of the variables that will be used in the regression models. 
Table 3-1 Description of variables

Description

\section{Dependent variables}

Departure-to-arrival time

Arrival-to-arrival time
Arrival time at downstream stop minus departure time at upstream stop (seconds)

Arrival time at downstream stop minus arrival time at upstream stop (seconds)

\section{Independent variables}

Travel impedance variables

Distance

Traffic volume

Peak

Signal delay variables

Red

Red time

$\mathrm{RC}$ ratio
Travel distance between upstream and downstream stops (miles)

Thousands of through vehicles per hour (1000 veh/hour)

$=1$, if in 7-9 am in WB direction or 4-6 pm in EB direction

$=1$, if the bus encountered a red signal

Red phase duration (seconds) if the bus encountered a red signal

The ratio between the median red phase duration for the bus movement direction and the median cycle length at an intersection

Departure stop activity variables

Ons

Number of boarding passengers at the departure stop

Offs

Number of alighting passengers at the departure stop

Lift

Number of lift uses at the departure stop

Departure delay

Actual departure time - scheduled departure time (minutes)

Skip

$=1$, if a bus skipped the departure stop;

Segment characteristics variables

Near

$=1$, if the stop-to-stop segment is a near-side segment

As introduced in Chapter 2, Departure time is the time when a bus leaves the 50-foot stop

circle. However, since near-side stops are usually less than 50 feet upstream of an

intersection stop bar, signal delay may not be included in the departure-to-arrival time for

near-side segments. Therefore, the departure-to-arrival time was used as the dependent

variable to study bus stop-to-stop travel time only for far-side segments; the arrival-to- 
arrival time was used as the dependent variable to study bus stop-to-stop travel time for both far-side segments and near-side segments.

In this chapter, both the departure-to-arrival time model and the arrival-to-arrival time model will be estimated for far-side segments, and arrival-to-arrival time model will be estimated for all segments together. These models are called pooled regression models. Furthermore, the departure-to-arrival time model and arrival-to-arrival time model will be estimated for each individual stop-to-stop segment to investigate the impacts of intersection and segment geometric characteristics. These models are called individual regression models.

In Table 3-1, the variable distance is defined as the travel distance between the upstream stop and the downstream stop measured in miles. This variable is introduced to control for the effect of distance on bus stop-to-stop travel time. This variable will be used in the pooled regression models but not the individual regression models, because distance is a constant in each stop-to-stop segment.

The traffic volume variable is included to measure the effect of traffic conditions on bus stop-to-stop travel time. Traffic volume for a bus trip is the equivalent number of through vehicles per hour in the bus travel direction in the 15-minute interval, to which the bus arrival time at the downstream stop belongs. It is expected that in uncongested traffic conditions, as traffic volumes increase, bus stop-to-stop travel time may increase due to more lane changing activities and/or increasing queues. However, in transition periods, a 
traffic volume decrease may indicate congestion formation, and therefore bus travel time may increase. Conversely, a traffic volume increase may indicate congestion dissipation, and bus travel time may decrease. Therefore, both positive and negative relationships may exist between traffic volume and bus stop-to-stop travel time.

The peak dummy variable is also introduced to control for the effect of traffic conditions on bus travel time. Powell Blvd. is a typical commute corridor with high traffic demand in the AM peak hours in the inbound (WB) direction and in the PM peak hours in the outbound direction (EB). This variable is 1 if a bus travels in the WB direction between 7 am and 9 am or in the EB direction between $4 \mathrm{pm}$ and $6 \mathrm{pm}$. The effect of the peak variable is expected to be positive (increase bus delay). It is also expected that the traffic volume impact on bus stop-to-stop travel time differs between peak and off-peak hours. This effect can be tested by including the interaction term between the peak variable and the traffic volume variable. Depending on how frequently and severely traffic congestion occurs at different intersections at different times of day, the effect of traffic conditions on bus stop-to-stop travel time may be positive, neutral or negative.

The red dummy variable represents whether a bus encountered a red signal delay when travelling through an intersection. The method of calculating this dummy variable is explained in Chapter 2. This variable is included to study the average effect of signal delay on bus stop-to-stop travel time. Bus signal delay consists of three components as shown in Figure 3-1: 1) waiting time for a red signal; 2) deceleration/acceleration delay; 3) queuing effect delay. The expected waiting time for a red signal will be equal to half of 
the red phase duration if the bus arrival time at an intersection is randomly distributed. The deceleration and acceleration delay is usually constant and independent of the red phase duration. Queuing effect delay increases with increasing queue length in front of a bus. The red dummy variable will be used in the individual regression models to evaluate the average effect of signal delay including all three components. The expected coefficient of this variable will be more than half of the average red phase duration for each segment. The red dummy variable will not be used in the pooled regression models because the estimated coefficient would represent an average signal delay across all stopto-stop segments without relation to signal phase characteristics.

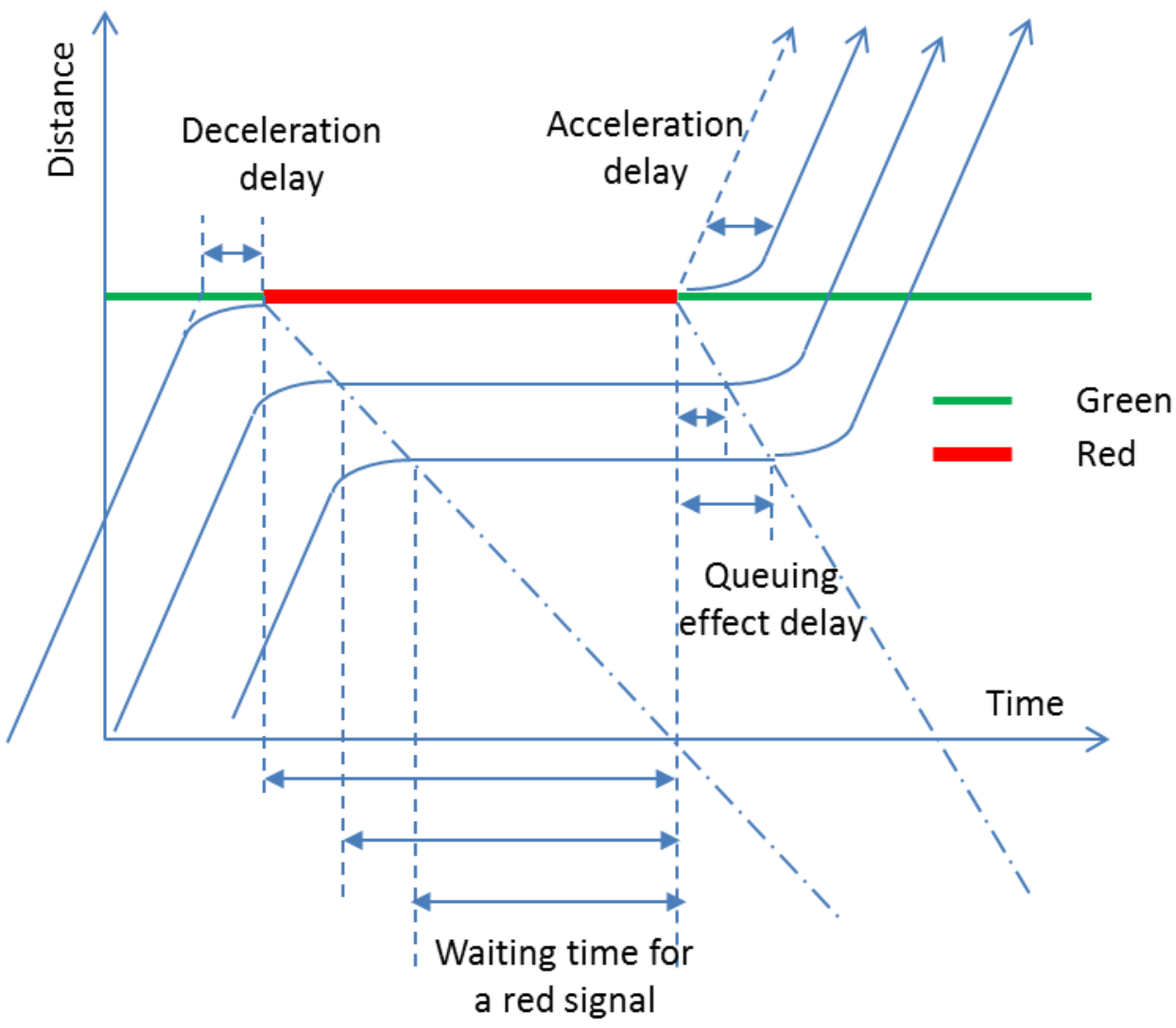

Figure 3-1 Bus red phase delay components 
The red time variable is the actual red phase duration in seconds if a bus encountered a red signal. Therefore, if a bus encountered a red signal in cycle $j$, red time is equal to $R_{j}^{e}-R_{j}^{S}$, where $R_{j}^{S}$ and $R_{j}^{e}$ are the start time and end time of the red phase in cycle $j$. If a bus did not experience a red signal, red time equals 0 . This variable is used in the pooled regression models to measure the signal delay in proportion to the red phase duration.

The red time variable represents the average ratio between experienced signal delay and red phase duration. However, this ratio is expected to be higher for segments with longer red phase durations due to additional queuing effects. Therefore, the $R C$ ratio variable is introduced to capture the additional queuing delay at segments with long red phase durations. Some intersections with high cross street traffic volumes have long red phase durations for the EB and WB directions, such as intersections at $26^{\text {th }}, 39^{\text {th }}, 50^{\text {th }}$ and $52^{\text {nd }}$ Ave. on Powell Blvd.

Ons, offs, and lift are continuous variables that measure the numbers of boarding passengers, alighting passengers and lift use. They are included to control for the impacts of passenger activities on departure stop dwell time, and therefore on bus arrival-toarrival time. They are expected to be positive.

The departure delay variable is included to capture whether schedule delay at the departure stop has an impact on bus driver behavior, and consequently has an impact on bus stop-to-stop travel time. Departure delay is the difference between the actual 
departure time and the scheduled departure time at the departure stop in a stop-to-stop segment. This variable is measured in minutes to scale up the estimated coefficient. It is expected that late buses travel faster than early buses. However, because bus travel distance is too short between two consecutive bus stops, the effect of departure delay may be very small or insignificant. This variable will only be used in the far-side segments departure-to-arrival time model, because departure delay is not accurately measured for near-side segments.

The skip dummy variable is included to distinguish whether a bus skipped the departure stop or not. It is expected to significantly affect the bus stop-to-stop travel time, because if a bus skips a bus stop, there will be no deceleration/acceleration delay or dwell time. Therefore, this variable is expected to be significant and negative in the bus arrival-toarrival time model. It is also expected to be significant and negative in the bus departureto-arrival time model but with a smaller absolute value of the coefficient, because if a bus skips the departure stop, only partial acceleration delay will be saved for the departure-toarrival time.

The near dummy variable is introduced to control for the effect of bus stop location on bus stop-to-stop travel time and to test whether other variables' effects on bus travel time differ between near-side segments and far-side segments.

Distance, $R C$ ratio and near variables are constants for each stop-to-stop segment, which explains bus travel time variance between stop-to-stop segments. All other variables are 
not constant in each stop-to-stop segment and they explain bus travel time variance within each stop-to-stop segment.

Other than the variables listed in Table 3-1, we originally tried to investigate the impact of bus stop bay at the departure stop and the arrival stop on bus stop-to-stop travel time. Because a bus bay at the departure stop may create additional delay for buses that try to merge to the traffic lane; a bus bay at the arrival stop may help a bus arrive at the stop a little earlier only when there is considerable traffic, this variable may be significantly negative or insignificant. However, there are only 18 bus stop-to-stop segments, and there are already three variables (distance, $R C$ ratio and near) in the model that explain between-segment variance, there is not enough degree of freedom to estimate the impacts of bus bay on stop-to-stop travel time.

\subsubsection{Model specifications}

Table 3-2 summarizes all the variables and their interactions that are used in the pooled regression models and individual regression models. The far-side segments departure-toarrival time model focuses on analyzing the pure effects of signal delay and traffic conditions on bus travel time, eliminating the effects of departure stop passenger activities. The far-side segments arrival-to-arrival time model is estimated to ensure that the estimated effects of signal delay and traffic conditions are not associated with the impacts of passenger activities at the departure stop. The all segments arrival-to-arrival time model tries to quantify the simultaneous effects of signal delay, traffic conditions, 
passenger activities, intersection and segment geometric characteristics, bus stop location type and their interactions on bus stop-to-stop travel time. Individual segment stop-tostop travel time model aims to reveal more details about how those effects vary across intersections and travel directions. Similarly, the departure-to-arrival time models at individual far-side segments are estimated to assess the different impacts of signal delay and traffic volume on bus travel time by eliminating the impacts of departure stop activities. The arrival-to-arrival time models at individual segments are estimated to validate the estimated coefficients and to compare the difference between far-side segments and near-side segments.

Table 3-2 Regression model specifications

\begin{tabular}{|c|c|c|c|c|c|}
\hline & \multicolumn{2}{|c|}{$\begin{array}{l}\text { Far-side } \\
\text { segments }\end{array}$} & \multirow{2}{*}{$\begin{array}{c}\text { All } \\
\text { segments }\end{array}$} & \multicolumn{2}{|c|}{$\begin{array}{c}\text { Individual } \\
\text { segments }\end{array}$} \\
\hline & $\begin{array}{l}\text { Departure } \\
\text {-to-arrival }\end{array}$ & $\begin{array}{c}\text { Arrival- } \\
\text { to-arrival }\end{array}$ & & $\begin{array}{l}\text { Departure } \\
\text {-to-arrival }\end{array}$ & $\begin{array}{c}\text { Arrival- } \\
\text { to-arrival }\end{array}$ \\
\hline Distance & $\bullet$ & $\bullet$ & - & & \\
\hline Distance*near & & & - & & \\
\hline Traffic volume & - & - & - & - & - \\
\hline Traffic volume*near & & & - & & \\
\hline Peak & - & - & - & - & - \\
\hline Peak*Traffic volume & & & & $\bullet$ & $\bullet$ \\
\hline Red & & & & $\bullet$ & $\bullet$ \\
\hline Red time & $\bullet$ & $\bullet$ & $\bullet$ & & \\
\hline Red time*near & & & $\bullet$ & & \\
\hline $\mathrm{RC}$ ratio & $\bullet$ & $\bullet$ & $\bullet$ & & \\
\hline $\mathrm{RC}$ ratio*near & & & $\bullet$ & & \\
\hline Ons & & $\bullet$ & $\bullet$ & & $\bullet$ \\
\hline Ons*near & & & $\bullet$ & & \\
\hline Offs & & - & - & & - \\
\hline Offs*near & & & $\bullet$ & & \\
\hline Lift & & $\bullet$ & $\bullet$ & & $\bullet$ \\
\hline Lift*near & & & $\bullet$ & & \\
\hline Departure delay & $\bullet$ & $\bullet$ & & $\bullet$ & \\
\hline Skip & • & $\bullet$ & $\bullet$ & $\bullet$ & $\bullet$ \\
\hline Near & & & $\bullet$ & & \\
\hline
\end{tabular}




\subsubsection{Variable descriptive statistics}

Table 3-3 presents a summary of segment characteristic variables. The number of observed bus stop-to-stop trips in each segment ranges between 2,760 and 2,930.

Segment distance ranges between 0.1 and 0.24 miles. The mean distances of far-side segments and all segments are 0.13 and 0.14 miles, respectively. $\mathrm{RC}$ ratios range between 0.1 and 0.55 . Nine out of twelve far-side segments have a bus bay at the arrival stop. Six out of eighteen segments are near-side segments. The total numbers of bus trips for farside segments and all stop-to-stop segments are 34,070 and 51,307, respectively.

Table 3-3 Stop-to-stop segment characteristics

\begin{tabular}{lcccc}
\hline & $\begin{array}{c}\text { Distance } \\
\text { (miles) }\end{array}$ & $\begin{array}{c}\text { Near-side } \\
\text { segment }\end{array}$ & $\begin{array}{c}\text { RC } \\
\text { ratio }\end{array}$ & $\begin{array}{c}\text { Number of } \\
\text { observations }\end{array}$ \\
\hline $26^{\text {th }} \mathrm{EB}$ & 0.18 & 1 & 0.31 & 2936 \\
$26^{\text {th }} \mathrm{WB}$ & 0.13 & 1 & 0.31 & 2757 \\
$33^{\text {rd }} \mathrm{EB}$ & 0.12 & 1 & 0.14 & 2927 \\
$33^{\text {rd }} \mathrm{WB}$ & 0.14 & 0 & 0.14 & 2756 \\
$39^{\text {th }} \mathrm{EB}$ & 0.16 & 0 & 0.55 & 2938 \\
$39^{\text {th }} \mathrm{WB}$ & 0.11 & 0 & 0.55 & 2760 \\
$42^{\text {nd }} \mathrm{EB}$ & 0.24 & 1 & 0.21 & 2937 \\
$43^{\text {rd }} \mathrm{WB}$ & 0.13 & 1 & 0.21 & 2757 \\
$50^{\text {th }} \mathrm{EB}$ & 0.16 & 0 & 0.42 & 2935 \\
$50^{\text {th }} \mathrm{WB}$ & 0.14 & 0 & 0.47 & 2762 \\
$52^{\text {nd }} \mathrm{EB}$ & 0.1 & 0 & 0.35 & 2928 \\
$52^{\text {nd }} \mathrm{WB}$ & 0.16 & 0 & 0.28 & 2761 \\
$65^{\text {th }} \mathrm{EB}$ & 0.14 & 0 & 0.13 & 2920 \\
$65^{\text {th }} \mathrm{WB}$ & 0.1 & 0 & 0.13 & 2760 \\
$69^{\text {th }} \mathrm{EB}$ & 0.11 & 0 & 0.10 & 2932 \\
$71^{\text {st }} \mathrm{EB}$ & 0.13 & 0 & 0.15 & 2930 \\
$72^{\text {nd }} \mathrm{EB}$ & 0.18 & 1 & 0.18 & 2933 \\
$72^{\text {nd }} \mathrm{WB}$ & 0.17 & 0 & 0.16 & 2761 \\
\hline Far-side segments & 0.13 & NA & 0.29 & 34070 \\
\hline All segments & 0.14 & 0.34 & 0.27 & 51307 \\
\hline
\end{tabular}


Table 3-4 Variable descriptive statistics

\begin{tabular}{|c|c|c|c|c|c|c|c|c|c|c|c|c|c|c|c|c|c|c|c|c|}
\hline & \multicolumn{2}{|c|}{$\begin{array}{c}\text { Departure- } \\
\text { to-arrival } \\
\text { time (sec) }\end{array}$} & \multicolumn{2}{|c|}{$\begin{array}{l}\text { Arrival- } \\
\text { to-arrival } \\
\text { time (sec) }\end{array}$} & \multicolumn{2}{|c|}{$\begin{array}{c}\text { Traffic } \\
\text { Volume } \\
\text { (1000vph) }\end{array}$} & \multicolumn{2}{|c|}{ Peak } & \multicolumn{2}{|c|}{ Red } & \multicolumn{2}{|c|}{ Ons } & \multicolumn{2}{|c|}{ Offs } & \multicolumn{2}{|c|}{ Lift } & \multicolumn{2}{|c|}{$\begin{array}{c}\text { Departure } \\
\text { delay } \\
\text { (min) }\end{array}$} & \multicolumn{2}{|c|}{ Skip } \\
\hline & mean & sd & mean & $\mathrm{sd}$ & mean & $\mathrm{sd}$ & mean & $\mathrm{sd}$ & mean & sd & mean & sd & mean & sd & mean & $\mathrm{sd}$ & mean & $\mathrm{sd}$ & mean & $\mathrm{sd}$ \\
\hline $26^{\text {th }} \mathrm{EB}$ & - & - & 66 & 30 & 1.0 & 0.5 & 0.2 & 0.4 & 0.5 & 0.5 & 1.9 & 3.6 & 4.1 & 18.9 & 0.0 & 0.1 & - & - & 0.2 & 0.4 \\
\hline $26^{\text {th }} \mathrm{WB}$ & - & - & 45 & 26 & 1.1 & 0.4 & 0.2 & 0.4 & 0.3 & 0.5 & 2.1 & 4.3 & 2.7 & 17.3 & 0.0 & 0.1 & - & - & 0.2 & 0.4 \\
\hline $33^{\mathrm{rd}} \mathrm{EB}$ & - & - & 35 & 17 & 1.2 & 0.5 & 0.2 & 0.4 & 0.2 & 0.4 & 0.4 & 0.9 & 2.2 & 16.1 & 0.0 & 0.1 & - & - & 0.4 & 0.5 \\
\hline $39^{\text {th }} \mathrm{EB}$ & 51 & 27 & 58 & 29 & 0.9 & 0.4 & 0.2 & 0.4 & 0.4 & 0.5 & 0.3 & 0.8 & 1.6 & 13.7 & & & 1.5 & 3.3 & 0.6 & 0.5 \\
\hline $39^{\text {th }} \mathrm{WB}$ & 44 & 27 & 52 & 30 & 0.9 & 0.3 & 0.2 & 0.4 & 0.4 & 0.5 & 0.3 & 0.7 & 1.7 & 12.7 & 0.0 & 0.0 & 0.7 & 2.4 & 0.6 & 0.5 \\
\hline $42^{\text {nd }} E B$ & - & - & 48 & 19 & 0.9 & 0.4 & 0.2 & 0.4 & 0.3 & 0.5 & 0.3 & 0.7 & 2.0 & 15.3 & 0.0 & 0.0 & - & - & 0.5 & 0.5 \\
\hline $43^{\mathrm{rd}} \mathrm{WB}$ & - & - & 33 & 19 & 0.9 & 0.3 & 0.2 & 0.4 & 0.3 & 0.4 & 0.6 & 1.0 & 1.2 & 11.5 & 0.0 & 0.0 & - & - & 0.5 & 0.5 \\
\hline $50^{\text {th }} \mathrm{EB}$ & 43 & 23 & 49 & 26 & 0.7 & 0.3 & 0.2 & 0.4 & 0.3 & 0.5 & 0.3 & 0.9 & 1.5 & 12.8 & 0.0 & 0.1 & 1.9 & 3.3 & 0.6 & 0.5 \\
\hline $50^{\text {th }} \mathrm{WB}$ & 50 & 29 & 77 & 40 & 0.7 & 0.3 & 0.2 & 0.4 & 0.5 & 0.5 & 1.8 & 2.6 & 2.8 & 16.9 & 0.0 & 0.1 & 0.5 & 2.3 & 0.1 & 0.3 \\
\hline $52^{\text {nd }} W B$ & 37 & 18 & 48 & 23 & 0.9 & 0.4 & 0.2 & 0.4 & 0.2 & 0.4 & 0.6 & 1.0 & 2.1 & 13.7 & 0.0 & 0.0 & 0.1 & 2.3 & 0.4 & 0.5 \\
\hline $65^{\text {th }} \mathrm{EB}$ & 22 & 8 & 27 & 12 & 0.8 & 0.4 & 0.2 & 0.4 & 0.1 & 0.3 & 0.1 & 0.4 & 1.1 & 12.1 & 0.0 & 0.0 & 1.7 & 3.4 & 0.7 & 0.5 \\
\hline $65^{\text {th }} \mathrm{WB}$ & 19 & 7 & 30 & 16 & 0.8 & 0.3 & 0.2 & 0.4 & 0.1 & 0.3 & 0.6 & 1.0 & 1.2 & 11.1 & 0.0 & 0.0 & 0.6 & 2.2 & 0.5 & 0.5 \\
\hline $69^{\text {th }} \mathrm{EB}$ & 19 & 6 & 26 & 13 & 0.8 & 0.4 & 0.2 & 0.4 & 0.0 & 0.2 & 0.2 & 0.6 & 1.5 & 13.3 & 0.0 & 0.0 & 1.5 & 3.4 & 0.6 & 0.5 \\
\hline $71^{\text {st }} \mathrm{EB}$ & 23 & 10 & 30 & 16 & 0.9 & 0.4 & 0.2 & 0.4 & 0.1 & 0.3 & 0.2 & 0.5 & 1.0 & 11.0 & 0.0 & 0.1 & 1.4 & 3.4 & 0.6 & 0.5 \\
\hline $72^{\text {nd }} E B$ & - & - & 40 & 18 & 0.8 & 0.4 & 0.2 & 0.4 & 0.2 & 0.4 & 0.4 & 0.9 & 2.3 & 16.0 & 0.0 & 0.2 & - & - & 0.4 & 0.5 \\
\hline $72^{\text {nd }} W B$ & 30 & 10 & 37 & 15 & 0.8 & 0.3 & 0.2 & 0.4 & 0.1 & 0.3 & 0.4 & 0.8 & 0.7 & 8.7 & 0.0 & 0.1 & 0.7 & 2.1 & 0.6 & 0.5 \\
\hline $\begin{array}{l}\text { Far-side } \\
\text { segments }\end{array}$ & 33 & 22 & - & - & 0.8 & 0.4 & 0.2 & 0.4 & 0.2 & 0.4 & 0.5 & 0.2 & 1.8 & 13.5 & 0.0 & 0.1 & 1.2 & 2.9 & 0.5 & 0.5 \\
\hline $\begin{array}{l}\text { All } \\
\text { segments }\end{array}$ & - & - & 44 & 27 & 0.8 & 0.5 & 0.2 & 0.4 & 0.3 & 0.4 & 0.7 & 1.8 & 2.0 & 14.4 & 0.0 & 0.1 & - & - & 0.5 & 0.5 \\
\hline
\end{tabular}


Table 3-4 presents a summary of means and standard deviations of key variables for each individual segment and the average of far-side segments and all stop-to-stop segments. Departure-to-arrival time and departure delay for near-side segments are not available. Results show that the means and standard deviations of departure-to-arrival time and arrival-to-arrival time are higher in segments with large $\mathrm{RC}$ ratios. The mean values of the red variable indicate that the percentage of bus trips that encountered a red signal delay is also higher in segments with large RC ratios. Mean traffic volumes are higher in segments that are close to the west side of this corridor (close to the Ross Island Bridge). About $20 \%$ of the bus trips were made during peak hours at all segments. Departure delay, as an indicator of service reliability, varies significantly across segments. The percentage of bus trips that skipped the departure stop is higher at far-side segments, because the departure stop usually has fewer passengers than the arrival stop in a far-side segment. Passenger activities are higher at near-side segments because the departure stop of a nearside segment usually has more passengers.

\subsection{Empirical Results}

This section presents the results of the pooled and individual regression models. The regression models were estimated using the $\mathrm{R}$ statistical programming package stepwise regression function. An alpha level of 0.05 was used for the statistical tests. Only variables that are significant at the 0.05 level are retained in the final regression models. In each model, the estimated unstandardized coefficients, standard errors, t-value and Rsquare change $\left(\Delta \mathbf{R}^{2}\right)$ are reported. The R-square change was computed by squaring the 
semi-partial correlation coefficient of each variable estimated by the R package "ppcor" (Kim, 2013). It represents the change of R square by adding a variable to or removing a variable from a model. In other words, the R-square change is a way to represent the travel time variance explained by one variable, ceteris paribus.

\subsubsection{Far-side segments stop-to-stop travel time models}

Table 3-5 shows the results of the far-side segments departure-to-arrival time model and arrival-to-arrival time model. Variables without estimated coefficients are not significant at the 0.05 level; variables that are not estimated in the model are shown as "-". The total number of bus stop-to-stop trips for far-side segments is 34,070 . The far-side segments departure-to-arrival time model and the arrival-to-arrival time model explain $78 \%$ and $75 \%$ of the total travel time variation, respectively. Most of the estimated coefficients between the two models are very similar except for the skip variable, these results indicate that the estimated effects of signal delay and traffic conditions on bus travel time are robust.

All three travel impedance variables (distance, traffic volume and peak) are significant and positive. Each one mile increase in distance results in an average of 137 and 115 seconds increase in bus departure-to-arrival time and bus arrival-to-arrival time, respectively, controlling for other variables. This is equivalent to an average speed of 26 and $31 \mathrm{mph}$, respectively. For each 1,000 vehicles per hour increase in traffic volumes, bus travel time increases by 2 seconds. If a bus travels in the AM peak hours in the westbound direction or in the PM peak hours in the eastbound direction, the departure-to- 
arrival time and arrival-to-arrival time will increase an average of 2.4 and 2.7 seconds, respectively.

Table 3-5 Far-side segments stop-to-stop travel time models

\begin{tabular}{|c|c|c|c|c|c|c|c|c|}
\hline \multirow[t]{2}{*}{ Far-side segments } & \multicolumn{4}{|c|}{ Departure-to-arrival time } & \multicolumn{4}{|c|}{ Arrival-to-arrival time } \\
\hline & Coeff. & S.E. & t-value & $\Delta \mathbf{R}^{2}$ & Coeff. & S.E. & t-value & $\Delta \mathbf{R}^{2}$ \\
\hline (Intercept) & 1.6 & 0.4 & 4.5 & & 19.1 & 0.5 & 39.2 & \\
\hline Distance & 137 & 2 & 58 & 0.022 & 115 & 3 & 36 & 0.009 \\
\hline Volume (vph*1000) & 2.1 & 0.2 & 11.6 & 0.002 & 2.4 & 0.2 & 9.8 & 0.008 \\
\hline Peak & 2.4 & 0.2 & 14.4 & 0.013 & 2.7 & 0.2 & 11.8 & 0.015 \\
\hline Red time (sec) & 0.70 & 0.0 & 273.3 & 0.496 & 0.70 & 0.0 & 202.3 & 0.358 \\
\hline $\mathrm{RC}$ ratio & 14.2 & 0.4 & 37 & 0.245 & 17.5 & 0.5 & 33.6 & 0.193 \\
\hline Ons & - & - & - & - & 3.4 & 0.1 & 50.1 & 0.077 \\
\hline Offs & - & - & - & - & & & & \\
\hline Lift & - & - & - & - & 37.1 & 1.1 & 35.0 & 0.011 \\
\hline Depart delay (min) & -0.1 & 0.0 & -7.2 & 0.001 & -0.2 & 0.0 & -6.4 & 0 \\
\hline Skip & -2.8 & 0.1 & -24.7 & 0.003 & -18.1 & 0.2 & -106.4 & 0.079 \\
\hline R-Square & 0.78 & & & & 0.75 & & & \\
\hline Adj. R-Square & 0.78 & & & & 0.75 & & & \\
\hline $\mathrm{N}$ & 34070 & & & & 34070 & & & \\
\hline
\end{tabular}

Signal delay variables (red time and $R C$ ratio) are also significant and positive. Results indicate that if a bus trip experiences a red signal delay, the average signal delay will be equal to $70 \%$ of the red phase duration. Also, segments with $10 \%$ higher $\mathrm{RC}$ ratios have an average of 1.42 seconds (and 1.75 seconds) additional delay for the departure-toarrival time (and arrival-to-arrival time). For example, the average red phase durations for $39^{\text {th }} \mathrm{EB}$ and $65^{\text {th }} \mathrm{EB}$ are 66 seconds and 16 seconds, respectively, and the average cycle lengths are almost the same between the two segments (120 seconds). Therefore, the RC ratios at these two segments are 0.55 and 0.13 , respectively. The average values of red time variables are 28.3 seconds and 1.7 seconds, respectively. The average signal delays 
for $39^{\text {th }} \mathrm{EB}$ and $65^{\text {th }} \mathrm{EB}$ segments are 27.6 seconds $\left(0.55^{*} 14.2+28.3 * 70 \%\right)$ and 3.1 seconds $(0.13 * 14.2+1.7 * 70 \%)$, respectively. The red time and $\mathrm{RC}$ ratios variables have the largest R-square changes (74\% in the departure-to-arrival time model and $54 \%$ in the arrival-to-arrival time model), which indicates that most of the bus stop-to-stop travel time variation is due to signal delay controlling for other variables. The $\mathrm{RC}$ ratio coefficient is slightly higher in the arrival-to-arrival time model; this may be because the queuing effect in far-side segments may extend to the upstream stop of a stop-to-stop segment.

Passenger activity variables (ons, offs and lift) are only estimated in the arrival-to-arrival time model. The effects of passenger boarding and lift use are significant and positive. Each additional passenger boarding increases bus arrival-to-arrival time by 3.4 seconds and each additional lift use increases bus arrival-to-arrival time by 37.1 seconds. The effect of passenger alighting is not significant.

Departure delay and skip variables are both significant and negative. Results indicate that for each additional minute increase in departure stop schedule delay, bus travel time decreases by 0.1 seconds. If a bus skips the departure stop, bus departure-to-arrival time and arrival-to-arrival time decrease by 2.8 and 17.9 seconds, respectively. This is because stop skipping only saves partial acceleration delay for departure-to-arrival time, but it saves acceleration and deceleration delay, as well as dwell time for arrival-to-arrival time. 


\subsubsection{All segments arrival-to-arrival time model}

The results of the all segments arrival-to-arrival time model are presented in Table 3-6.

Table 3-6 All segments arrival-to-arrival time model

\begin{tabular}{l|rrrr}
\hline \multirow{2}{*}{ All segments } & \multicolumn{4}{|c}{ Arrival-to-arrival time } \\
\cline { 2 - 5 } & Coeff. & S.E. & t-value & $\Delta \mathbf{R}^{\mathbf{2}}$ \\
\hline (Intercept) & 18.3 & 0.5 & 40.4 & \\
Distance & 114.6 & 3.2 & 36.3 & 0.007 \\
Distance*near & 23.9 & 4.2 & 5.7 & 0.009 \\
Red time (sec) & 2.4 & 0.2 & 15.6 & 0.007 \\
Red time*near & & & & \\
RC ratio & 3.1 & 0.2 & 18.4 & 0.013 \\
RC ratio*near & & & & \\
Volume (vph*1000) & 0.71 & 0.00 & 207.2 & 0.258 \\
Volume*near & -0.03 & 0.01 & -4.8 & 0.093 \\
Peak & 17.7 & 0.5 & 34.7 & 0.162 \\
Skip & -12.1 & 1.9 & -6.4 & 0.006 \\
Ons & 3.6 & 0.1 & 54.7 & 0.057 \\
Ons*near & -1.8 & 0.1 & -22.6 & 0.034 \\
Offs & & & & \\
Offs*near & & & & \\
Lift & 37.1 & 1.0 & 35.6 & 0.008 \\
Lift*near & -12.3 & 1.7 & -7.4 & 0.005 \\
Near & -17.3 & 0.1 & -126.0 & 0.078 \\
\hline R-Square & 0.74 & & & \\
Adj. R-Square & 0.74 & & & \\
N & 51307 & & & \\
\hline
\end{tabular}

This model explains $74 \%$ of the bus travel time variation with 51,307 bus trip

observations. Because bus stop location type may affect other variables' effects on bus arrival-to-arrival time, interaction effects between the near dummy variable and other variables were tested. Results show that the interaction effects are all significant, which means that most of the effects are significantly different between near-side and far-side 
segments. For those variables that are interacted with the near dummy variable, the coefficients of original variables represent their effects for far-side segments; the coefficients of the interaction variables represent the difference of those effects between near-side and far-side segments. Results show that the coefficients of those original variables are the same as in the far-side segments arrival-to-arrival model, which indicates the estimated coefficients are robust.

The effects of distance and traffic volume are slightly different between near-side and far-side segments. For each mile increase in travel distance, bus travel time increases by 114 and 138 seconds for far-side and near-side segments, respectively. For each 1,000 vehicles per hour increase in traffic volumes, bus arrival-to-arrival time increases by 2.4 seconds. Peak hour effect is the same between near-side and far-side segments. If a bus travels in the peak hours, bus travel time increases by an average of 3.4 seconds.

The effects of signal delay are also different between near-side and far-side segments. The average signal delay for far-side and near-side segments are $71 \%$ and $68 \%$ of the red phase duration, respectively. The additional queuing delay increases by 1.77 and 0.56 seconds for each $10 \%$ increase in RC ratio for far-side and near-side segments, respectively. Results show that signal delay effects are lower at near-side segments. This may be because some buses serve passengers when waiting for a red signal at near-side stops, and thus the ratio between waiting time for a red signal and the red phase duration is smaller. Also, because near-side bus stops are usually very close to intersections, there is less additional queuing effect because buses are always in the front of a queue when 
waiting for a red signal. However, bus queuing delay before a bus arrives at a near-side bus stop is not captured in this model.

The effects of passenger boarding and lift use are also different between near-side and far-side segments. Each additional passenger boarding increases bus travel time by 3.6 and 1.8 seconds for far-side and near-side segments, respectively. Each additional lift use increases bus travel time by 37.1 and 24.8 seconds for far-side and near-side segments, respectively. These differences are also likely because some buses serve passengers when they are waiting for a red signal at a near-side stop. The skip stop effect is the same between the two segment types. If a bus skips a departure stop of a segment, bus travel time reduces by 17 seconds on average. Bus arrival-to-arrival time is 2.9 seconds less at near-side segments than at far-side segments.

\subsubsection{Individual segment models}

Individual segment stop-to-stop travel time models are estimated to gain more insights into the effects of signal delay and traffic volume on bus stop-to-stop travel time at each individual segment. Both departure-to-arrival time models and arrival-to-arrival time models are estimated for each individual far-side segment, and arrival-to-arrival model is

estimated at each individual near-side segment. Results are shown in Table 3-7, Table 3-8 and Table 3-9. 
Table 3-7 Individual far-side segment departure-to-arrival time models

\begin{tabular}{|c|c|c|c|c|c|c|c|c|c|c|c|c|}
\hline \multirow[t]{2}{*}{ Far-side segments } & \multicolumn{2}{|c|}{ 39th EB } & \multicolumn{2}{|c|}{ 50th EB } & \multicolumn{2}{|c|}{ 52nd EB } & \multicolumn{2}{|c|}{ 65th EB } & \multicolumn{2}{|c|}{ 69th EB } & \multicolumn{2}{|c|}{$71 \mathrm{st} \mathrm{EB}$} \\
\hline & Coeff. & $\mathrm{t}$ & Coeff. & $\mathrm{t}$ & Coeff. & $\mathrm{t}$ & Coeff. & $\mathrm{t}$ & Coeff. & $\mathrm{t}$ & Coeff. & $\mathrm{t}$ \\
\hline (Intercept) & 26.1 & 27.2 & 26.2 & 42.2 & 14.4 & 23.3 & 19.7 & 50.2 & 18.0 & 70.7 & 20.5 & 45.5 \\
\hline Red & 39.9 & 62.8 & 41.8 & 92.1 & 48.8 & 101.9 & 18.6 & 44.9 & 18.1 & 39.5 & 20.2 & 40.8 \\
\hline volume (vph*1000) & 10.2 & 10.7 & 5.1 & 7.5 & 8.3 & 9.9 & 3.2 & 7.3 & 1.7 & 7.1 & 3.6 & 7.4 \\
\hline peak & 24.5 & 6.6 & & & 2.7 & 4.1 & & & & & 9.2 & 3.7 \\
\hline peak*volume & -13.2 & -5.0 & & & & & 0.7 & 2.2 & & & -6.9 & -3.6 \\
\hline depart delay (min) & -0.2 & -2.2 & & & 0.1 & 2.1 & -0.1 & -2.9 & -0.1 & -3.7 & -0.1 & -3.0 \\
\hline skip & -1.9 & -3.0 & -1.6 & -3.6 & -2.1 & -4.5 & -2.5 & -9.7 & -2.4 & -13.6 & -3.0 & -10.1 \\
\hline \multirow{3}{*}{\multicolumn{13}{|c|}{$\begin{array}{l}\text { ons } \\
\text { offs } \\
\text { lift }\end{array}$}} \\
\hline & & & & & & & & & & & & \\
\hline & & & & & & & & & & & & \\
\hline \multirow{4}{*}{$\begin{array}{l}\text { R-Square } \\
\mathrm{N}\end{array}$} & \multirow{2}{*}{\multicolumn{2}{|c|}{$\begin{array}{r}0.62 \\
2935 \\
\end{array}$}} & \multirow{2}{*}{\multicolumn{2}{|c|}{$\begin{array}{r}0.76 \\
2934 \\
\end{array}$}} & \multirow{2}{*}{\multicolumn{2}{|c|}{$\begin{array}{r}0.82 \\
2895 \\
\end{array}$}} & \multirow{2}{*}{\multicolumn{2}{|c|}{$\begin{array}{r}0.46 \\
2919\end{array}$}} & \multirow{2}{*}{\multicolumn{2}{|c|}{$\begin{array}{r}0.4 \\
2932 \\
\end{array}$}} & \multirow{2}{*}{\multicolumn{2}{|c|}{$\begin{array}{r}0.42 \\
2930\end{array}$}} \\
\hline & & & & & & & & & & & & \\
\hline & \multicolumn{2}{|c|}{ 33rd WB } & \multicolumn{2}{|c|}{ 39th WB } & \multicolumn{2}{|c|}{ 50th WB } & \multicolumn{2}{|c|}{ 52nd WB } & \multicolumn{2}{|c|}{ 65th WB } & \multicolumn{2}{|c|}{ 72nd WB } \\
\hline & Coeff. & $\mathrm{t}$ & Coeff. & $\mathrm{t}$ & Coeff. & $\mathrm{t}$ & Coeff. & $\mathrm{t}$ & Coeff. & $\mathrm{t}$ & Coeff. & $\mathrm{t}$ \\
\hline (Intercept) & 19.8 & 36.0 & 31.3 & 32.0 & 20.4 & 26.3 & 27.3 & 37.4 & 16.1 & 50.0 & 27.7 & 57.3 \\
\hline Red & 22.4 & 44.9 & 45.7 & 77.3 & 49.9 & 96.1 & 31.6 & 59.3 & 16.7 & 46.0 & 20.3 & 40.7 \\
\hline volume (vph*1000) & 2.8 & 6.5 & -4.7 & -4.8 & 9.9 & 9.7 & 3.7 & 5.1 & 3.3 & 9.5 & 1.6 & 2.9 \\
\hline peak & 7.8 & 4.1 & 4.7 & 5.3 & & & 2.3 & 3.6 & & & 4.9 & 3.3 \\
\hline peak*volume & -3.6 & -2.8 & & & & & & & & & -3.8 & -2.5 \\
\hline depart delay (min) & & & -0.3 & -2.5 & & & -0.5 & -4.7 & & & -0.4 & -5.0 \\
\hline skip & -1.3 & -4.2 & -3.4 & -5.6 & & & -3.0 & -6.2 & -2.3 & -11.6 & -2.3 & -7.6 \\
\hline \multicolumn{13}{|l|}{ ons } \\
\hline \multirow{2}{*}{\multicolumn{13}{|c|}{$\begin{array}{l}\text { offs } \\
\text { lift }\end{array}$}} \\
\hline & & & & & & & & & & & & \\
\hline R-Square & 0.46 & & 0.69 & & 0.78 & & 0.59 & & 0.48 & & 0.41 & \\
\hline $\mathrm{N}$ & 2756 & & 2758 & & 2761 & & 2741 & & 2759 & & 2760 & \\
\hline
\end{tabular}


Table 3-8 Individual far-side segment arrival-to-arrival time models

\begin{tabular}{|c|c|c|c|c|c|c|c|c|c|c|c|c|}
\hline \multirow{2}{*}{ Far-side segments } & \multicolumn{2}{|c|}{ 39th EB } & \multicolumn{2}{|c|}{ 50th EB } & \multicolumn{2}{|c|}{ 52nd EB } & \multicolumn{2}{|c|}{65 th EB } & \multicolumn{2}{|c|}{ 69th EB } & \multicolumn{2}{|c|}{$71 \mathrm{st}$ EB } \\
\hline & Coeff. & $\mathrm{t}$ & Coeff. & $\mathrm{t}$ & Coeff. & $\mathrm{t}$ & Coeff. & $\mathrm{t}$ & Coeff. & $\mathrm{t}$ & Coeff. & $\mathrm{t}$ \\
\hline (Intercept) & 39.5 & 39.1 & 38.8 & 49.0 & 29.2 & 31.9 & 33.2 & 76.7 & 32.3 & 72.0 & 35.2 & 59.1 \\
\hline Red & 39.9 & 60.0 & 41.7 & 83.3 & 50.6 & 69.1 & 18.5 & 38.2 & 17.7 & 23.3 & 20.0 & 31.7 \\
\hline volume $\left(\mathrm{vph}^{*} 1000\right)$ & 11.3 & 11.2 & 8.5 & 8.3 & 14.4 & 11.2 & 3.5 & 8.8 & 2.2 & 5.5 & 4.4 & 7.2 \\
\hline peak & 25.2 & 6.5 & & & 2.8 & 2.8 & & & & & 12.4 & 3.9 \\
\hline peak*volume & -13.6 & -4.9 & -2.5 & -3.4 & & & & & & & -9.4 & -3.9 \\
\hline depart delay (min) & & & & & & & & & -0.1 & -3.0 & -0.2 & -3.6 \\
\hline skip & -16.2 & -22.9 & -15.3 & -28.6 & -19.2 & -25.9 & -15.9 & -50.0 & -16.8 & -52.3 & -18.1 & -43.2 \\
\hline ons & 1.9 & 4.4 & 2.7 & 10.0 & 2.3 & 9.8 & 3.6 & 9.5 & 3.5 & 13.1 & 4.6 & 11.6 \\
\hline offs & -0.1 & -2.4 & & & & & 0.0 & -3.1 & & & & \\
\hline lift & & & 36.4 & 12.8 & 40.8 & 13.5 & & & 24.3 & 7.0 & 33.1 & 16.6 \\
\hline \multirow{4}{*}{$\begin{array}{l}\text { R-Square } \\
\mathrm{N}\end{array}$} & 0.64 & & 0.77 & & 0.76 & & 0.66 & & 0.64 & & 0.64 & \\
\hline & 2935 & & 2934 & & 2895 & & 2919 & & 2932 & & 2930 & \\
\hline & 33rd & WB & 39th & $\mathrm{WB}$ & 50th & $\overline{\mathrm{WB}}$ & $52 \mathrm{nc}$ & WB & 65 th & WB & $72 \mathrm{nd}$ & WB \\
\hline & Coeff. & $\mathrm{t}$ & Coeff. & $\mathrm{t}$ & Coeff. & $\mathrm{t}$ & Coeff. & $\mathrm{t}$ & Coeff. & $\mathrm{t}$ & Coeff. & $\mathrm{t}$ \\
\hline (Intercept) & 31.0 & 35.5 & 44.9 & 41.7 & 42.6 & 23.3 & 40.2 & 45.9 & 30.0 & 38.7 & 40.4 & 62.2 \\
\hline Red & 22.4 & 29.8 & 46.0 & 72.5 & 47.9 & 46.5 & 31.2 & 50.8 & 17.2 & 23.9 & 19.9 & 32.6 \\
\hline volume (vph*1000) & 5.1 & 7.8 & -4.3 & -4.0 & 14.7 & 5.7 & 4.7 & 5.7 & 5.5 & 6.2 & 2.7 & 4.0 \\
\hline peak & 11.6 & 4.0 & 4.0 & 4.3 & 49.0 & 5.3 & 3.1 & 4.2 & 7.1 & 2.5 & 6.1 & 3.3 \\
\hline peak*volume & -7.9 & -4.1 & & & -43.1 & -4.7 & & & -6.1 & -2.4 & -5.2 & -2.9 \\
\hline depart delay (min) & 0.6 & 6.1 & & & -0.9 & -3.8 & -0.3 & -2.7 & 0.2 & 2.3 & -0.3 & -3.0 \\
\hline skip & -14.2 & -26.1 & -16.9 & -24.0 & -23.0 & -15.0 & -16.1 & -25.7 & -17.2 & -36.2 & -15.4 & -32.9 \\
\hline ons & 3.6 & 16.7 & 3.5 & 7.3 & 2.4 & 11.6 & 3.6 & 12.6 & 3.1 & 13.4 & 3.1 & 11.0 \\
\hline offs & 0.0 & 2.8 & & & & & & & & & & \\
\hline lift & 49.9 & 23.5 & 17.6 & 2.4 & 30.6 & 7.3 & 110.4 & 8.1 & 25.6 & 3.7 & 34.7 & 16.0 \\
\hline $\begin{array}{l}\text { R-Square } \\
\mathrm{N}\end{array}$ & $\begin{array}{r}0.62 \\
2756\end{array}$ & & $\begin{array}{r}0.71 \\
2758\end{array}$ & & $\begin{array}{r}0.57 \\
2761\end{array}$ & & $\begin{array}{r}0.66 \\
2741\end{array}$ & & $\begin{array}{r}0.6 \\
2759\end{array}$ & & $\begin{array}{r}0.62 \\
2760\end{array}$ & \\
\hline
\end{tabular}


Table 3-9 Individual near-side segment arrival-to-arrival time models

\begin{tabular}{l|rr|rr|rr|rr|rr|rr|rr}
\hline \multirow{2}{*}{ near-side } & \multicolumn{2}{|c|}{ 26th EB } & \multicolumn{2}{|c|}{ 26th WB } & \multicolumn{2}{|c|}{ 33rd EB } & \multicolumn{2}{|c|}{ 42nd EB } & \multicolumn{2}{c|}{ 42nd WB } & \multicolumn{2}{c}{72 nd EB } \\
\cline { 2 - 12 } & Coeff. & $\mathrm{t}$ & Coeff. & $\mathrm{t}$ & Coeff. & $\mathrm{t}$ & Coeff. & $\mathrm{t}$ & Coeff. & $\mathrm{t}$ & Coeff. & $\mathrm{t}$ \\
\hline (Intercept) & 42.7 & 36.8 & 33.3 & 71.9 & 28.8 & 46.2 & 48.2 & 105.0 & 33.8 & 73.9 & 37.8 & 62.4 \\
Red & 27.4 & 33.0 & 26.1 & 39.8 & 16.4 & 34.6 & 20.2 & 39.3 & 20.8 & 42.6 & 13.7 & 26.4 \\
volume (vph*1000) & 7.6 & 6.8 & & & 4.6 & 8.9 & & & & & 5.0 & 6.8 \\
peak & 3.6 & 2.8 & 60.4 & 14.0 & 23.5 & 4.3 & 5.2 & 9.3 & 5.2 & 10.6 & 15.1 & 6.2 \\
peak*volume & & & -33.3 & -12.2 & -10.9 & -3.5 & & & & & -11.7 & -5.6 \\
depart delay (min) & & & & & & & & & & & \\
skip & -20.4 & -18.6 & -14.2 & -18.8 & -13.0 & -29.8 & -16.0 & -31.5 & -15.5 & -30.4 & -17.8 & -39.2 \\
ons & 1.8 & 15.7 & 2.4 & 33.6 & 3.2 & 13.8 & 3.0 & 8.7 & 2.0 & 8.5 & 2.5 & 9.7 \\
offs & & & 0.1 & 3.8 & & & 0.0 & -2.4 & 0.1 & 3.3 & 0.0 & -2.0 \\
lift & 19.2 & 4.3 & 26.4 & 4.3 & 31.5 & 10.2 & 40.5 & 4.8 & 26.3 & 2.7 & 28.8 & 22.2 \\
\hline R-Square & 0.56 & & 0.67 & & 0.62 & & 0.63 & & 0.74 & & 0.64 & \\
Adj. R-Square & 0.56 & & 0.67 & & 0.62 & & 0.63 & & 0.74 & & 0.64 \\
N & 2930 & & 2754 & & 2927 & & 2936 & & 2757 & & 2933 \\
\hline
\end{tabular}


The far-side segments departure-to-arrival time models explain $40-82 \%$ of the travel time variation. The far-side segments arrival-to-arrival time models explain 57-77\% of the travel time variation. Segments with high RC ratios have slightly higher R-square values in the departure-to-arrival time models than in the arrival-to-arrival time models; however, segments with low RC ratios have higher R-square values in the arrival-toarrival time models than in the departure-to-arrival models. This may be because the proportion of bus stop-to-stop travel time variation explained by signal delay is higher along segments with large RC ratios. Near-side segments arrival-to-arrival time models explain $56 \%-74 \%$ of total travel time variation.

The red dummy variable in these individual segment models is used to assess the average signal delay if a bus trip encounters a red signal. The coefficients of this variable are significant and positive for each segment. These coefficients are robust because they are almost the same between the departure-to-arrival time models and the arrival-to-arrival time models for far-side segments. The values of these coefficients are linearly related to the average red phase durations (or RC ratio). Figure 3-2 shows the relationship between the estimated coefficients of the red dummy variables and the average red phase durations at all stop-to-stop segments. It shows a clear linear relationship between the estimated red dummy variable coefficients and the average red phase durations. The slopes of the three lines are the same (0.6), which indicates that the waiting time for the red signal component of the signal delay is $60 \%$ of the red phase duration. The deceleration/acceleration delay and queuing effect delay components of the signal delay are included in the intercepts of the fitted lines. The intercepts indicate that the sum of 
average deceleration/acceleration delay and queuing effect delay at far-side segments is 12.1 seconds, and the average queuing effect delay at near-side segments is 3.9 seconds. This difference between near-side and far-side segments is likely because buses that encountered a red signal delay in a far-side segment had additional acceleration and deceleration delays compared to buses that did not encounter a red signal delay; while buses at near-side segments did not have additional deceleration/acceleration delay whether they encountered a red signal delay or not.

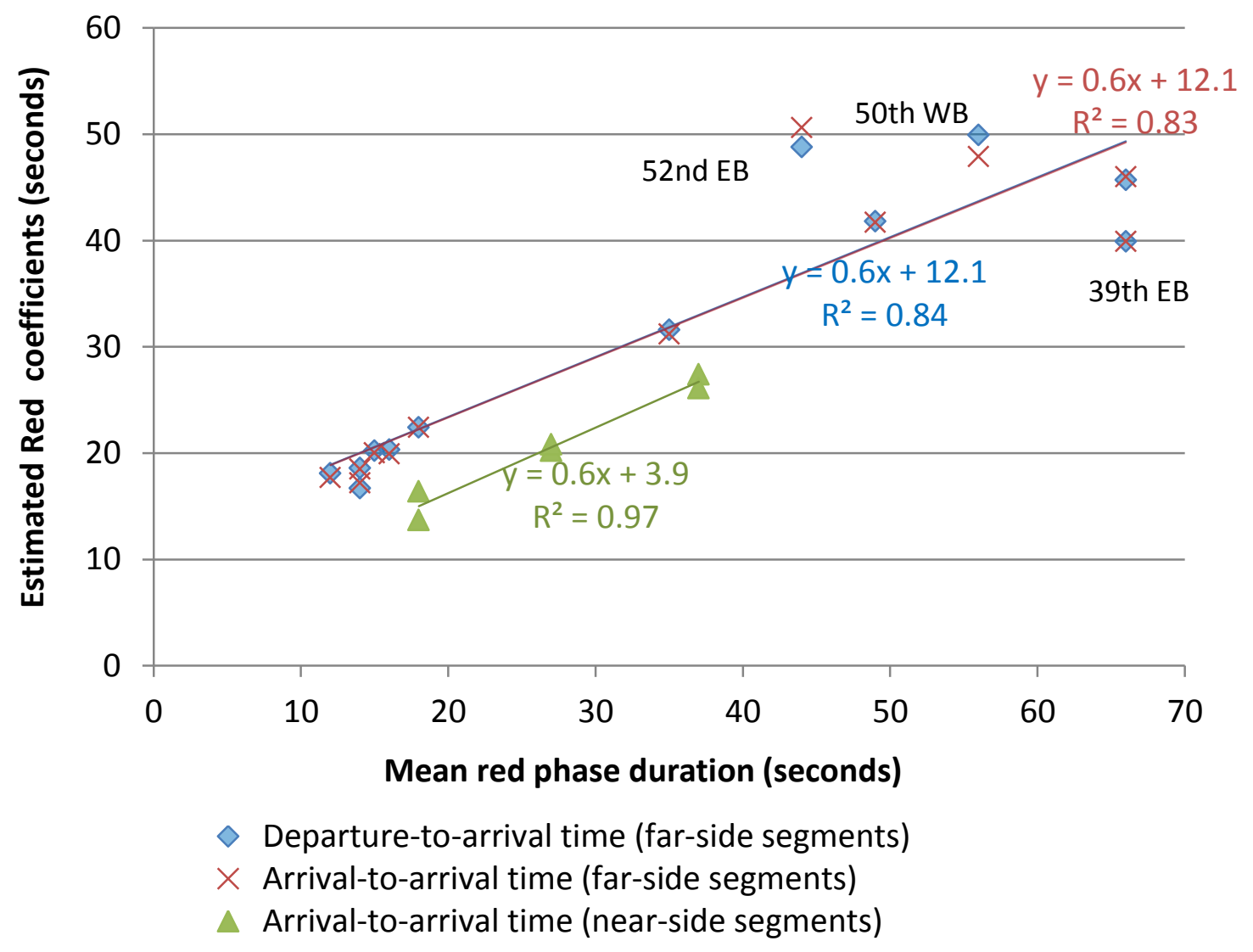

Figure 3-2 Red dummy variable coefficients vs. median red phase durations 
However, some estimated red variable coefficients are far from the fitted line. For example, the average red phase delay at $52^{\text {nd }} \mathrm{EB}$ and $50^{\text {th }} \mathrm{WB}$ are much higher than the predicted red phase delay by the fitted line. These deviations may be due to the additional queuing delay at these two segments. These two segments have high $\mathrm{RC}$ ratios and only two approaching lanes exist (no left turn lane) and all other segments with high RC ratios have at least three approaching lanes; therefore, buses have higher probabilities of waiting in a long queue at these two segments than at other segments. Conversely, although $39^{\text {th }}$ EB has large RC ratio, there are three through lanes and a left turn lane. All other segments with high $\mathrm{RC}$ ratios have at most two through lanes. Therefore, buses have higher probabilities of waiting in a short queue at this segment than at other segments.

The pooled regression models showed the average effects of traffic volumes on bus travel time. The individual regression models show that the effects of traffic volume vary significantly across segments and between peak and off-peak hours. The effects of traffic volume and peak are either positive or insignificant, and these effects are higher at segments with high $\mathrm{RC}$ ratios. The coefficients of traffic volume represent the effect of traffic conditions during off-peak hours. Results of the interaction effects between traffic volume and peak variables show that the effects of traffic volume during peak hours are significant and negative in most segments. This indicates that traffic congestion might occur at those segments during peak hours. The effects of passenger activities, departure delay and stop skipping vary slightly across segments. 


\subsection{Summary}

In summary, this chapter investigated factors that affect bus stop-to-stop travel time variability across intersections. Both the departure-to arrival time and the arrival-toarrival time models were estimated for far-side segments to analyze the impacts of intersection signal delay and traffic conditions on bus travel time and to validate the robustness of estimation results. The arrival-to-arrival time model was also estimated for all stop-to-stop segments to examine the effect of bus stop location type on bus travel time reliability. Finally, bus stop-to-stop travel time regression models were estimated at each individual segment to test whether the impacts of intersection signal delay and traffic conditions on bus travel time vary with intersection signal timing and geometric characteristics. Results indicate that intersection signal delay is the key source of bus stop-to-stop travel time variability across intersections. The effect of intersection signal delay is linearly related to intersection signal timing characteristics and significantly affected by intersection geometric characteristics. The effect of traffic conditions on bus travel time reliability is also affected by intersection signal timing and geometric characteristics. The effects of intersection delay and traffic conditions are also significantly different between near-side and far-side segments. Since intersection signal delay is the key source of bus stop-to-stop travel time delay and TSP is a strategy to reduce bus travel time delay through intersections, it is important to evaluate the TSP system performance. Because regression models have limited capabilities of assessing detailed performance of TSP systems, several new and useful performance measures are computed and presented in the next chapter. 


\section{TRANSIT SIGNAL PRIORITY PERFORMANCE EVALUATION}

To understand how well the transit signal priority (TSP) system helps buses reduce signal delay through signalized intersections, this chapter evaluates the relationships between TSP requests and TSP phases, the effectiveness of TSP phases (percent of effective TSP phases) and the expected benefits (time savings) and delay due to TSP phases. This chapter also investigates how these results vary by TSP phase type (green extension or early green), by bus stop location type (near-side or far-side bus stop) and by user type (bus, passengers, automobiles on the major street and the side street). TSP performance evaluation is based on the integration of two archived data sources: TriMet bus AVL/APC data and SCATS signal phase log data. Results from the evaluation analyses are useful for cities and transit agencies to identify potential problems, challenges and improvement opportunities.

\subsection{TSP System in Portland}

The current TSP system in Portland, Oregon is an active conditional TSP system. It consists of two systems: the TSP request system and the TSP phase grant system. If a bus meets certain conditions, an emitter on the bus will be activated. This emitter will send a TSP request to a downstream signalized intersection. Once the downstream traffic signal receives the TSP request, the signal controller in the traffic signal will decide whether to 
grant a TSP phase and which TSP phase to be granted. The decision frameworks of the two systems are shown in Figure 4-1 (a) and (b).

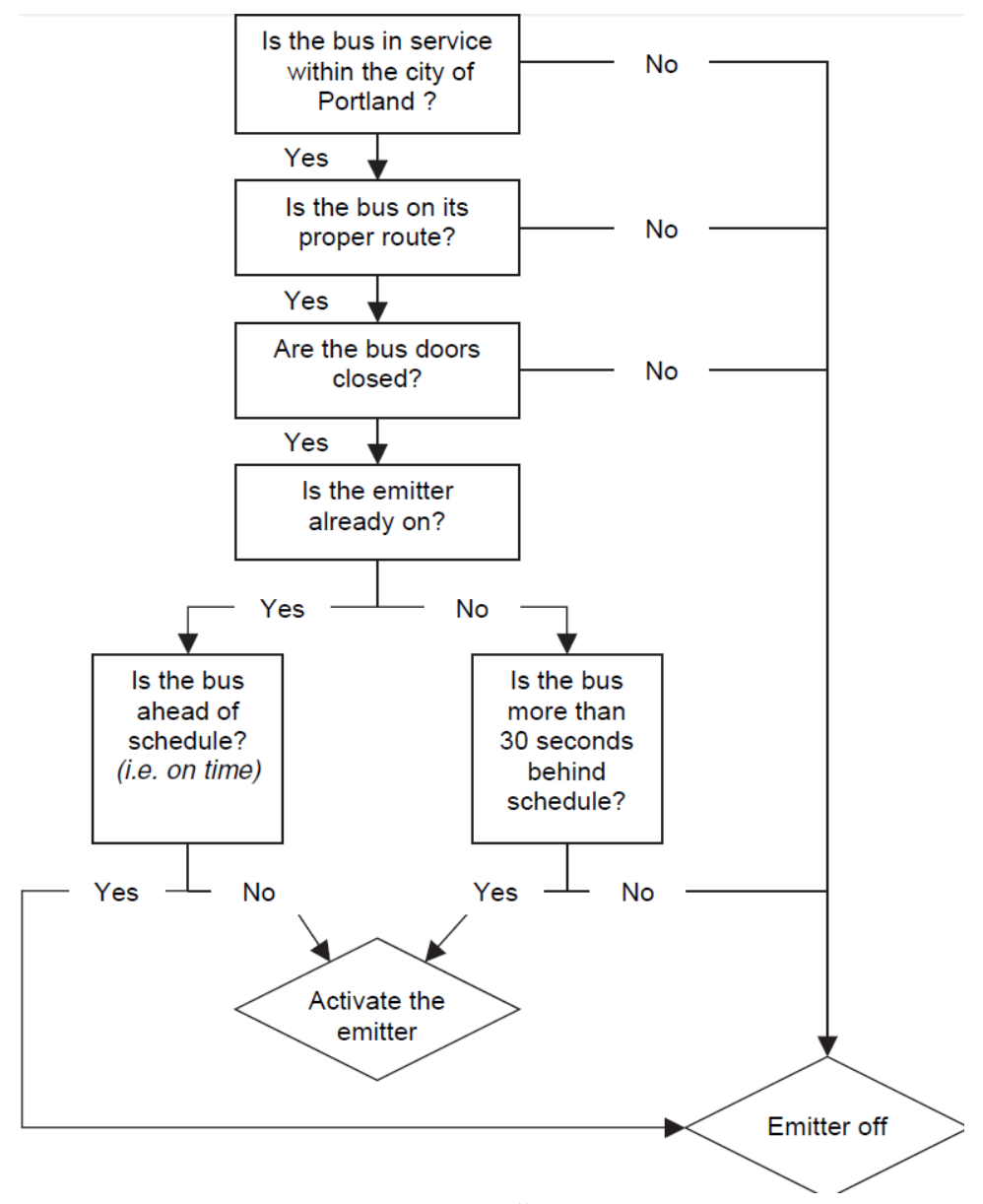

(a) TSP request

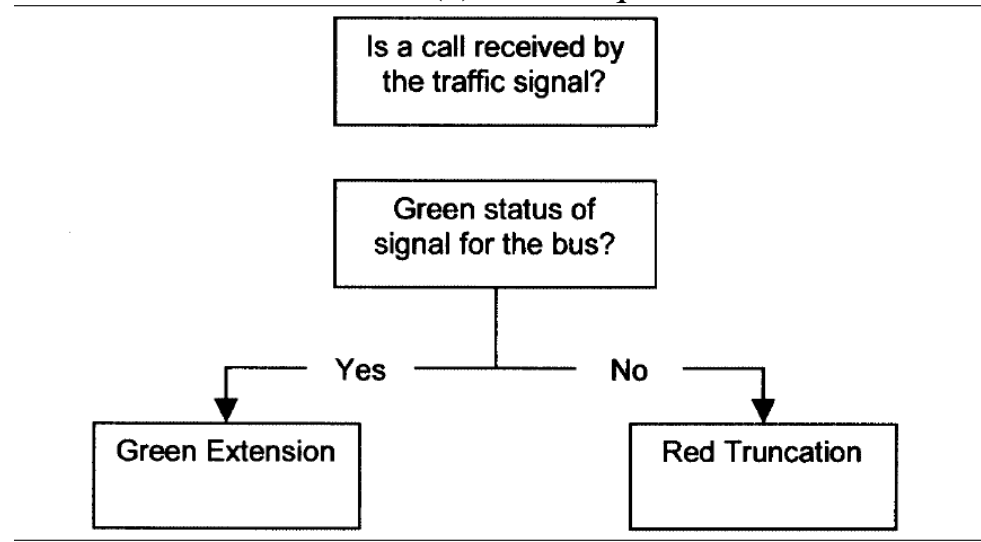

(b) TSP phase grant

Figure 4-1 TSP system decision framework (Byrne et al., 2005) 
Figure 4-2 shows the priority request detection zone length for each intersection from each direction.

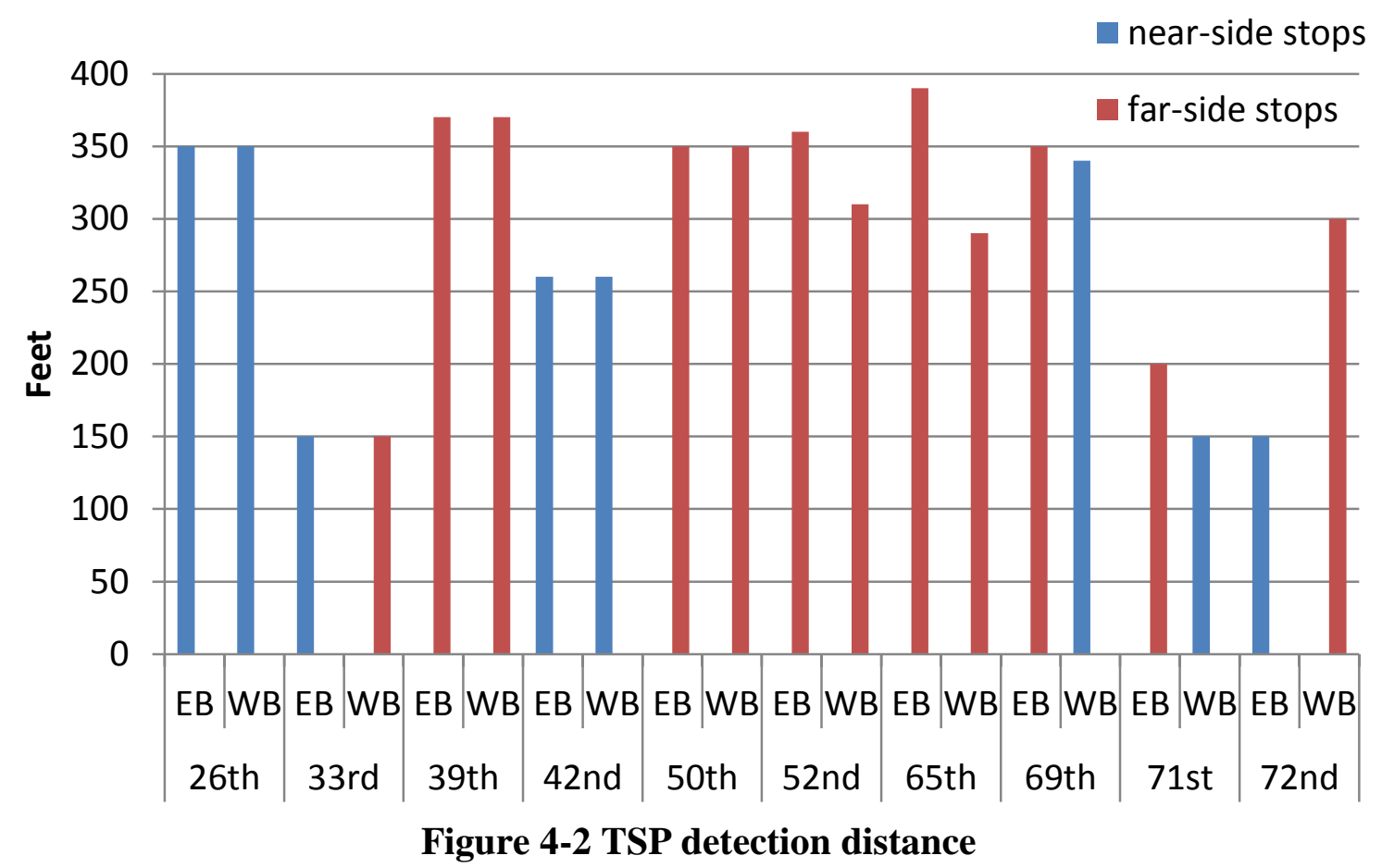

Figure 4-3 shows the average number of bus trips per day that requested TSP and that did not request TSP from both directions at intersections between $26^{\text {th }}$ Ave. and $72^{\text {nd }}$ Ave. along Powell Blvd. It shows that almost half of the bus trips requested TSP at each intersection. Figure 4-4 shows the average numbers of green extension phases and early green phases per day and the percentages of cycles that have a TSP phase. It shows that few TSP phases were granted at the intersections of $26^{\text {th }}$ Ave. and $33^{\text {rd }}$ Ave. on Powell Blvd., which indicates a potential TSP setting problem at these two intersections. The average duration of green extension phases is 7 seconds, and the average duration of early green phases is 11 seconds. Figure 4-3 and Figure 4-4 show that the average 
number of TSP requests is much higher than the number of TSP phases at each intersection. Therefore, not all of the TSP requests resulted in the granting of a TSP phase.
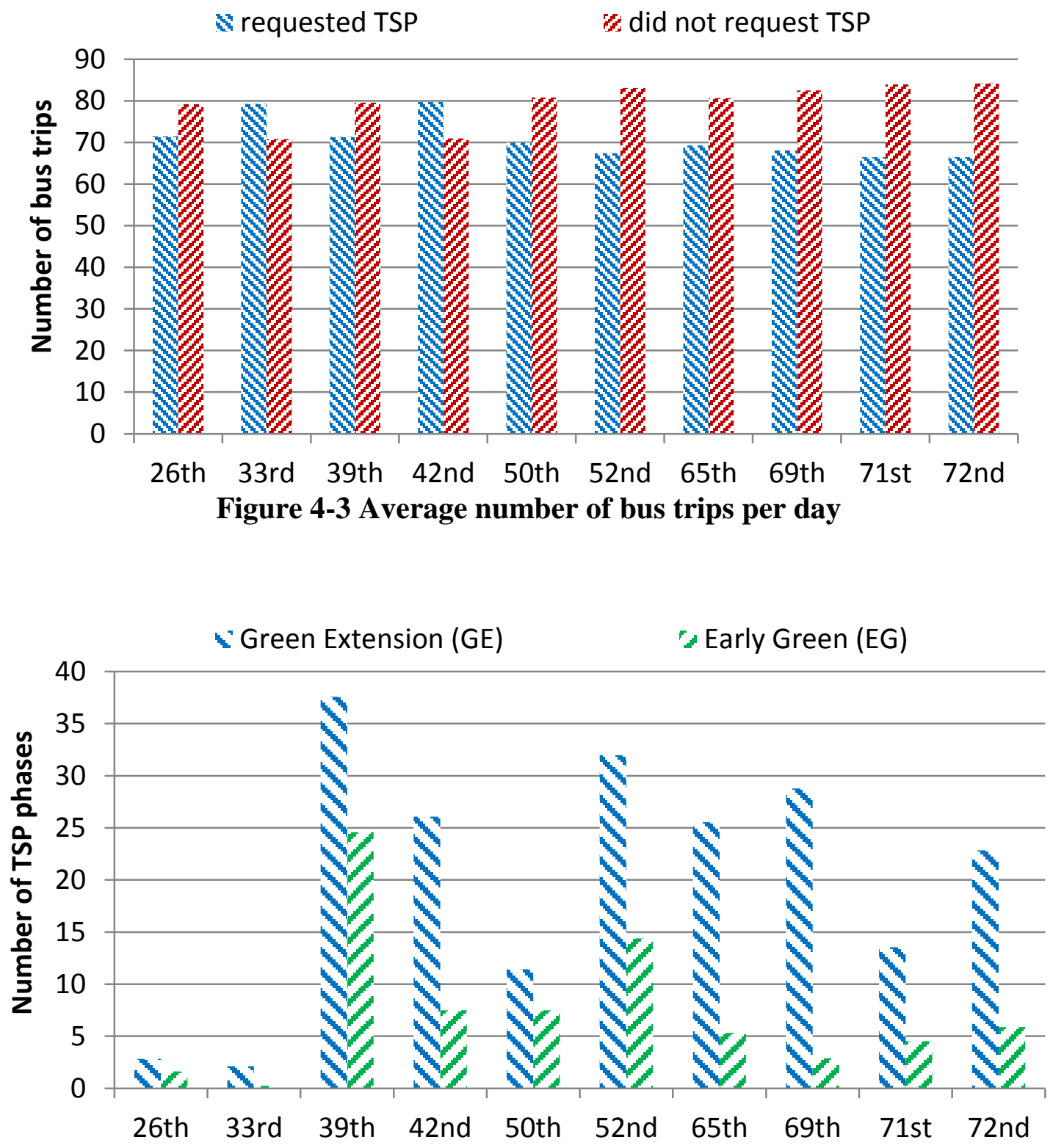

Figure 4-4 Average number of TSP phases per day 


\subsection{Relationships between TSP Phases and TSP Requests}

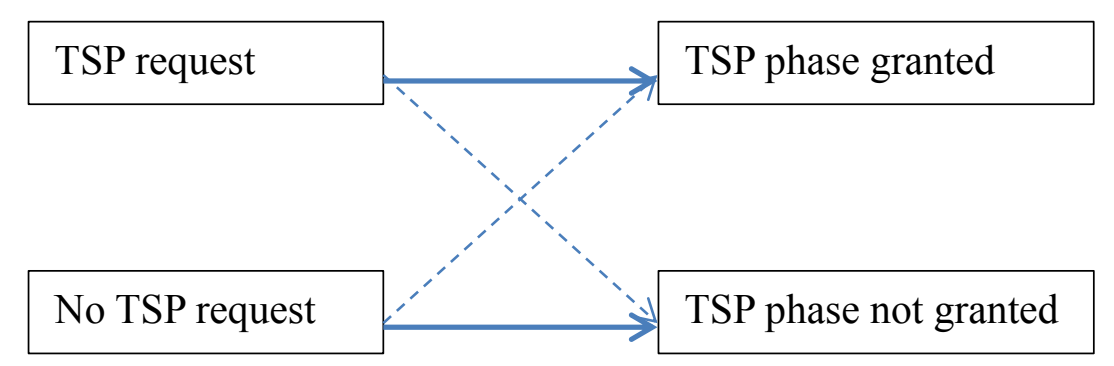

Figure 4-5 Relationships between TSP requests and TSP phases

Figure 4-5 shows the potential relationships between TSP requests and TSP phases. A TSP request may or may not result in the granting of a TSP phase. Also, a TSP phase may or may not result from a TSP request. Therefore, this section tries to answer the following two questions:

1) How many TSP requests resulted in the granting of a TSP phase?

2) How many TSP phases were a result of a TSP request?

\subsubsection{Assumptions and definitions}

To answer these two questions, some assumptions and definitions need to be clarified.

First, because there are no records of bus emitter activation or deactivation, once a bus meets the TSP request conditions shown in Figure 4-1 (a), it is assumed that the bus sends a TSP request to the downstream traffic signal. Second, it is important to define whether a TSP request triggered a TSP phase and whether a TSP phase was a result of a 
TSP request. For example, it is unreasonable to define that a bus TSP request resulted in a TSP phase granted if the TSP phase was granted 10 minutes after the TSP request. In this study, we define that a TSP request resulted in a TSP phase or a TSP phase was a result of a TSP request if the arrival time of the bus that requested TSP at an intersection and the start and end times of the TSP phase are close enough in time. In this study, we define that if the arrival time of a bus at the intersection and the TSP phase start time and end times are within the same cycle, then the bus that requests TSP is defined to have resulted in the granting of the TSP phase, and the TSP phase is defined to be a result of the TSP request from this bus.

\section{Green extension (GE)}

In order to define a cycle that includes the closest TSP requests to a GE phase, the cycle for GE analysis is defined as the time interval between the beginning times of two consecutive green phases. In other words, if the arrival time of a bus that requests TSP at an intersection is in a GE phase, in the preceding green phase of the GE phase, or the following red phase of the GE phase, this TSP request is defined to have resulted in the granting of the GE phase, or the GE phase is defined to be a result of the TSP request from this bus. Note that this cycle is for TSP performance analysis; it is different from the cycle definition in Chapter 2, which is used for data integration. Figure 4-6 shows the relationships between TSP requests and GE phases in six cycles. Because the bus arrival time at an intersection is probabilistic, a TSP request is defined as resulting in a GE phase, or a GE phase is defined as resulting from a TSP request, if the bus that requests TSP has 
a positive probability of arriving at an intersection during the GE phase, or the preceding green or the following red phase, for example, cycle (2) in Figure 4-6. Cycles (1) and (5) show examples of TSP requests that do not result in the granting of a GE phase. Cycle (3) shows an example of no TSP request (either no bus or a bus does not request TSP) and no granting of a GE phase. Cycles (4) and (6) show examples of granted GE phases without any TSP request in a cycle.
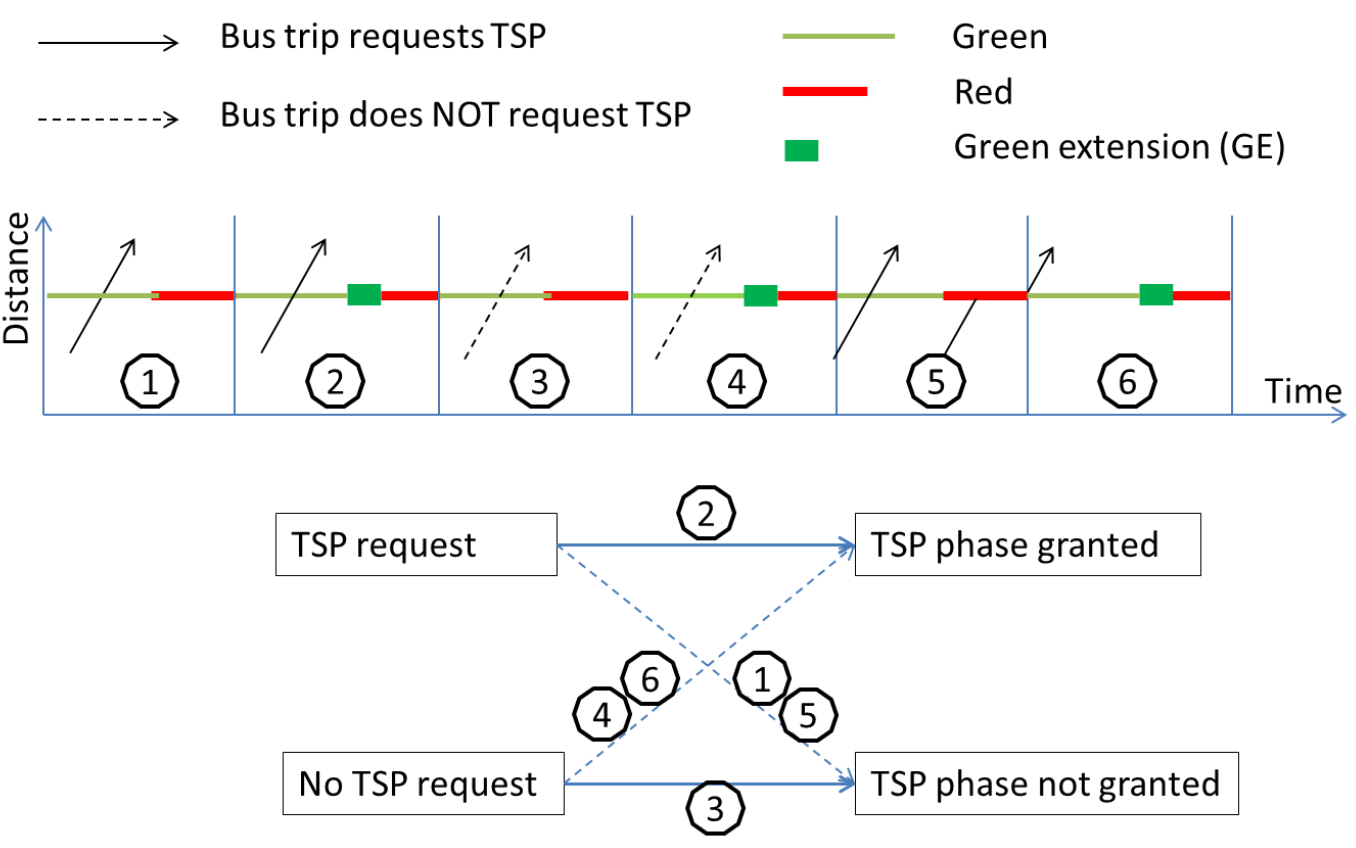

Figure 4-6 Relationships between TSP requests and green extension phases

2. Early green (EG)

Similarly, In order to define a cycle that includes the closest TSP requests to an EG phase, the cycle for EG analysis is defined as the time interval between the middle of two 
consecutive green phases. In other words, if the arrival time of a bus that requests TSP at an intersection is in an EG phase, in the preceding red phase of the EG phase, in the second half of the preceding green phase of the EG phase, or in the first half of the following green phase of the EG phase, this TSP request is defined to have resulted in the granting of the EG phase, or the EG phase is defined to be a result of the TSP request from this bus. Figure 4-7 shows the relationships between TSP requests and EG phases in six cycles. A TSP request is defined as resulting in an EG phase, or an EG phase is defined as resulting from a TSP request, if a bus that requests TSP has a positive probability of arriving at an intersection during the EG phase, the preceding red phase, the second half of the preceding green phase, or the first half of the following green phase, e.g. cycle (2) in Figure 4-7.
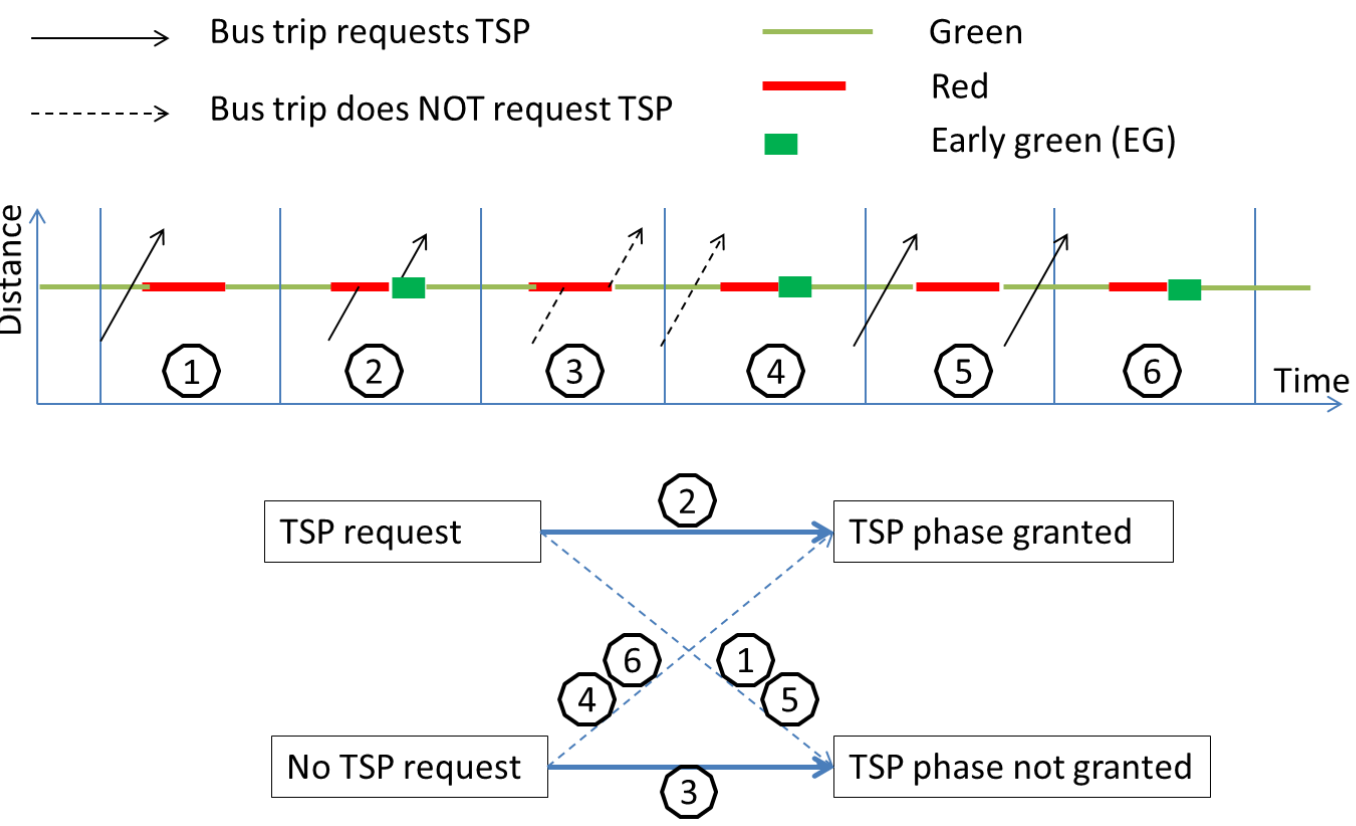

Figure 4-7 Relationships between TSP requests and early green phases 


\subsubsection{Results}

1. How many TSP requests resulted in TSP phases granted?

A TSP request could result in four potential outcomes: a GE phase was granted, an EG phase was granted, both GE and EG phases were granted, or no TSP phase was granted within a cycle. Results are shown in Figure 4-8. Because bus arrival time at an intersection is probabilistic, the four potential outcomes are described as below:

1) “GE only” means a bus that requested TSP had a positive probability of arriving at an intersection during a cycle with a GE phase but had zero probability of arriving at the intersection during a cycle with an EG phase.

2) “EG only" means a bus that requested TSP had a positive probability of arriving at an intersection during a cycle with an EG phase but had zero probability of arriving at the intersection during a cycle with a GE phase.

3) "Both $G E$ and $E G$ " means a bus that requested TSP had a positive probability of arriving at an intersection during a cycle with a GE phase and had a positive probability of arriving at the intersection during a cycle with an EG phase.

4) “Neither GE nor EG” means a bus that requested TSP had zero probability of arriving at an intersection during a cycle with a GE phase or during a cycle with an EG phase. 


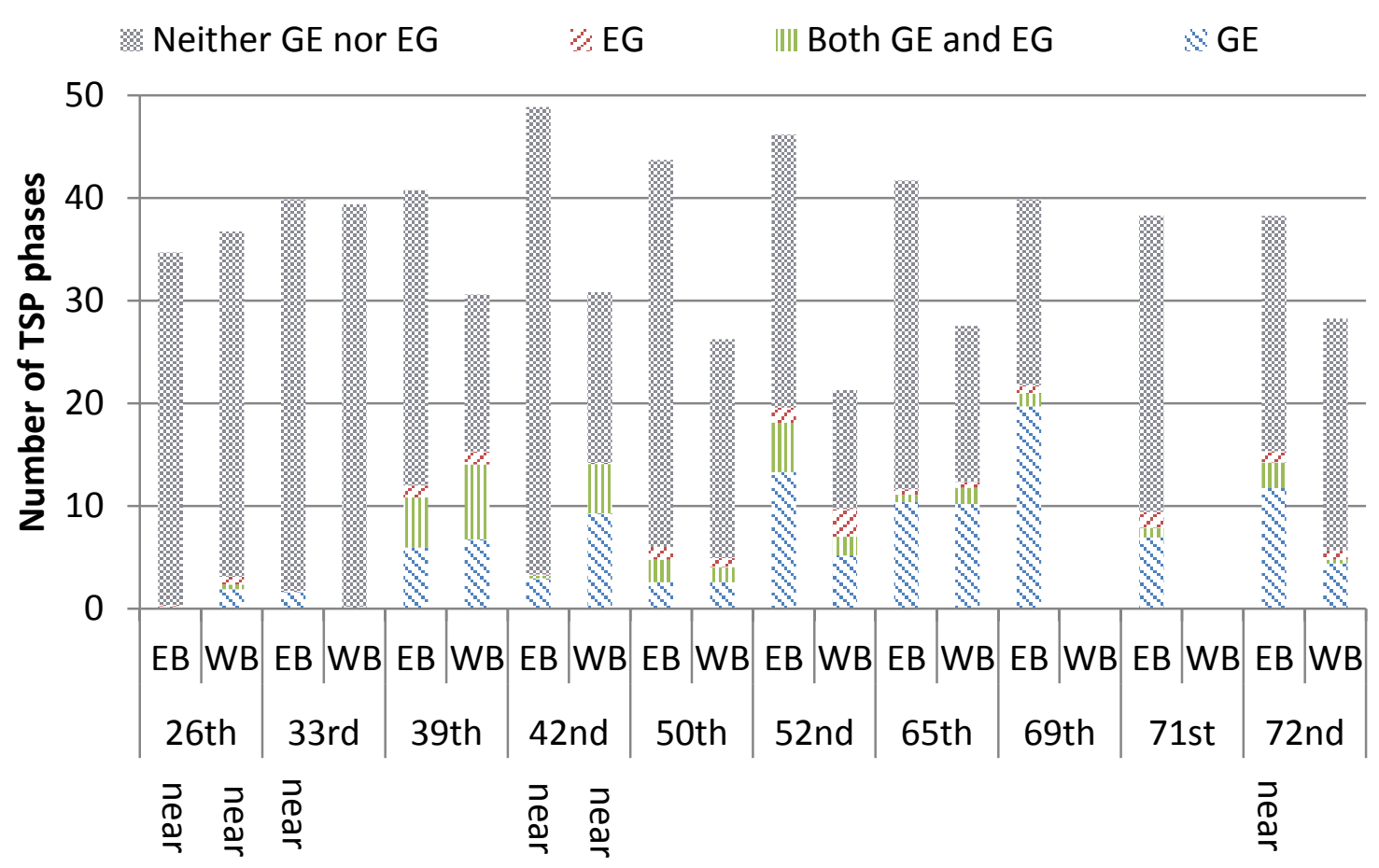

Figure 4-8 Average number of TSP requests per day

Figure 4-8 shows the breakdown of the average number of TSP requests per day at each intersection in each direction. Note that there are no results for the intersections at $69^{\text {th }}$ and $71^{\text {st }}$ Ave. on Powell Blvd. in the WB direction, because there are two signalized intersections in this stop-to-stop segment and bus arrival time at intersections are not available. Results vary significantly across intersections and by direction. For example, very few TSP requests resulted in the granting of a TSP phase at $42^{\text {nd }}$ Ave. in the EB direction, or at $26^{\text {th }}, 33^{\text {rd }}$ or $50^{\text {th }}$ Ave. in either direction. This indicates potential problems at these intersections. Results show that more than half of the TSP requests did not result in the granting of any TSP phases in most of the intersections in both directions. This may be because a bus emitter was not activated when conditions were met, a TSP request 
was not received by the traffic signal, or the signal controller did not decide to grant a TSP phase. Another interesting finding is that TSP requests resulted in more GE phases than EG phases. This may be because there is more green time in the EB and WB directions and buses have a higher probability of arriving at the intersection during a green phase. Also, there is no clear difference in the results between near-side segments and far-side segments in the plots.

2. How many granted TSP phases were a result of a TSP request?

A granted TSP phase could be a result of one or more TSP requests from EB only, from WB only or from both directions. It could also occur with no TSP requests from either direction in the same cycle. The granted TSP phases are broken down into the following four categories:

1) “EB only" means at least one EB bus that requested TSP had a positive probability of arriving at the intersection during the cycle with this TSP phase and no WB bus had a positive probability of arriving at the intersection during the same cycle.

2) “WB only" means at least one WB bus that requested TSP had a positive probability of arriving at the intersection during the cycle with this TSP phase and no EB bus had a positive probability of arriving at the intersection during the same cycle.

3) "Both $E B$ and $W B$ " means at least one EB bus and one WB bus that requested TSP had a positive probability of arriving at the intersection during the cycle with this TSP phase. 
4) “Neither EB nor WB" means no EB or WB bus that requested TSP had a positive probability of arriving at the intersection during the cycle with this TSP phase.

Figure 4-9 shows the breakdown of the average number of granted GE and EG phases for each intersection. There are more GE phases than EG phases, $90 \%$ of the GE and EG phases resulted from TSP requests within a cycle. However, about $30 \%$ of GE and EG phases at $39^{\text {th }}$ Ave. and $30 \%$ of GE phases at $42^{\text {nd }}$ Ave. did not result from a TSP request in the same cycle. This may indicate problems with TSP request detection or deactivation at these intersections. For example, a TSP call in the signal controller was not canceled after a bus passed the intersection.

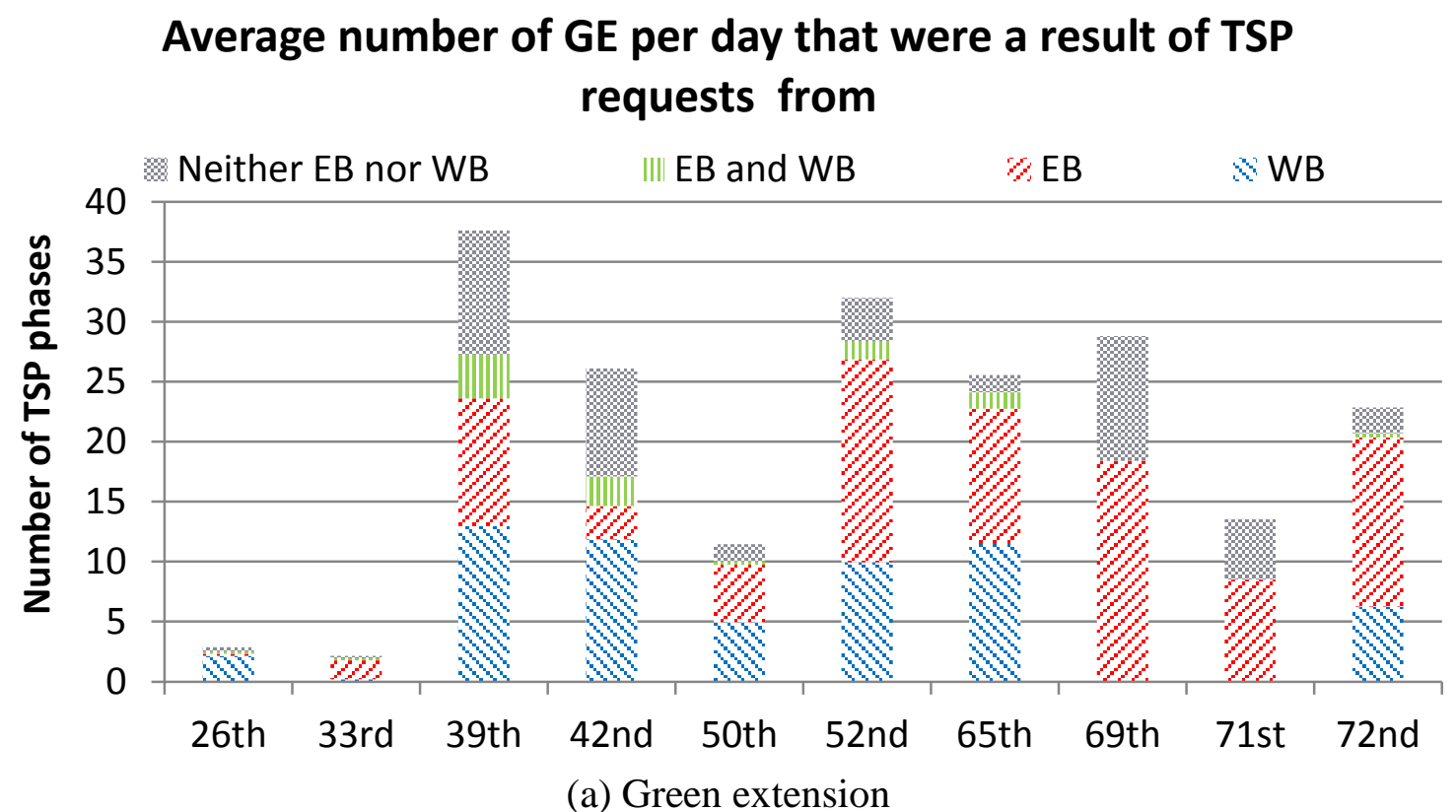




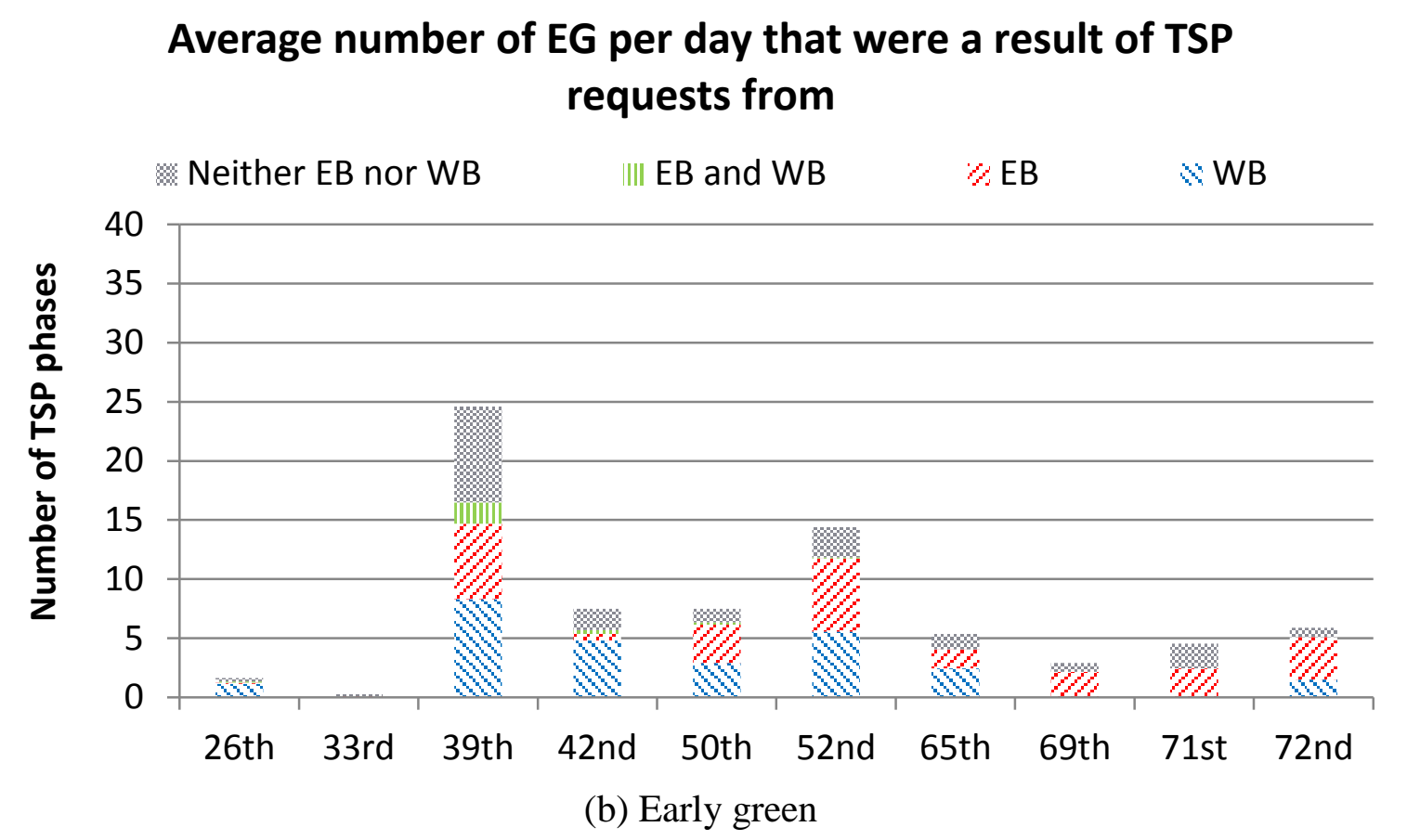

Figure 4-9 Average number of GE and EG phases per day

\subsection{TSP Effectiveness}

The previous section investigated the relationships between TSP requests and TSP phases. However, even if a bus TSP request resulted in the granting of a TSP phase in the same cycle, the bus may or may not benefit from this TSP phase; this TSP phase may be granted early, on-time or late. It is important to know the effectiveness of those TSP phases that resulted from TSP requests, and how many of them were granted early, ontime and late. This will be helpful for identifying more potential problems or improvement opportunities. TSP effectiveness can be defined separately for TSP requests and TSP phases. TSP phase effectiveness can be defined as the percent of TSP phases that benefited at least a TSP request; and TSP request effectiveness can be defined as the 
percent of TSP requests that benefit from at least a TSP phase. A bus TSP request is defined to benefit from a TSP phase if the TSP phase is granted on-time.

Similar to Figure 4-5, Figure 4-10 shows the relationships between TSP requests and four outcomes of TSP phases. This section tries to answer the following two questions:

1) For each bus TSP request, what are the probabilities that a TSP phase was granted early, on-time or late; or that no TSP phase was granted in a cycle?

2) For each TSP phase, what are the probabilities that this TSP phase was granted early, on-time or late as a result of one or more TSP requests; or that no TSP request existed within a cycle?

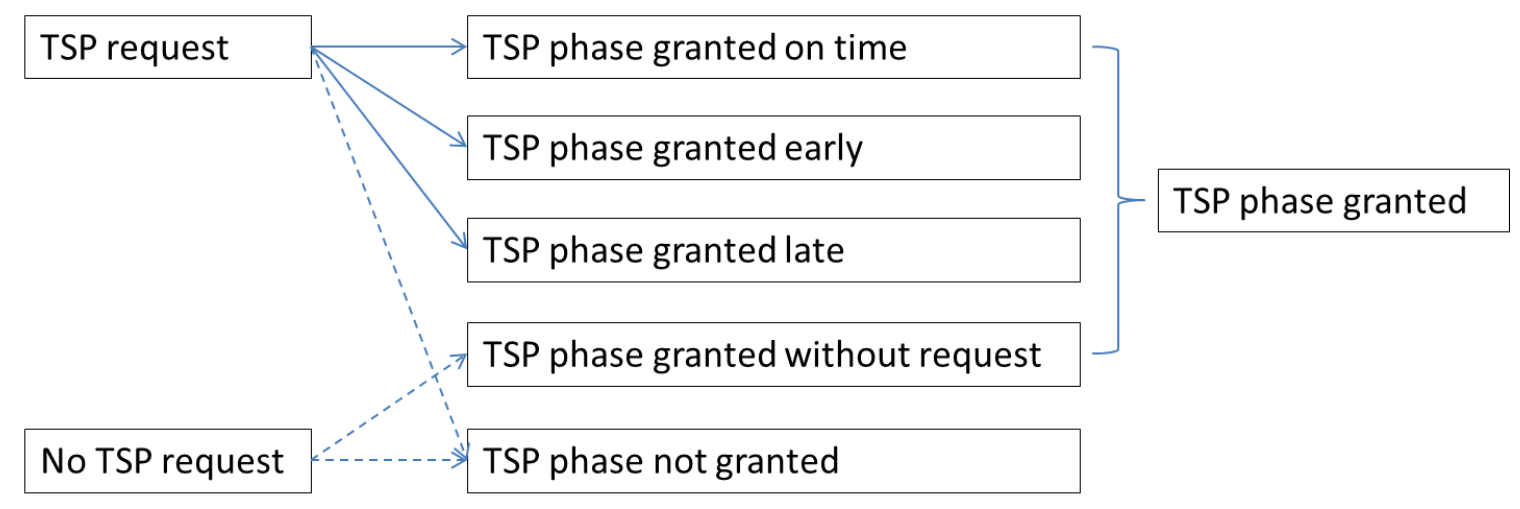

Figure 4-10 Detailed relationships between TSP requests and TSP phases

Figure 4-11 and Figure 4-12 explain when a TSP phase is granted early, late, on time or without any TSP request in the same cycle. For example, the GE (or EG) phase in cycle 
(2) in Figure 4-11 (or Figure 4-12) is late for bus "a"; it is on-time for bus "b"; and it is early for bus "c". All other cycles have been explained in Figure 4-6 and Figure 4-7.

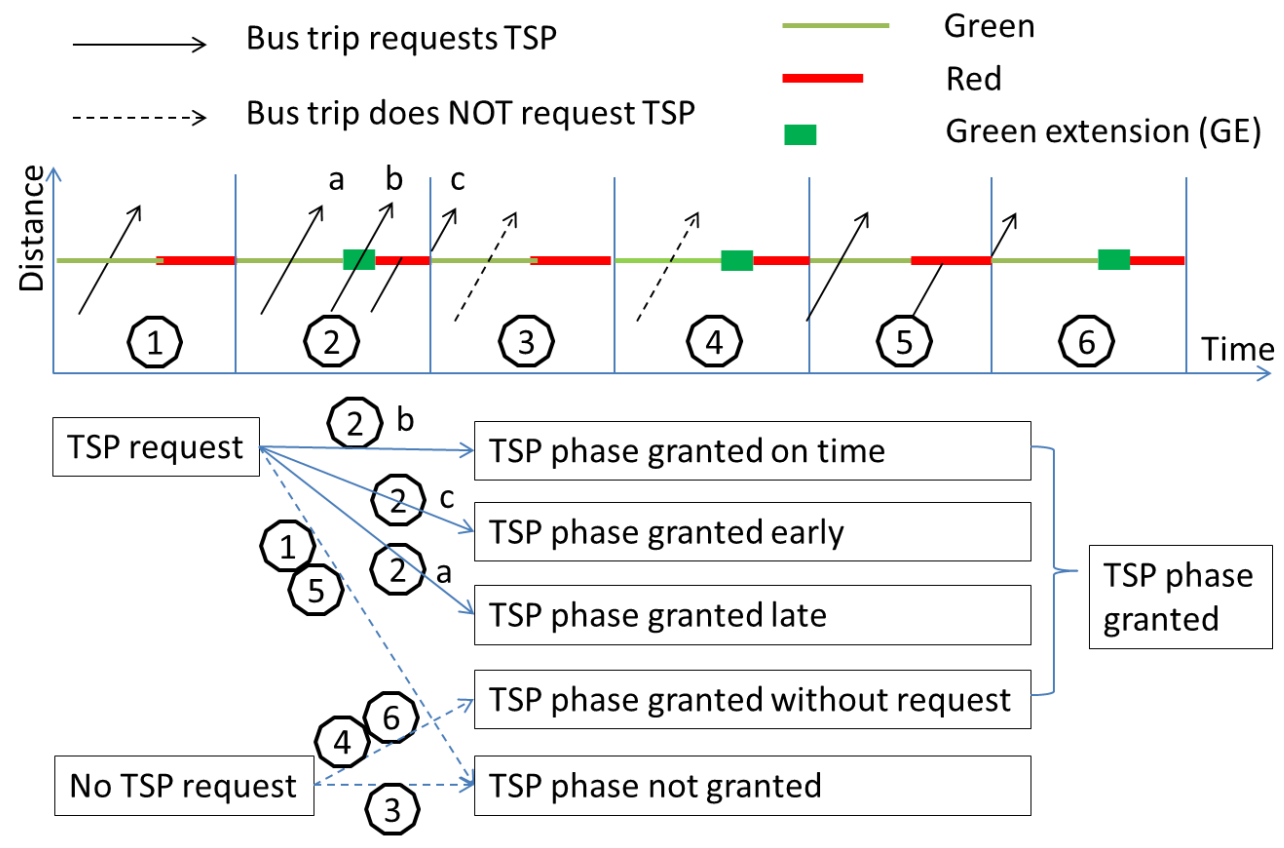

Figure 4-11 Detailed relationships between TSP requests and green extension phases

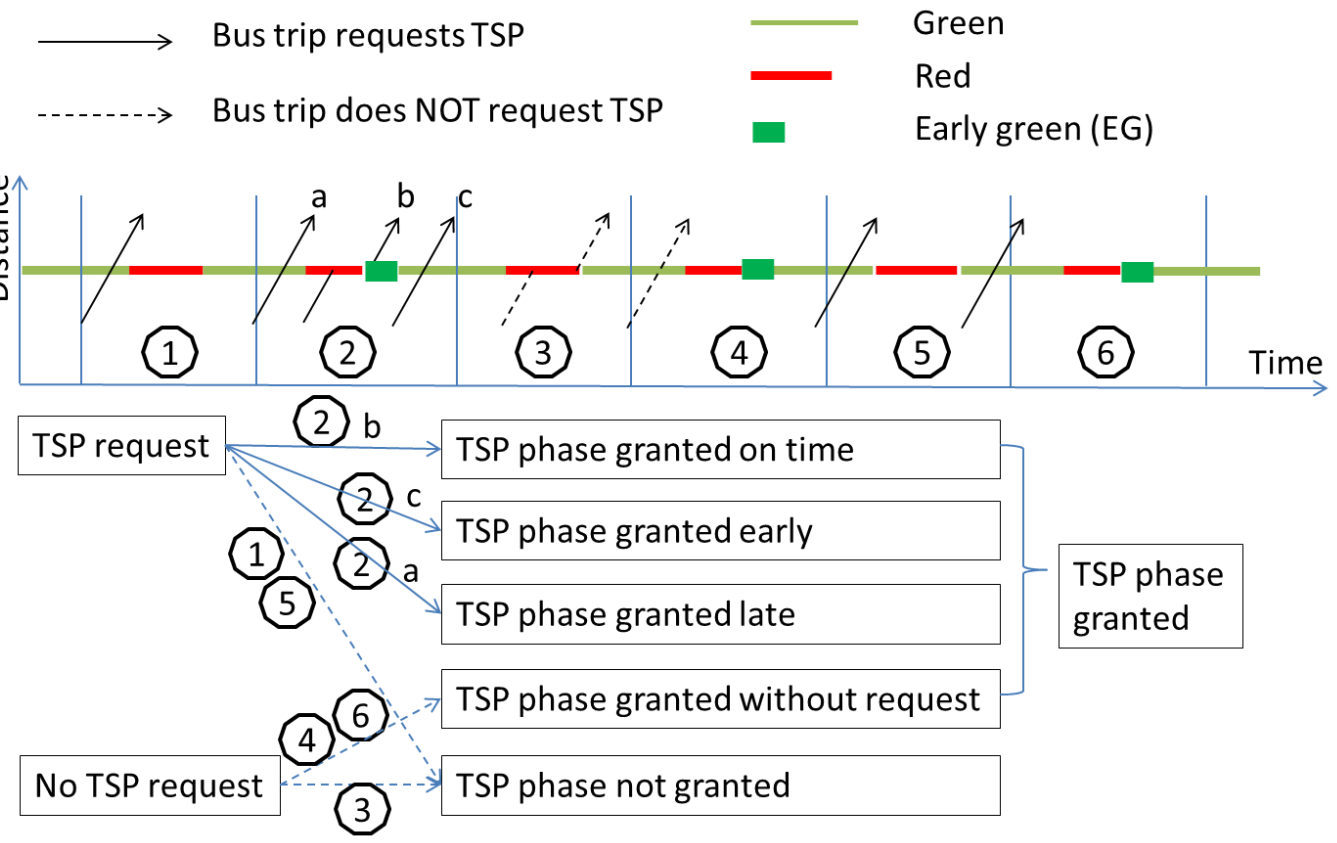

Figure 4-12 Detailed relationships between TSP requests and early green phases 


\subsubsection{TSP request effectiveness}

Because a bus that requests TSP can benefit from a GE phase only when the bus arrives at an intersection during the GE phase, the expected probability of a bus benefiting from the GE phase can be estimated by the ratio between the GE phase duration and the cycle length, assuming bus arrival pattern at the intersection is uniform. Similarly, a bus that requests TSP can benefit from an EG phase if the bus arrives at an intersection during the regular red phase, the expected probability of a bus benefiting from an EG phase can be estimated by the ratio between the regular red phase duration and the cycle length, assuming bus arrival time at the intersection is uniform and traffic conditions are unsaturated. In saturated traffic conditions, buses that arrive at the tail of a queue after the end of the EG phase may also benefit from the EG phase. Figure 4-13 shows the expected probabilities that a bus can benefit from a GE phase and from an EG phase, based on the observed median GE phase duration, regular red phase duration and cycle length. On average, a bus that requests TSP has $6 \%$ probability of benefiting from a GE phase and it has $5-50 \%$ probability of benefiting from an EG phase. 


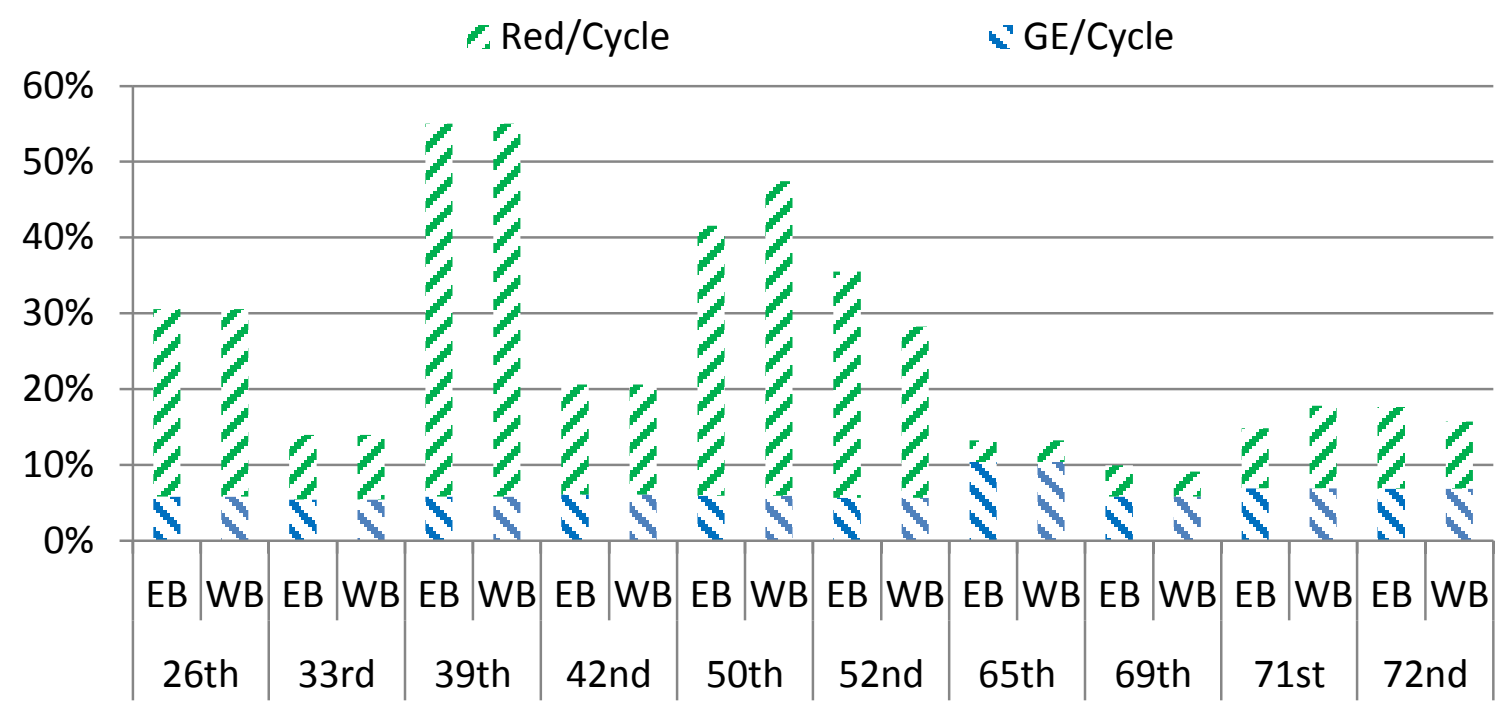

Figure 4-13 Probability that a bus TSP request can benefit from a TSP phase

Figure 4-14 shows the actual probabilities that a TSP request resulted in the granting of an early, on-time, and late GE phase, and that no GE phase was granted in the same cycle. Note that the probability that a TSP request does not result in a GE phase includes the probability that the TSP request results in an EG phase. Results indicate a low performance of GE phases because too many unnecessary (late) GE phases were granted, and the actual probability of a TSP request benefiting from a GE phase is much lower $(1.5 \%)$ than the expected probability $(6 \%)$. This may indicate a problem with the TSP control logic. For example, a GE phase will be granted irrespective of whether a TSP request is received in the beginning of a regular green phase or at the end of a regular green phase. It may also indicate a problem with the TSP request deactivation. For example, a TSP call in the signal controller may not have been canceled even if a bus has already passed the intersection. 


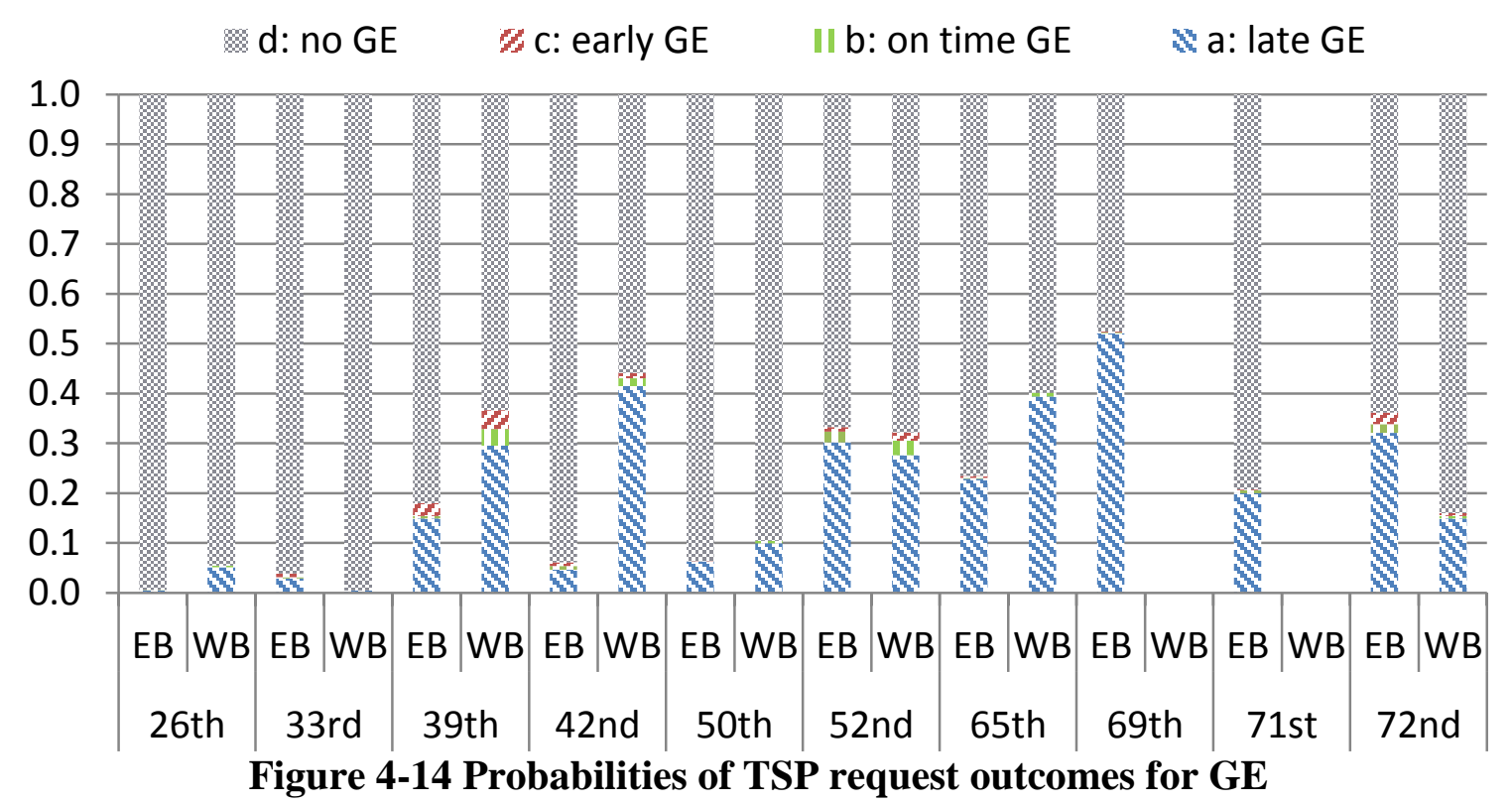

Figure 4-15 shows the actual probabilities that a TSP request resulted in the granting of an early, on-time, and late EG phase, and that no EG phase was granted. Note that the probability that a TSP request does not result in an EG phase includes the probability that the TSP request results in a GE phase. Results show that, on average, a bus TSP request has a $5-15 \%$ probability of benefiting from an EG phase and $0-15 \%$ probability of resulting in an EG phase to be granted early. EG phases that were granted early might also be beneficial to buses because queuing delay can be reduced due to EG phases. Another interesting finding is that EG phases are more effective at minor intersections than at major intersections. For example, at $42^{\text {nd }}$ Ave. WB direction, $69^{\text {th }}$ and $71^{\text {st }}$ Ave. EB direction, and $65^{\text {th }}$ and $72^{\text {nd }}$ Ave. both directions, the actual probabilities that a TSP request benefited from an $\mathrm{EG}$ phase are close to the expected probabilities as shown in Figure 4-13. However, at $26^{\text {th }}, 39^{\text {th }}, 50^{\text {th }}$ and $52^{\text {nd }}$ Ave. both directions, the measured 
probabilities that a TSP request benefited from an EG phases are much less than the expected probabilities.

In general, A TSP request has very high probability of resulting in an unnecessary (late) GE phase but very low probability of benefiting from a GE phase. In contrast, a TSP phase has high probability of benefiting from an EG phase and low probability of resulting in an unnecessary EG phase.

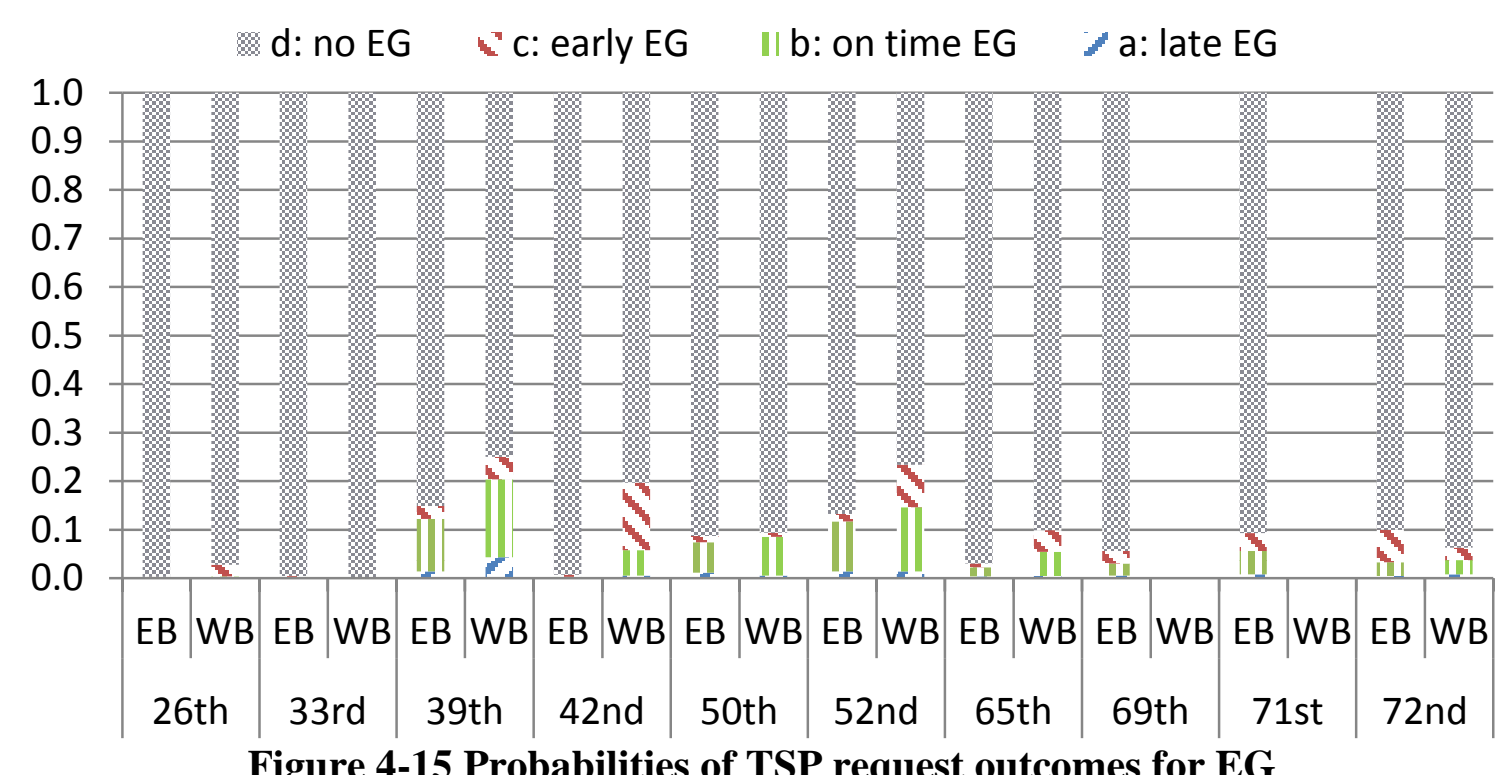

\subsubsection{TSP phase effectiveness}

The above section presents evaluation results of the TSP request effectiveness and provides some information about the probabilities of early, on-time and late TSP phases. However, it is also worthwhile evaluating the TSP effectiveness from the perspective of 
TSP phases as opposed to TSP requests. The probabilities that a TSP phase was granted early, on-time, late or in a different cycle are calculated to measure the TSP phase effectiveness.

GE phase effectiveness and EG phase effectiveness are shown in Figure 4-16 and Figure 4-17. The rest of each bar is the percent of TSP phases without any TSP request within the same cycle. Results vary significantly across intersections and by direction. Figure 4-16 shows that, on average, a GE phase has $64 \%$ probability of being late, $28 \%$ probability of being in a different cycle, $5 \%$ probability of being on-time and $3 \%$ probability of being early. This means that $95 \%$ of the GE phases are not effective and most of them are late. Results clearly indicate a problem with the GE phases. This might be a TSP control logic problem or a TSP request detection/deactivation problem. Figure 4-17 shows that, on average, an EG phase has $40 \%$ probability of being on-time, $30 \%$ probability of being early, $28 \%$ probability of being in a different cycle and $2 \%$ probability of being late. Therefore, EG phases are much more effective than GE phases. 

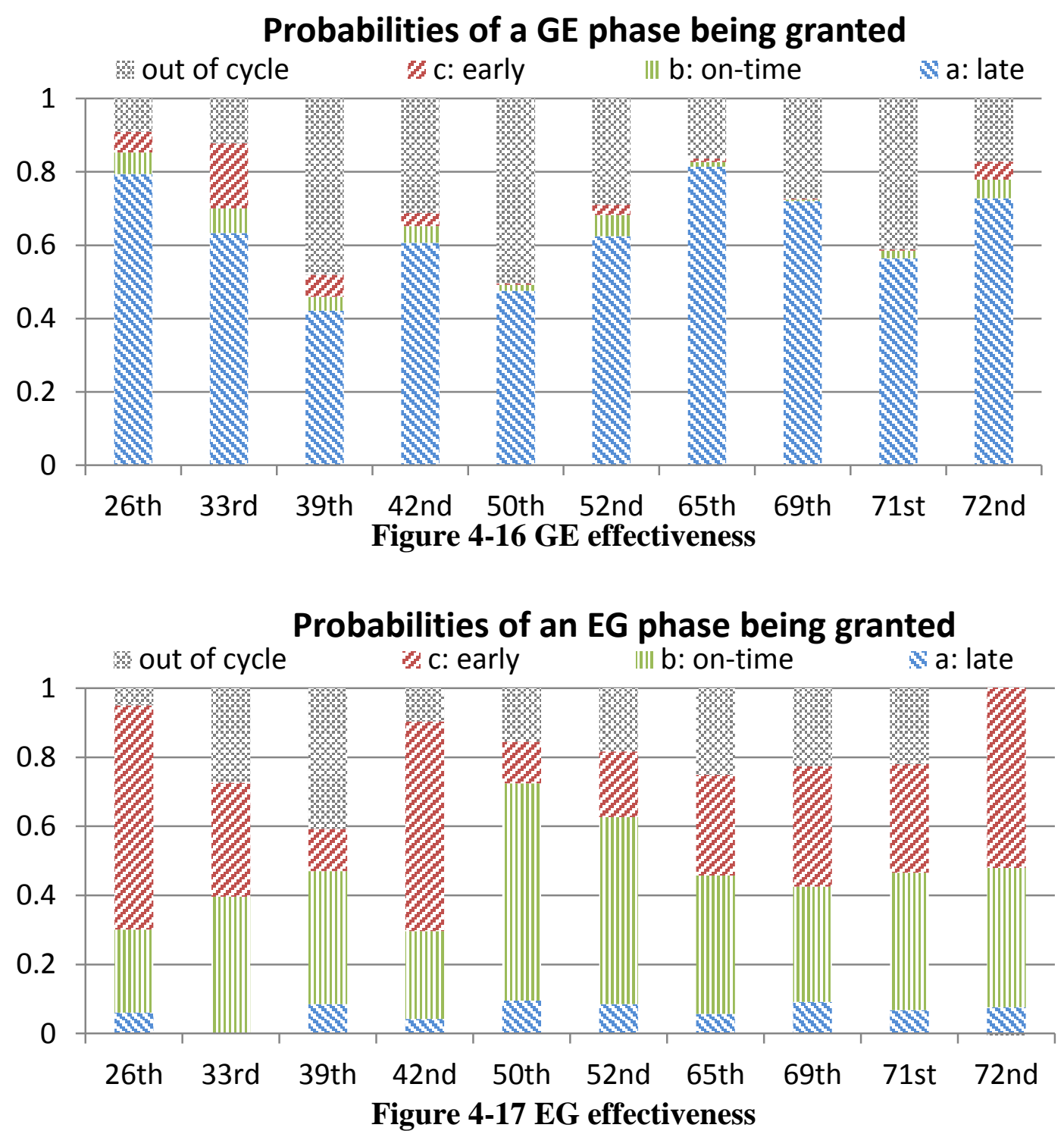

\subsection{TSP Benefits}

The above section shows that a TSP request is less likely to benefit from a GE phase than from an EG phase even though there are more GE phases. However, if a bus benefits from a GE phase, the time savings will be the time interval between the arrival time of this bus at the intersection and the end time of the following red phase. If a bus benefits 
from an EG phase, the maximum time savings will be the EG phase duration. Because red phase duration is longer than EG phase duration in most of the intersections, the time savings for a bus that benefits from a GE phase is usually higher than when it benefits from an EG phase. Therefore, it is important to measure the benefit (time savings) for each TSP request. On the other hand, because TSP phases can cause additional delay to side street traffic, it is also important to measure the benefit (time savings) of each TSP phase, and to compare whether the benefits for the bus and its onboard passengers outperform the cost (delays) to side street vehicles. Time savings for the bus and the passengers are estimated based on the algorithm in Chapter 2. The estimation of time savings for other vehicles on the major street and delays to vehicles on the minor street will be shown in the following sections.

\subsubsection{Estimated benefit per TSP request}

Figure 4-18 shows that the estimated time savings per TSP request from a GE phase is 0.3 seconds, and the estimated time savings per TSP request from an EG phase is 0.5 seconds. These estimated time savings are very low for both GE and EG phases because the probabilities of a TSP request benefiting from a TSP phase are very small at most of the intersections. Figure 4-19 shows that the estimated passenger time savings for a TSP request from a GE phase and from an EG phase are 7.5 and 10 seconds, respectively. Results indicate that a TSP request has slightly higher time savings from an EG phase than from a GE phase. 


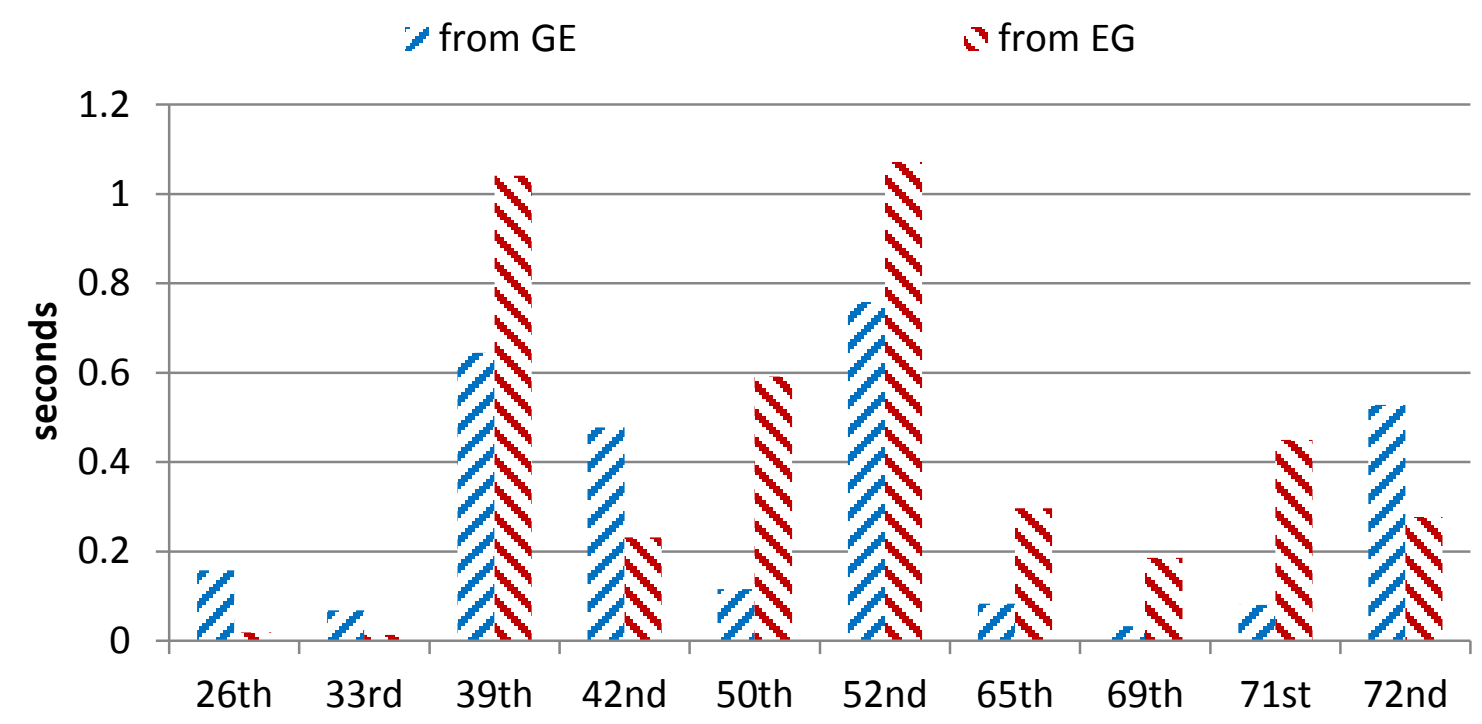

Figure 4-18 Estimated bus time savings per TSP request

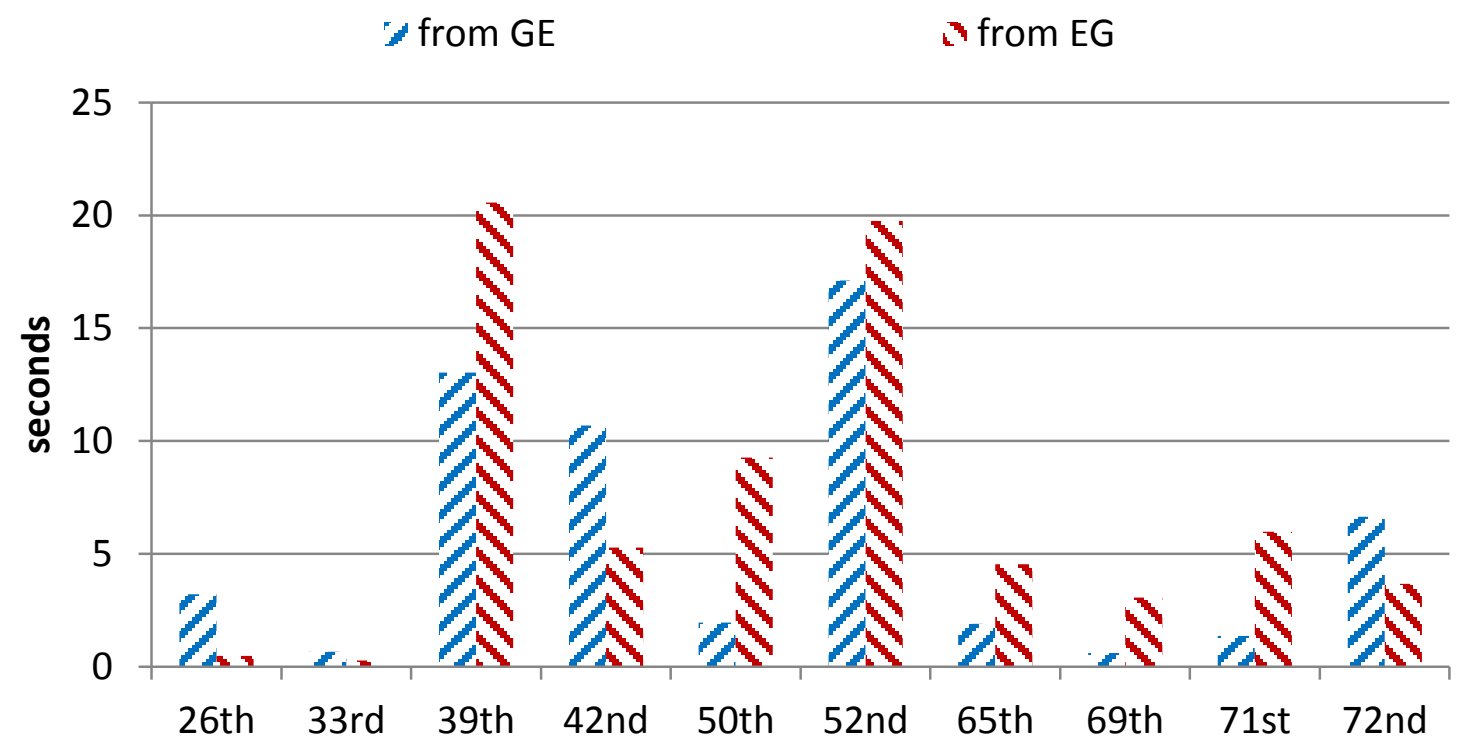

Figure 4-19 Estimated total passenger time savings per TSP request

\subsubsection{Estimated benefit per TSP phase}


Although the expected benefit from a GE phase is higher than from an EG phase, Figure 4-20 (a) and (b) show that the estimated bus time savings per GE phase and per EG phase are 1 and 4 seconds, respectively. Similarly, Figure 4-21 (a) and (b) show that the estimated passenger time savings for each granted GE phase and EG phase are 20 and 90 seconds, respectively. This may be because too many non-beneficial GE phases were granted than EG phases.

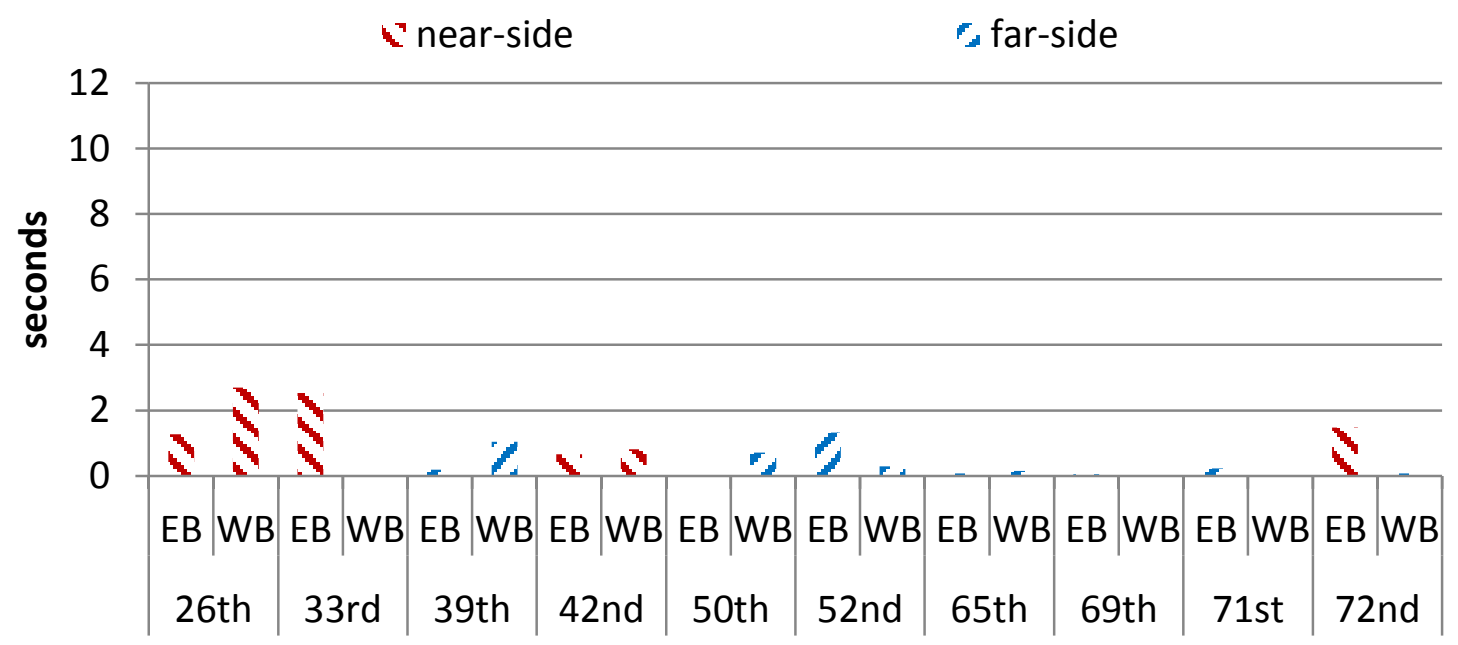

(a) GE

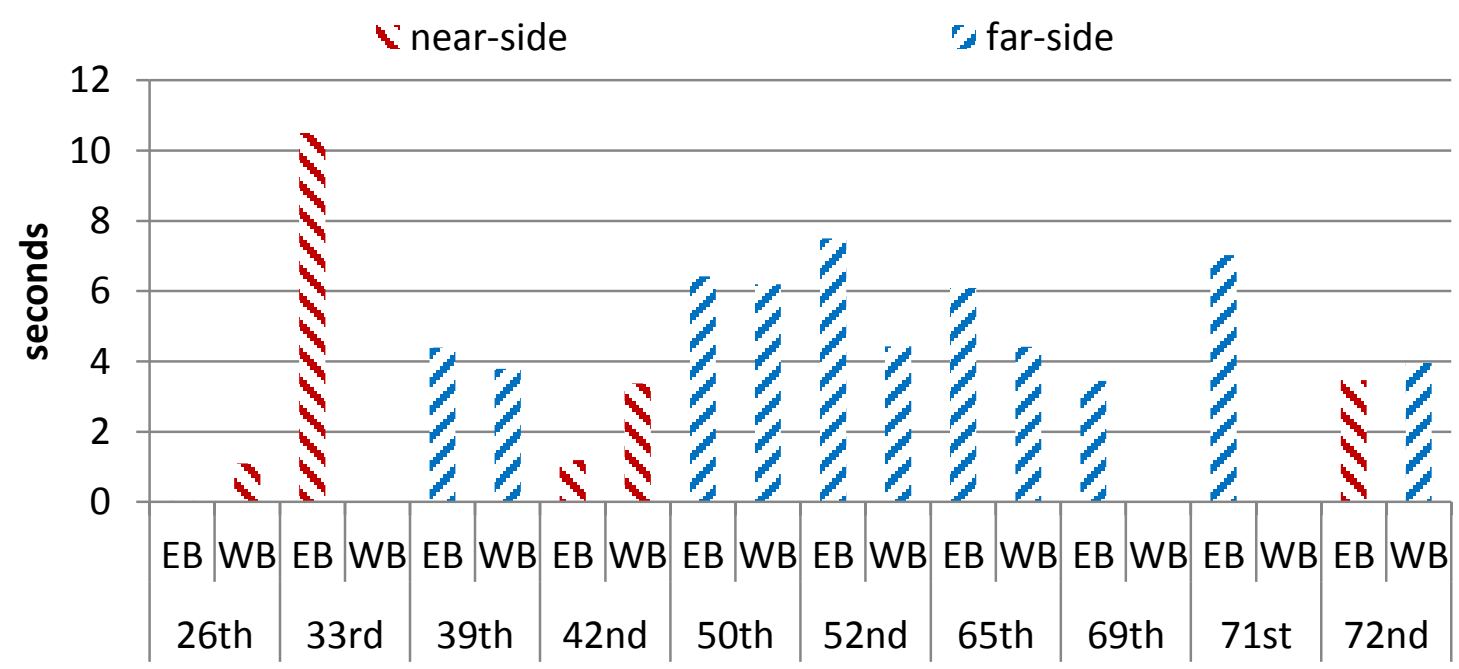

(b) EG

Figure 4-20 Estimated bus time savings per TSP phase 


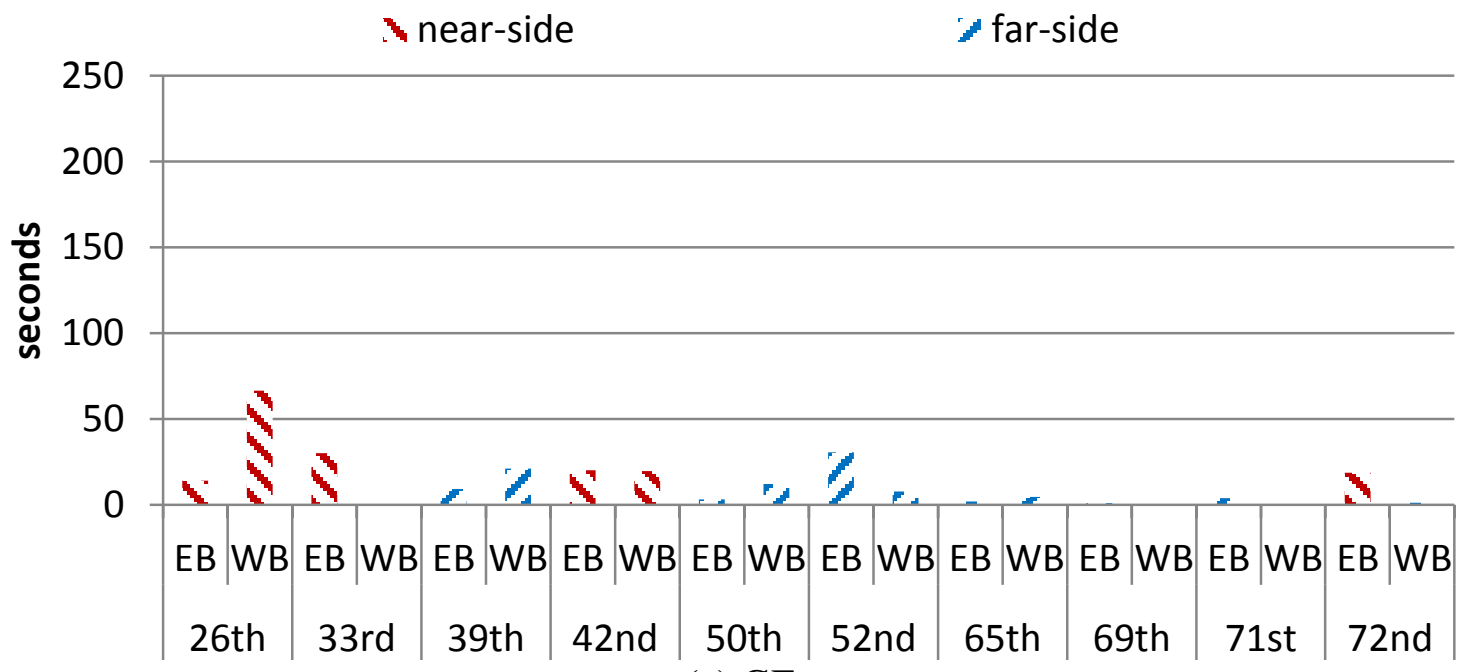

(a) GE

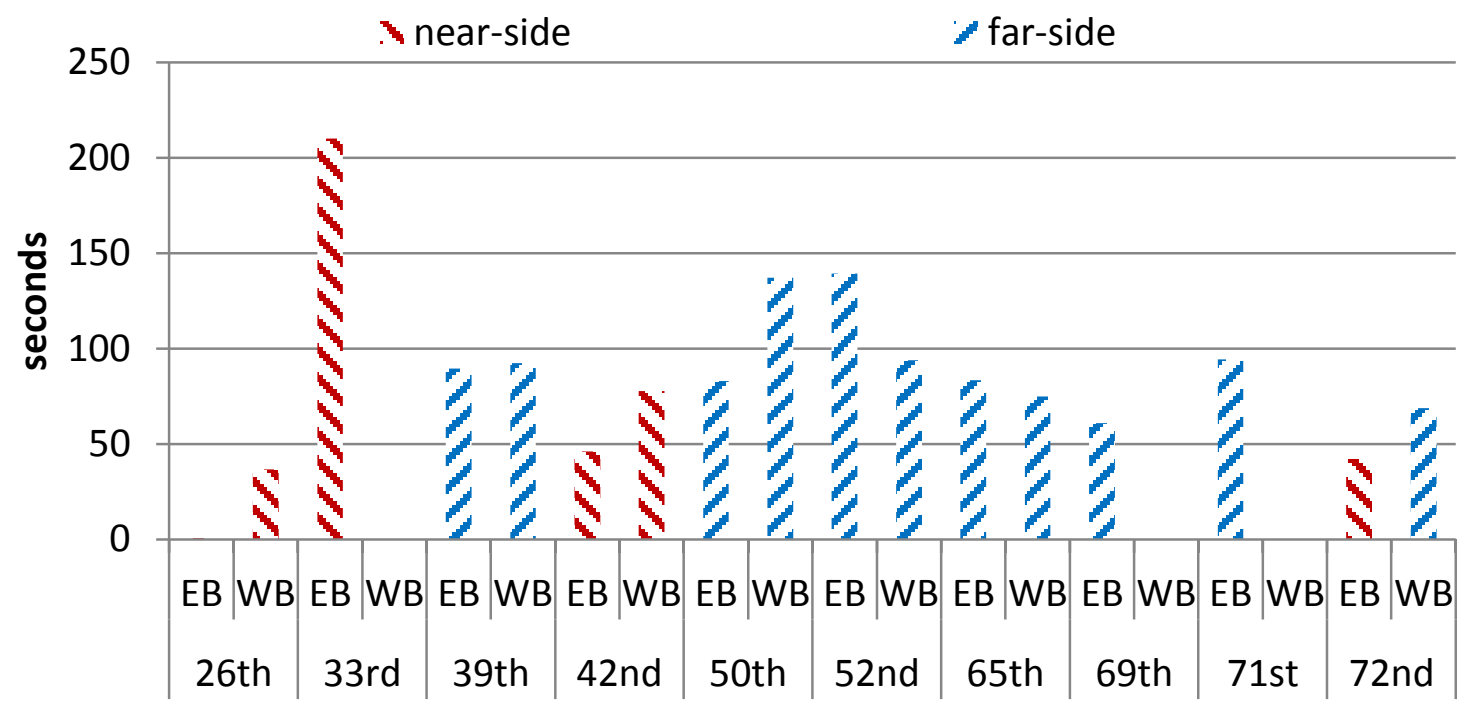

(b) EG

Figure 4-21 Estimated total passenger time savings per TSP phase

According to Smith et al. (2005), TSP works better at far-side stops because bus arrival time prediction is more reliable at far-side stops. However, our results did not show clear differences in the estimated bus time savings and passenger time savings per TSP phase between near-side stops and far-side stops. This finding does not indicate that near-side 
and far-side stop configurations have no impact on TSP performance, because there are only six near-side stop segments and four of them may have TSP setting problems.

\subsubsection{Time savings and delay between buses and other vehicles}

It is also necessary to compare time savings for the buses and vehicles on Powell Blvd. due to a TSP phase with delays for vehicles on the side streets due to the TSP phase. Assuming vehicle and bus arrival rates at intersections are uniform (vehicle platooning arrival pattern was not considered in this case), traffic conditions are unsaturated in all four approaches, and regular green phase and red phase durations will not change if a GE phase or an EG phase is granted, the total time savings (TTS) for vehicles on the major street and the total delay (TD) for vehicles on the side streets can be estimated by the following equations:

$$
\begin{aligned}
& T T S=\frac{q_{1} \cdot q_{2}}{2\left(q_{2}-q_{1}\right)}\left(2 \cdot \operatorname{Red} \cdot T S P-T S P^{2}\right) \\
& T D=\frac{q_{1} \cdot q_{2}}{2\left(q_{2}-q_{1}\right)}\left(2 \cdot \operatorname{Red} \cdot T S P+T S P^{2}\right)
\end{aligned}
$$

The derivations of these equations are illustrated in Figure 4-22. $q_{2}$ is the discharge flow, it is assumed to be 1800 vehicles per hour per lane. $q_{1}$ is the vehicle arrival flow from an approach of an intersection. Red is the regular red phase duration for an approach of an

intersection. TSP is the median TSP phase duration (either GE or EG) for an intersection. 

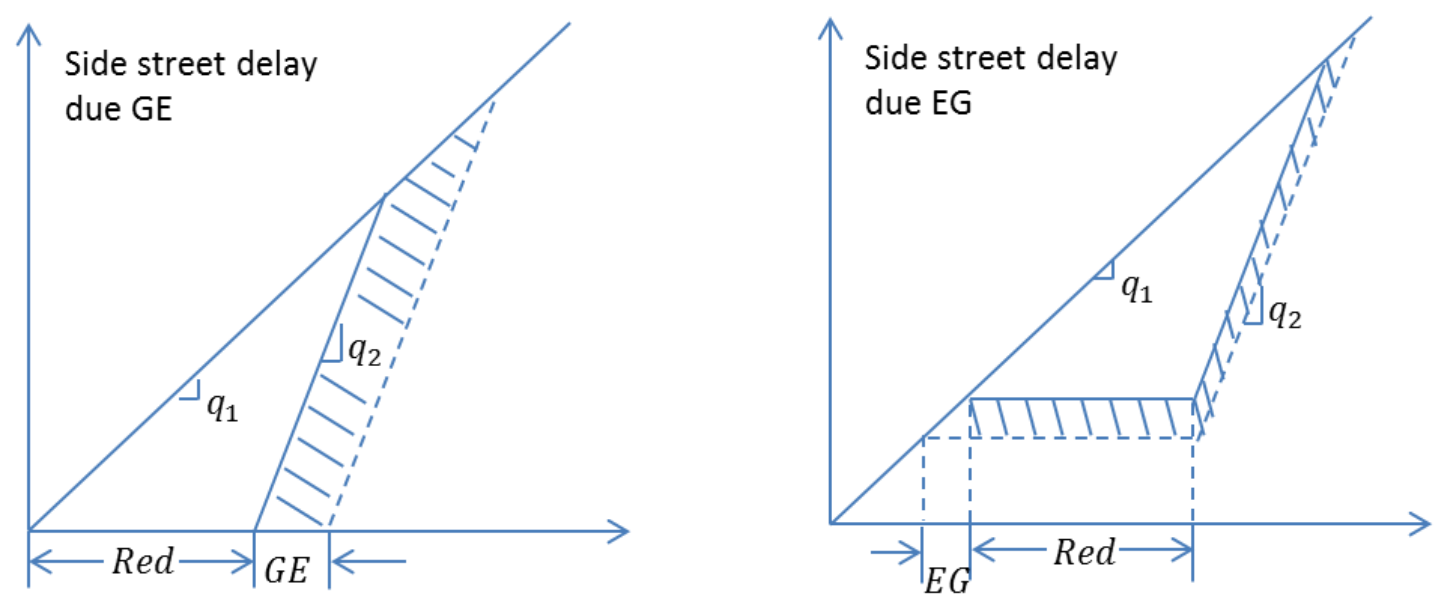

$$
T D=\frac{q_{1} \cdot q_{2}}{2\left(q_{2}-q_{1}\right)}\left(2 \cdot \operatorname{Red} \cdot G E+G E^{2}\right)
$$

$$
T D=\frac{q_{1} \cdot q_{2}}{2\left(q_{2}-q_{1}\right)}\left(2 \cdot \operatorname{Red} \cdot E G+E G^{2}\right)
$$
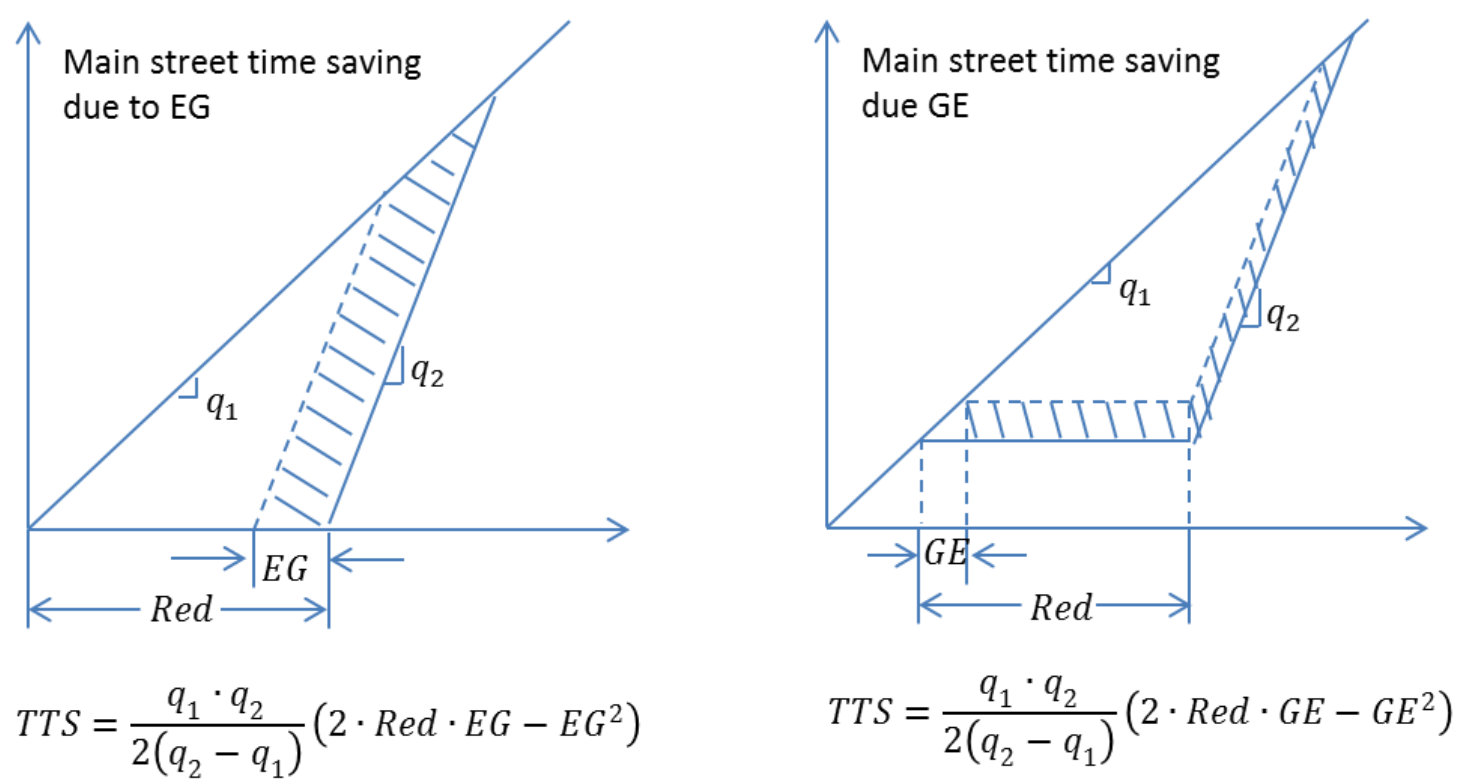

$T T S=\frac{q_{1} \cdot q_{2}}{2\left(q_{2}-q_{1}\right)}\left(2 \cdot \operatorname{Red} \cdot E G-E G^{2}\right)$

Figure 4-22 Illustration of major street time savings and side street delay 
EB/WB time savings vs. NB/SB delay per granted EG

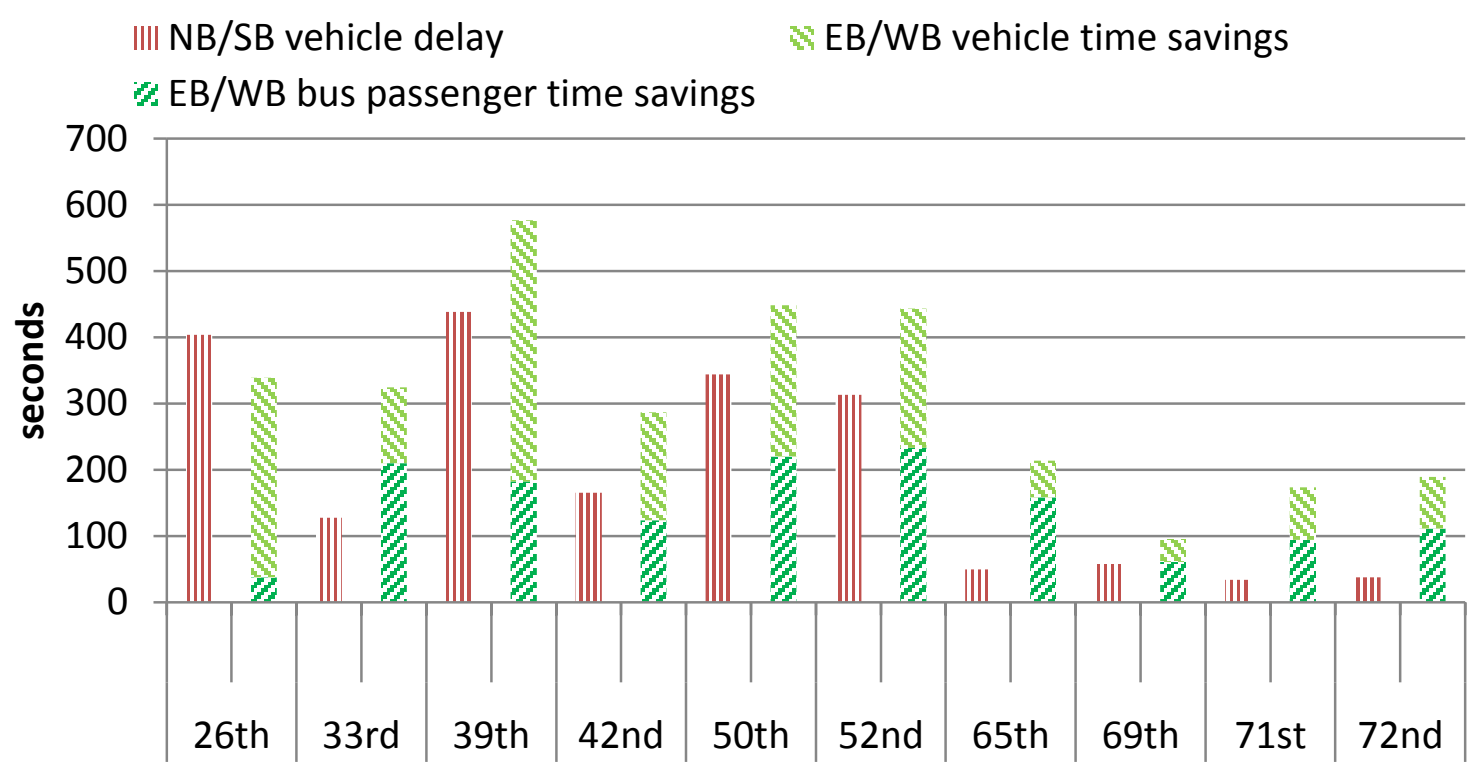

(a) EG

\section{EB/WB time savings vs. NB/SB delay per granted GE}

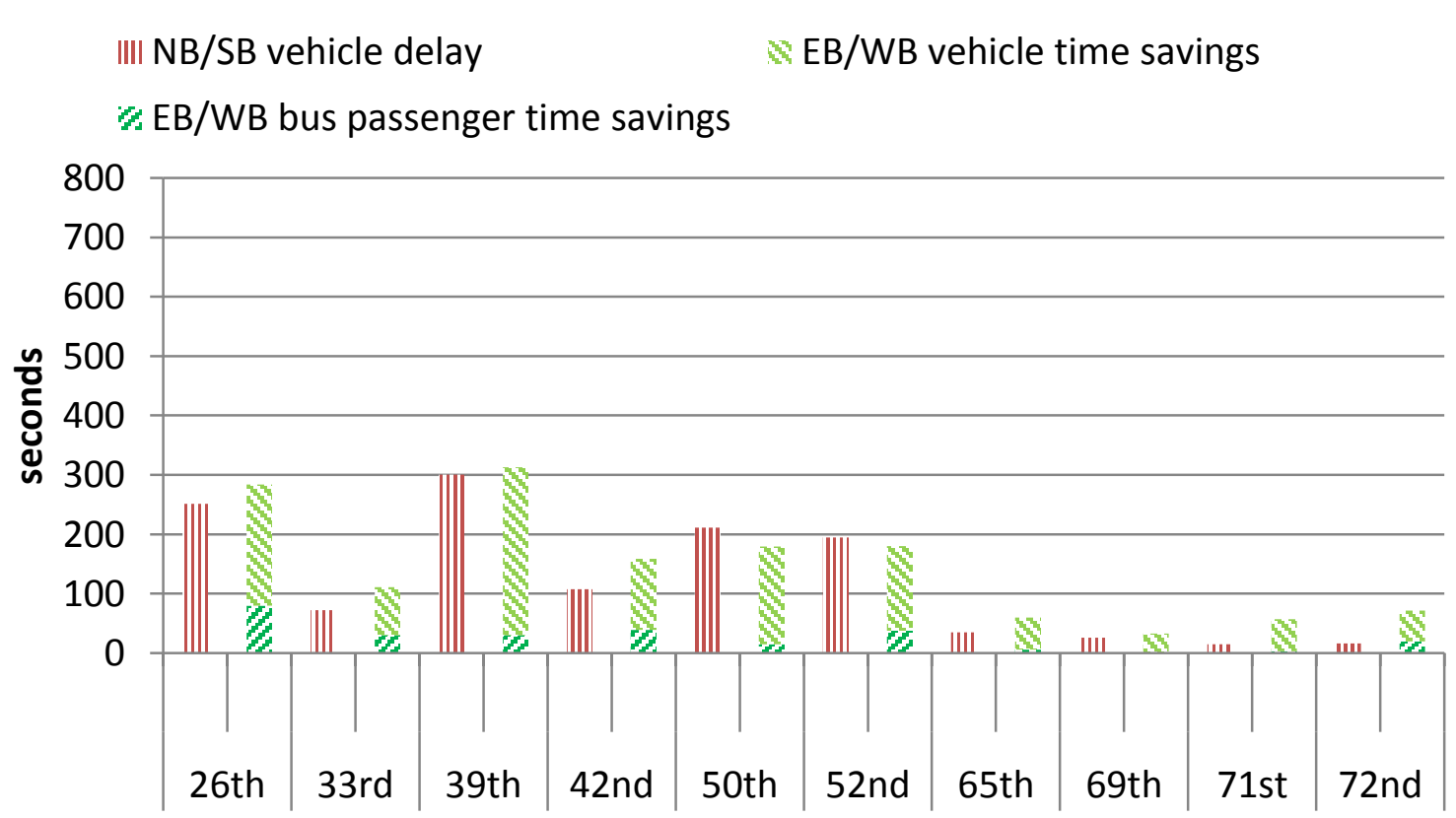

(b) GE

Figure 4-23 Total passenger time savings and delay per TSP phase 
Assuming all other vehicles are single occupancy vehicles, the total estimated vehicle delays for the NB and SB approaches, the total vehicle time savings for the EB and WB approaches, and the passenger time savings are shown in Figure 4-23. Results show that an EG phase creates more side street delay than a GE phase because the EG phase duration is longer than the GE phase duration. For each EG phase, the bus passenger time savings is slightly less than the total vehicle delay on the side street for intersections west of $52^{\text {nd }}$ Ave., but the sum of the bus passenger time savings and the total vehicle time savings on the major street is much higher than the side street vehicle delay at all intersections. For each GE phase, the sum of the bus passenger time savings and the vehicle time savings on the major street is almost equal to the vehicle delay on the side street.

Because the average duration of a GE phase is smaller than the average duration of an EG phase, it is worth to compare the efficiencies between them (total time savings and delays per second GE phase and EG phase). According to equations shown in Figure 4-22, the total vehicle delays on the minor street increase concavely $\left(G E^{\prime}>0, G E^{\prime \prime}>0, E G^{\prime}>\right.$ 0 and $E G^{\prime \prime}>0$ ), which means the total vehicle delay incremental rate increases when TSP phase duration increases. However, the total time savings for vehicles on the major street increase convexly $\left(G E^{\prime}>0, G E^{\prime \prime}<0, E G^{\prime}>0\right.$ and $\left.E G^{\prime \prime}<0\right)$ when $(0<$ GE or $E G<2$ Red), which means the total vehicle time savings incremental rate decreases when TSP phase duration increases. Bus passenger time savings will also increase if GE or EG duration increases; however, the incremental rate is unknown because bus passenger time savings are estimated by comparing each pair of bus 
departure time and arrival time with TSP phase start and end times. Therefore, assuming total bus passenger time savings and other vehicles time savings on the major street and total delays for vehicles on the minor street all increase linearly with GE and EG durations, the average total time savings and total delays per second GE phase and per second EG phase can be estimated by dividing the total time savings and delays by TSP durations ( 7 seconds for GE and 11 seconds for EG). The results are shown in Figure 4-24.

Results show that the total time savings and delays for non-bus vehicles per second GE phase and per second EG phase are very similar (less than 2 seconds difference), which means the nonlinear effect of TSP phase duration on non-bus vehicles time savings and delays is very small. However, the total bus passenger time savings per second EG phase is much higher than the total bus passenger time savings per second GE phase. Therefore, after controlling for TSP phase duration, EG phases are more efficient than GE phases. 
EB/WB time saving vs. NB/SB delay per second EG phase

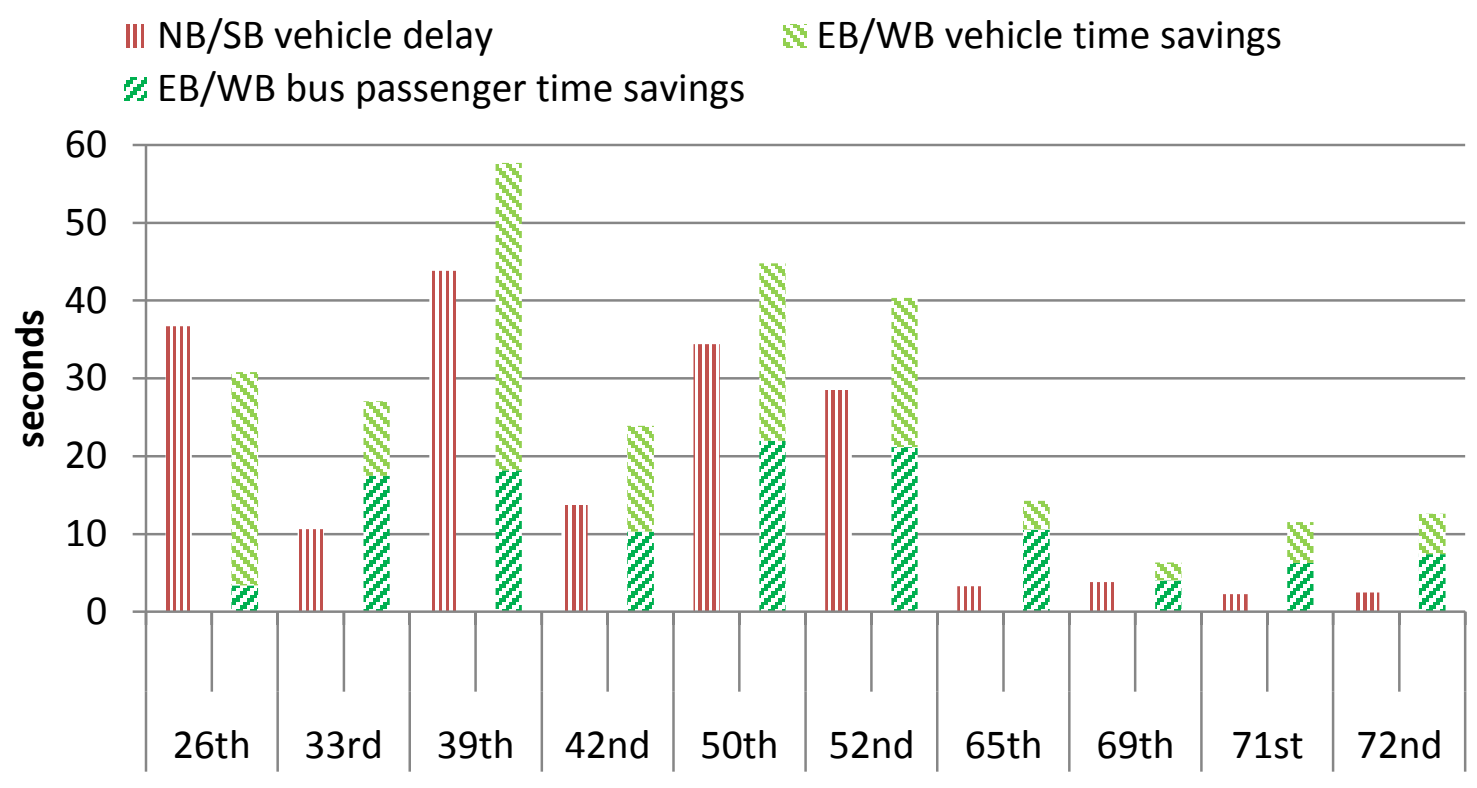

(a) Green extension

\section{EB/WB time savings vs. NB/SB delay per second GE phase}

IIII NB/SB vehicle delay

$\mathrm{NEB} / \mathrm{WB}$ vehicle time savings

Z EB/WB bus passenger time savings

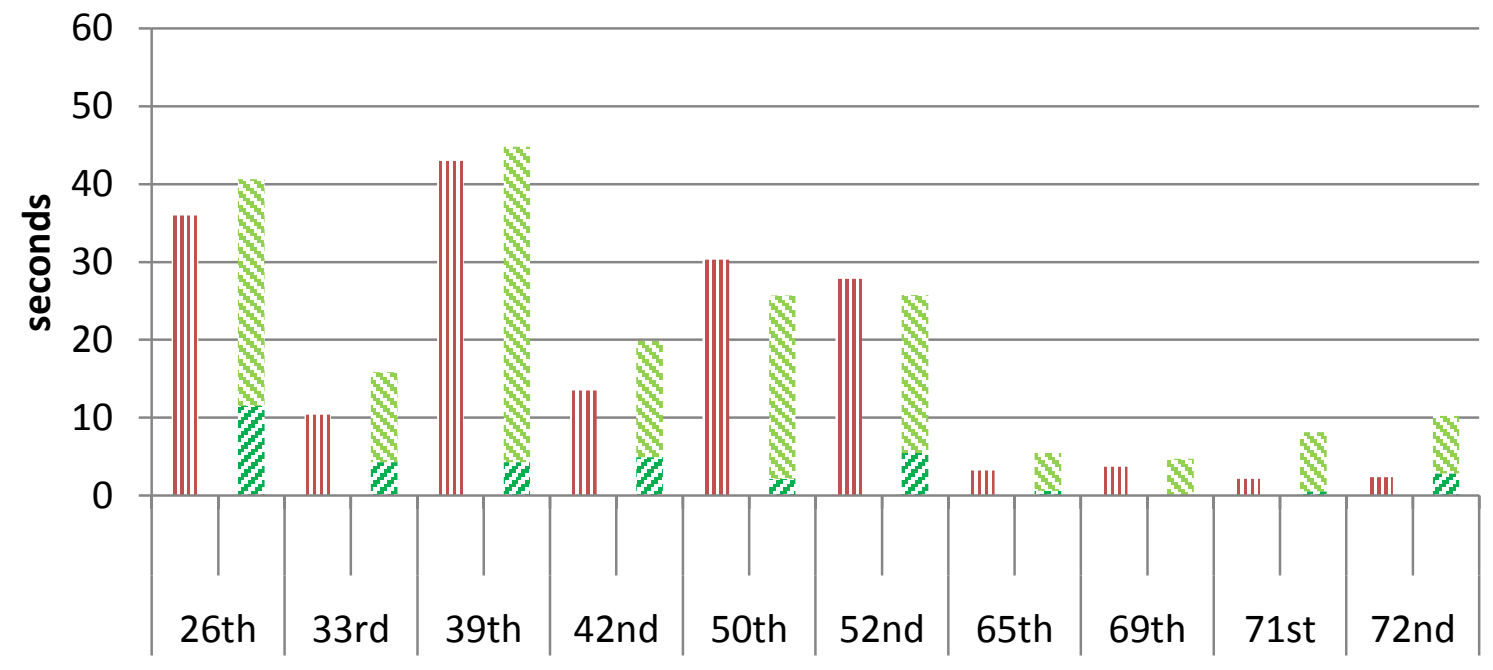

(b) Early green

Figure 4-24 Total passenger time savings and vehicle delays per second TSP phase 


\subsection{Summary}

In summary, TSP performance is evaluated at the intersections between $26^{\text {th }}$ Ave. and $72^{\text {nd }}$ Ave. on Powell Blvd. based on bus AVL/APC data and SCATS phase log data. This performance evaluation consists of three sections: 1) the relationships between TSP phases and TSP requests, which measure how timely TSP phases react to TSP requests; 2) TSP request and TSP phase effectiveness, which evaluate the percentages of TSP phases that are beneficial to TSP requests; and 3) TSP request and TSP phase benefits, which compare the estimated time savings for buses and passengers between GE and EG phases and compare the estimated time savings for bus passengers and other vehicles on the major street and the side street. Results indicate that EG phases have better performance than GE phases (higher percent of effective EG phases and higher estimated time savings) because many GE phases are granted late. Results also show that the time savings for bus passengers is less than the delay to side street vehicles due to a TSP phase; however, the total time savings of bus passengers and vehicles on the major street is higher (or equal to) than the delay to side street vehicles for an EG phase (or a GE phase). Additional discussion is included in Chapter 5 regarding the findings presented in this chapter. 


\section{CONCLUSIONS}

\subsection{Summary of Findings}

This study has analyzed the joint impact of intersection signal delay, traffic conditions and bus stop location on bus stop-to-stop travel time across an intersection and evaluated the TSP system performance on reducing bus delay at the stop-to-stop level. This study is conducted based on the integration and analyses of three databases including: bus AVL/APC data, SCATS signal phase log data and traffic count data. A bus stop-to-stop trip database was created by integrating these three databases and was utilized to study factors that affect bus travel time variability and evaluate the TSP system performance.

\subsubsection{Bus stop-to-stop travel time modeling}

To study the simultaneous impacts of intersection signal delay, traffic conditions and bus stop location on bus stop-to-stop travel time across an intersection, several bus stop-tostop travel time regression models were estimated. First, a bus departure-to-arrival time model was estimated for far-side segments to study the impacts of intersection signal delay and traffic conditions on bus travel time by eliminating passenger activity effects. Then, a bus arrival-to-arrival time model was estimated for far-side segments to validate the robustness of estimated variable coefficients. Another bus arrival-to-arrival time model was estimated for all stop-to-stop segments (both far-side and near-side segments) 
to investigate how the impacts of intersection signal delay and traffic conditions are affected by bus stop location type. Finally, a series of bus stop-to-stop travel time regression models were estimated on each individual segment to examine how these impacts are affected by intersection signal timing plans and geometric characteristics.

The three pooled bus stop-to-stop travel time models explained $74-78 \%$ of the travel time variation. On average, $50 \%$ of the bus stop-to-stop travel time variability is due to signal delay. Therefore, signal delay is the primary source of bus travel time variability across an intersection. Only one study in the literature, Albright and Figliozzi (2012a), has analyzed bus travel time at the stop-to-stop segment level. The estimated regression model explained $61 \%$ of the bus stop-to-stop travel time variability. The effect of signal delay was estimated as a constant value: each signalized intersection adds 10 seconds to bus stop-to-stop travel time. The effect of signal delay on bus travel time variability was not provided. Another study, Mazloumi et al., (2010), estimated the effect of signalized intersection density on route-level bus travel time variation. Results showed that each additional number of intersections per kilometer increases bus travel time variability by $22 \%$.

The average signal delay for a bus passing through an intersection was found to be $70 \%$ of the red phase duration for the bus travel direction at the intersection, and this ratio is higher at intersections with larger RC ratios (red phase duration divided by cycle length). This may be because intersections with large $\mathrm{RC}$ ratios tend to have long queues, and buses may experience additional queuing delay. Several studies have estimated the effect 
of signal delay to bus travel time (Abkowitz and Engelstein, 1984; McKnight et al., 2004; Albright and Figliozzi, 2012b; El-Geneidy et al., 2009; Figliozzi and Feng, 2012), they found that each additional number of intersections increases bus travel time by 8-26 seconds. However, these studies only estimated the average signal delay due to each additional intersection, and intersection signal timing characteristics were not considered. The signal delay variables used in our study incorporate intersection signal timing characteristics such as red phase duration and RC ratio.

The effect of traffic conditions on bus stop-to-stop travel time was found to be significant and positive. On average, for each 1,000 vehicles per hour increase in traffic volume (all lanes), bus stop-to-stop travel time increases by 2 seconds. The estimated coefficient is lower than expected. This may be because the 15-minute interval traffic volume measurement is not granular enough to represent the actual traffic conditions during the time a bus is traveling through an intersection. Also, the impact of traffic conditions was found to be significantly different by time of day and by stop-to-stop segment. In general, the impact of traffic conditions on bus travel time is higher at intersections with large RC ratios. Additional bus travel time delay was found during peak hours, but the travel time increment rate associated with traffic volume is smaller during peak hours. This is the first study that directly measures the impact of traffic volume on bus stop-to-stop travel time, although there is one study that analyzed the effect of traffic flow on bus travel time at the time point segment level (travel time between two time point bus stops). Mazloumi et al. (2012) found that traffic flow explained as much as 50\% of bus travel time variation and that the impact of traffic flow can be approximated by time of day variables with 
little loss in prediction accuracy. However, because passenger activity effects were not included in their model, it is very likely that the time of day variables accounted for both traffic and passenger activity effects. All other studies only used "time of day" and "direction of travel" as variables to indirectly measure the impact of traffic condition.

Travel distance has a significant and positive effect on bus travel time, as expected. The average travel speed is 26-29 mph without signal delay on this corridor. Previous studies also found significant effects of travel distance. However, the estimated coefficients vary substantially, the equivalent travel speeds range between $10 \mathrm{mph}$ and $32 \mathrm{mph}$ (Abkowitz and Engelstein, 1984; Bertini and El-Geneidy, 2004; El-Geneidy and Surprenant-Legault, 2010; El-Geneidy et al., 2011; Figliozzi and Feng, 2012; Strathman et al., 2002, 2000). This may be because bus route characteristics are different among these studies and different variables and model specifications were used.

The impacts of signal delay, traffic conditions and passenger activities on bus stop-tostop travel time differed significantly between near-side and far-side segments. This may be because near-side bus stops are very close to intersections, there is less of a queuing effect. Also, it is likely that some buses serve passengers at near-side stops when waiting for a red signal, the impacts of signal delay and passenger activities on bus travel time are less at near-side segments than at far-side segments. Texas Transportation Institute (1996), HCM (2010) and Kittelson \& Associates, Inc. (2013) discussed the advantages and disadvantages of near-side and far-side bus stop configurations. For example, far-side stops reduce conflicts between buses and right-turning vehicles, but buses may have to 
stop twice if they encounter a red signal; near-side stops may allow buses to serve passengers when waiting for a red signal, but buses are likely to get caught by a red signal after serving passengers, and vehicle queuing upstream of the intersection may create additional delay for buses to arrive at near-side stops. In general, it is suggested that far-side stops are preferable than near-side stops in terms of reducing bus delay at signalized intersections. Although this study and Albright and Figliozzi, (2012a) both found that bus stop-to-stop travel time is about 4 seconds less at near-side segments than at far-side segments, these results do not mean near-side bus stop configuration can help reduce bus travel time because bus delay due to intersection queuing effects was included in the bus stop-to-stop travel time for far-side segments but not included for near-side segments.

The impact of passenger activities on bus stop-to-stop travel time was found to be significant and positive. On average, each additional passenger boarding increases bus stop-to-stop travel time by 3.4 seconds for far-side segments and 1.9 seconds for nearside segments. Each additional lift use increases bus stop-to-stop travel time by 36.9 seconds for far-side segments and 24.8 seconds for near-side segments. The impact of passenger alighting activity was not significant. The smaller effects of passenger activities at near-side stops may be because some buses serve passengers when waiting for a red signal. Many studies have estimated the impacts of passenger activities, but results vary significantly. The estimated coefficient ranges for passenger boarding, alighting and lift use are: 0.6-6 seconds; 0.4-4 seconds; and 31-67 seconds (Abkowitz and Engelstein, 1984; Bertini and El-Geneidy, 2004; Figliozzi and Feng, 2012; McKnight 
et al., 2004; Slavin et al., 2013; Strathman et al., 2002, 2000). These large variations may be because studies have used different explanatory variables (e.g. the sum of boarding and alighting passengers and the square terms of boarding and alighting passengers) for passenger activities or different dependent variables (e.g. dwell time, travel time). It is also likely these studies have different route characteristics or fare payment systems. However, no study has estimated the coefficients of passenger activities for near-side and far-side stops separately.

\subsubsection{TSP performance evaluation}

Several performance measures were proposed in this study to evaluate how well the TSP system helps reduce bus delay through signalized intersections. These performance measures include: the percentage of TSP requests that lead to the granting of a TSP phase within the same cycle; the percentage of granted TSP phases that were triggered by a TSP request in the same cycle; the probability of a TSP request benefiting from a TSP phase; the percentage of granted TSP phases that were beneficial to at least one TSP request (TSP phase effectiveness); bus and passenger time savings for a granted TSP phase; other vehicles time savings and delay due to TSP phases. Because this is the first study that has utilized and integrated the bus AVL/APC data and SCATS signal phase log data, and evaluated the TSP system performance at the stop-to-stop segment level, most of the results are new to the literature. Some of the key findings are summarized below. 
The average number of TSP requests per day at each intersection from both directions is 70. The average numbers of green extension (GE) and early green (EG) phases per day are 21 and 11, respectively. However, these numbers vary significantly across intersections. For example, there are 63 TSP phases (both GE and EG) per day at the intersection of $39^{\text {th }}$ Ave. but only 5 TSP phases per day at the intersections of $26^{\text {th }}$ and $33^{\text {rd }}$ Ave. The mean durations of GE and EG phases are 7 and 11 seconds, respectively.

An average of $45 \%$ of TSP requests resulted in the granting of a TSP phase within the same cycle (29\% GE and 16\% EG). For each TSP request, the probabilities that a GE phase is granted early, on time and late are $2.5 \%, 1.5 \%$ and $25 \%$, respectively; the probabilities that an EG phase is granted early, on time and late are 5\%,10\% and 1\%, respectively. Although a TSP request is more likely to result in the granting of a GE phase $(29 \%)$ than an EG phase (16\%) within a cycle, a TSP request is less likely to benefit from a GE phase (1.5\% on-time) than from an EG phase (10\% on-time).

Assuming that the bus arrival rate at each intersection follows a uniform distribution, the theoretical probabilities that a bus TSP request can benefit from a GE phase and from an EG phase are $6 \%$ and $30 \%$, respectively. Therefore, the difference between the actual and the theoretical probabilities of a TSP request benefiting from TSP phases indicates that it is necessary to improve the TSP system performance by increasing the probability that a TSP request benefits from TSP phases and reducing the probability that a TSP request results in a TSP phase granted early or late. 
The estimated bus time savings per TSP request from a GE phase and from an EG phase are 0.3 and 0.5 seconds, respectively. Based on regression analysis on the same corridor, Albright and Figliozzi (2012a) found that the average time savings for a bus that requests TSP is 0.41 seconds, which is very close to the $0.3-0.5$ seconds time savings. The estimated total passenger time savings per TSP request from a GE phase and from an EG phases are 7.5 and 10 seconds, respectively.

For GE phases, only $5 \%$ of them were granted on time to a TSP request, $3 \%$ and $64 \%$ of the GE phases were granted early and late to a TSP request in the same cycle, and $28 \%$ of the GE phases were granted without a TSP request in the same cycle. On the other hand, the percentages of EG phases that were granted early, on-time and late to a TSP request in the same cycle are $40 \%, 40 \%$ and $8 \%$, respectively, and $12 \%$ of the EG phases were granted without a TSP request in the same cycle. These results indicate that early green effectiveness is much higher than green extension effectiveness, and too many green extension phases were granted late $(64 \%)$.

The estimated bus time savings of a GE phase and an EG phase are 1 and 4 seconds, respectively. The estimated passenger time savings of a GE phase and an EG phase are 20 and 90 seconds, respectively. Although Smith et al. (2005) suggested that a TSP system works better for far-side bus stops than near-side bus stops because it is difficult to predict bus arrival time at near-side bus stops, our results did not show clear differences in bus and passenger time savings per TSP phase between near-side stops and far-side stops. However, this does not mean that bus stop configuration has no impact on 
TSP performance because most of the evaluated near-side stop segments may have TSP setting problems.

The estimated bus passenger time savings per EG phase is less than the delay to side street vehicles at intersections with large side street traffic volumes; but the estimated passenger time savings per GE phase is less than the delay to side street vehicles at all intersections. In addition, the total time savings of bus passengers and vehicles on the major street is higher (or equal to) than the delay to side street vehicles for an EG phase (or a GE phase) at all intersections.

\subsection{Implications}

Results from the bus stop-to-stop travel time models provide valuable information for transit agencies to understand factors that affect bus travel time across intersections. The estimated signal delay and $\mathrm{RC}$ ratio coefficients provide quantitative support for transit agencies to estimate bus travel time delay at each intersection by considering intersection signal timing characteristics. The results can help transit agencies to decide: whether a near-side bus stop or a far-side bus stop should be placed at each intersection along a corridor; add or reduce slack times for time point bus stops near intersections; change intersection geometry; and propose appropriate control strategies to help reduce bus travel time delay and improve service reliability. 
The TSP performance evaluation results provide worthwhile information for cities and transit agencies to identify potential problems and improvement opportunities for the TSP system. For example, the very low numbers of TSP phases at $26^{\text {th }}, 33^{\text {rd }}$ and $50^{\text {th }}$ Ave. clearly indicate problematic TSP settings at these intersections. There is enough room to improve the probability of a TSP request benefiting from a TSP phase, because the actual probabilities are $1.5 \%$ from a GE phase and $10 \%$ from an EG, while the expected probabilities are $6 \%$ from a GE phase and $30 \%$ from an EG. If these probabilities can be improved, the expected bus time savings and passenger time savings will increase significantly, and the delay to side street traffic will decrease substantially.

The TSP phase effectiveness results show that too many green extension phases were granted either late $(64 \%)$ but still in the same cycle or in a different cycle $(28 \%)$. These results indicate potential problems with the TSP control logic or signal controllers reliability issues when receiving and clearing a TSP request. For example, a GE phase might be granted regardless of the time when a TSP request is detected. Late granted GE phases are not helpful for buses on the major street and they create additional delay to the side-street traffic. If the percent of late GE phases can be reduced significantly, the expected bus time savings and passenger time savings per granted GE phase will increase significantly.

Although this study is based on a specific urban arterial corridor where signals are operated under the SCATS system, some of the findings can be applied to other transit routes and corridors with similar roadway geometry and signal timing characteristics. For 
example, the average signal delay is about $70 \%$ of the designed red phase duration of an intersection. Also, the stop-to-stop segment level travel time regression models can be used to quantify the impacts of signal delay, traffic conditions and bus stop location for other transit routes and corridors with different roadway geometry and signal control systems. The TSP performance evaluation results are specific for this study corridor, but the algorithms and performance measures can be directly applied to any corridors as long as similar archived data sources are available. Although TSP system design and preimplementation evaluation is important, results from this study indicate that post TSP implementation performance evaluation is also necessary, because some practical problems or potential improvement opportunities may be identified.

\subsection{Recommendations}

Based on the results from this research, this study provides a few recommendations for cities and transit agencies to reduce bus travel time delay and variability, and to improve TSP system effectiveness in terms of maximizing transit vehicle time savings and minimizing side street traffic delay. Some of the recommendations include:

1. Adjust TSP settings for the intersections at $26^{\text {th }}$ Ave., $33^{\text {rd }}$ Ave. and $50^{\text {th }}$ Ave. on Powell Blvd. There might be TSP request detection problems or TSP control logic issues at these intersections. By comparing the TSP settings at these intersections with other intersections, these problems can be easily solved. 
2. Examine bus emitter activation/deactivation reliability and traffic signal controller reliability in receiving and clearing TSP requests. The TSP Opticom log data (available in the City of Portland) can be utilized to evaluate the reliability of traffic signal controllers. By integrating the TSP Opticom log data with the bus AVL/APC data, bus emitter activation and deactivation reliability can also be estimated. Once the reliability of these two sub systems can be measured and understood, more specific TSP system problems can be identified and appropriate strategies can be implemented.

3. Reduce unnecessary (late) granted green extension phases by comparing the predicted bus arrival time at the intersection when a priority request is received with the remaining time of a green phase. This can be done by adding a threshold to the TSP control logic, for example, no green extension phase should be granted if a bus TSP request is detected 20 seconds before the end of a regular green phase. However, to optimize the values of such threshold can be challenging because TSP request detection zone length and signal timing characteristics have to be considered. Depending on how TSP control logic is programmed in the SCATS system, it may or may not be difficult to achieve this.

\subsection{Contributions}

This study has three important contributions:

1. This study developed an algorithm that integrates the archived bus AVL/APC data, SCATS phase log data and intersection vehicle count data; 
2. this study simultaneously quantified the impacts of intersection signal delay, traffic conditions and bus stop location (near-side vs. far-side) on bus stop-to-stop segment travel time based on empirical data; and

3. this study proposed novel performance measures to evaluate the TSP system effectiveness at the stop-to-stop segment level.

\subsection{Future Research}

In this study, the bus AVL/APC data is collected at bus stops while the SCATS signal phase log data is collected at intersections. Because of the difference in geographic dimensions, bus queuing conditions at intersections and bus trajectories through stop-tostop segments are unknown. TriMet's new bus dispatching system provides higher resolution bus location data (vehicle positions is recorded every 5 seconds) and bus wheel movement data that records when and where buses stop and start moving along the route. These higher resolution data, together with the data analyzed in this study, provide great opportunities to better understand the queuing effect on bus travel time and more actually evaluate the TSP system performance.

The TSP evaluation results indicate some potential reliability problems of traffic signal controllers receiving and clearing TSP request calls. The City of Portland also provides TSP Opticom log data that records the intensity of TSP request calls received by traffic signal controllers. This data will provide valuable additional information to understand 
reasons that led to some of the low TSP performance and to deploy appropriate strategies to improve the TSP system performance.

This study has analyzed the impacts of intersection delay, traffic conditions and bus stop location on bus travel time variability, and has analyzed the TSP system performance on reducing bus travel time delay. It is also worthwhile to study the impacts of intersection delay, traffic conditions, bus stop location and TSP system on other bus service reliability measures such as schedule/headway delay and variation.

This study only analyzed 12 far-side segments and 6 near-side segments. There are not enough bus stop-to-stop segments to study the impacts of intersection and segment geometric characteristics on bus travel time and service reliability. Therefore, it is worthwhile to include more stop-to-stop segments. One example of the future steps will be to include stop-to-stop segments without intersections to control for the effects of bus bay on bus travel time and service reliability.

This study utilized the 15-minute traffic count data to study the impact of traffic conditions on bus travel time. However, the 15-minute aggregation level traffic volume may not be able to accurately represent the traffic conditions during the time a bus passing through the intersection. It is necessary to utilize the cycle-by-cycle traffic volume data provided by the SCATS system to more accurately analyze the impact of traffic conditions on bus travel time and service reliability. 
The TSP performance evaluation results indicate a potential control logic problem for the existing TSP system: too many unnecessary green extension phases were granted. Therefore, it is worth for transit agencies and cities to implement a real-time optimal TSP control system to maximize the benefits to transit vehicles and minimize the delay to side street vehicles. The real-time optimal TSP control system should be able to communicate with bus and signal phase status in real-time and predict bus arrival time at the intersection based on historical data and real-time bus status information. The objective function of the optimal TSP control system should consider the total passenger time savings and delay from both buses and other vehicles from all approaches of an intersection, bus schedule delay and headway to its preceding and following buses, and bus arrival time at the downstream intersection if signals are coordinated. The decisions of the TSP control system will include: whether to grant a TSP phase or not; green extension or early green phase; and when to start and end the phase. The optimal control system should update decisions whenever real-time bus information is updated. Such a real-time optimal control system is expected to improve the effectiveness and efficiency of a TSP system. 


\section{REFERENCES}

Abdy, Z.R., Hellinga, B.R., 2011. Analytical Method for Estimating the Impact of Transit Signal Priority on Vehicle Delay. Journal of Transportation Engineering 137, 589-600.

Abkowitz, M.D., 1978. Transit Service Reliability ( No. UMTA-MA-06-0049-78-1). Cambridge, MA.

Abkowitz, M.D., Eiger, A., Engelstein, I., 1986. Optimal Control of Headway Variation on Transit Routes. Journal of Advanced Transportation 20, 73-88.

Abkowitz, M.D., Engelstein, I., 1983. Factors affecting running time on transit routes. Transportation Research Part A: General 17, 107-113.

Abkowitz, M.D., Engelstein, I., 1984. Methods for Maintaining Transit Service Regularity. Transportation Research Record 961, 1-8.

Abkowitz, M.D., Lepofsky, M., 1990. Implementing Headway-based Reliability Control on Transit Routes. Journal of Transportation Engineering 116, 49-63.

Abkowitz, M.D., Tozzi, J., 1987. Research contributions to managing transit service reliability. Journal of Advanced Transportation 21, 47-65.

Albright, E., Figliozzi, M., 2012a. Factors Influencing Effectiveness of Transit Signal Priority and Late-Bus Recovery at Signalized-Intersection Level. Transportation Research Record 186-197.

Albright, E., Figliozzi, M., 2012b. Schedule recovery for late buses: What are the individual and joint contributions of Transit Signal Priority and bus operator behavior?, in: Working Paper.

Albright, E., Figliozzi, M., 2012c. Analysis of the impacts of transit signal priority on bus bunching and performance, in: Proceedings of the 12th Conference on Advanced Systems for Public Transport. Santiago, Chile.

Balke, K., Dudek, C., Urbanik II, T., 2000. Development and Evaluation of Intelligent Bus Priority Concept. Transportation Research Record 1727, 12-19.

Bertini, R.L., El-Geneidy, A.M., 2004. Modeling transit trip time using archived bus dispatch system data. Journal of Transportation Engineering 130, 56-67.

Byrne, N., Koonce, P., Bertini, R., Pangilinan, C., Lasky, M., 2005. Using Hardware-inthe-Loop Simulation to Evaluate Signal Control Strategies for Transit Signal Priority. Transportation Research Record 1925, 227-234.

Ceder, A., 2007. Public Transit Planning And Operation: Theory, Modelling And Practice. Elsevier.

Chada, S., Newland, R., 2002. Effectiveness of bus signal priority: final report ( No. NCTR-416-04).

Christofa, E., Skabardonis, A., 2011. Traffic Signal Optimization with Application of Transit Signal Priority to an Isolated Intersection. Transportation Research Record 2259, 192-201. 
Conrad, M., Dion, F., Yagar, S., 1998. Real-Time Traffic Signal Optimization with Transit Priority: Recent Advances in the Signal Priority Procedure for Optimization in Real-Time Model. Transportation Research Record 1634, 100109.

Crout, D., 2007. Accuracy and Precision of the Transit Tracker System. Transportation Research Record 1992, 93-100.

Diab, E.I., El-Geneidy, A.M., 2012. Understanding the impacts of a combination of service improvement strategies on bus running time and passenger's perception. Transportation Research Part A: Policy and Practice 46, 614-625.

Diab, E.I., El-Geneidy, A.M., 2013. Variation in bus transit service: understanding the impacts of various improvement strategies on transit service reliability. Public Transp 4, 209-231.

Dion, F., Hellinga, B., 2002. A Rule-based Real-time Traffic Responsive Signal Control System with Transit Priority: Application to An Isolated Intersection. Transportation Research Part B: Methodological 36, 325-343.

Dion, F., Rakha, H., Zhang, Y., 2004. Evaluation of Potential Transit Signal Priority Benefits along a Fixed-Time Signalized Arterial. Journal of Transportation Engineering 130, 294-303.

Dueker, K.J., Kimpel, T.J., Strathman, J.G., Callas, S., 2004. Determinants of Bus Dwell Time. Journal of Public Transportation 7, 21-40.

Eberlein, X.J., Wilson, N.H.M., Barnhart, C., 1998. The Real-Time Deadheading Problem in Transit Operations Control. Transportation Research Part B: Methodological 32, 77-100.

Eberlein, X.J., Wilson, N.H.M., Bernstein, D., 2001. The Holding Problem with RealTime Information Available. Transportation Science 35, 1-18.

EI-Geneidy, A., 2005. The Use of Advanced Information Technology in Urban Public Transportation Systems: An Evaluation of Bus Stop Consolidation (Ph.D. Dissertation). Portland State University, Portland, Oregon.

El-Geneidy, A., Strathman, J., Kimpel, T., Crout, D., 2006. Effects of Bus Stop Consolidation on Passenger Activity and Transit Operations. Transportation Research Record 1971, 32-41.

El-Geneidy, A.M., Horning, J., Krizek, K.J., 2011. Analyzing Transit Service Reliability Using Detailed Data From Automatic Vehicular Locator Systems. Journal of Advanced Transportation 45, 66-79.

El-Geneidy, A.M., Hourdos, J., Horning, J., 2009. Bus Transit Service Planning and Operations in a Competitive Environment. Journal of Public Transportation 12, 39-59.

El-Geneidy, A.M., Surprenant-Legault, J., 2010. Limited-stop bus service: an evaluation of an implementation strategy. Public Transport 2, 291-306.

Figliozzi, M., Feng, W., 2012. A study of headway maintenance for bus routes: causes and effects of "bus bunching" in extensive and congested service areas ( No. OTREC-RR-12-09). Portland State University, Portland, Oregon.

Fu, L., Liu, Q., Calamai, P., 2003. Real-Time Optimization Model for Dynamic Scheduling of Transit Operations. Transportation Research Record 1857, 48-55. 
Furth, P., Muller, T.H., 2000. Conditional Bus Priority at Signalized Intersections: Better Service with Less Traffic Disruption. Transportation Research Record 1731, 2330.

Furth, P., Rahbee, A., 2000. Optimal Bus Stop Spacing Through Dynamic Programming and Geographic Modeling. Transportation Research Record 1731, 15-22.

Furth, P.G., Hemily, B., Muller, T.H.J., Strathman, J.G., 2006. Using Archived AVLAPC Data to Improve Transit Performance and Management ( No. TCRP Report 113).

HCM, 2010. Highway Capacity Manual: Chapter 4 - Traffic Flow and Capacity Concepts, in: Highway Capacity Manual. Transportation Research Board, Washington, DC.

He, Q., Head, K.L., Ding, J., 2011. Heuristic Algorithm for Priority Traffic Signal Control. Transportation Research Record 2259, 1-7.

Hensher, D.A., Stopher, P., Bullock, P., 2003. Service quality-developing a service quality index in the provision of commercial bus contracts. Transportation Research Part A: Policy and Practice 37, 499-517.

Hunter-Zaworski, K.M., Kloos, W.C., Danaher, A.R., 1995. Bus Priority at Traffic Signals in Portland: The Powell Boulevard Pilot Project. Transportation Research Record 1503, 29-33.

Kim, S., 2013. The R Package "ppcor."

Kimpel, T., Strathman, J., Bertini, R., Callas, S., 2005. Analysis of Transit Signal Priority Using Archived TriMet Bus Dispatch System Data. Transportation Research Record 1925, 156-166.

Kimpel, T.J., 2001. Time Point-Level Analysis of Transit Service Reliability and Passenger Demand (Ph.D. Dissertation). Portland State University, Portland, Oregon.

Kimpel, T.J., Strathman, J.G., Callas, S., 2008. Improving Scheduling Through Performance Monitoring, in: Computer-Aided Systems in Public Transport. Springer Berlin Heidelberg, Berlin, Heidelberg, pp. 253-280.

Kittelson \& Associates, Inc., 2013. Transit Capacity and Quality of Service Manual. 3rd Edition. TCRP Report 165.

Koonce, P.J.V., Kloos, B., Callas, S., 2002. Bus priority at Traffic Signals in PortlandVersion 2.0: The Streamline Project. Presented at the ITE 2002 Annual Meeting and Exhibit.

Levinson, H., Zimmerman, S., Clinger, J., Rutherford, S., Smith, R., Cracknell, J., Soberman, R., 2003. Bus Rapid Transit, Volume 1: Case Studies in Bus Rapid Transit ( No. TCRP Report 90).

Levinson, H.S., 1991. Supervision Strategies for Improved Reliability of Bus Routes (NCTRP Synthesis of Transit Practice).

Li, H., Bertini, R., 2009. Assessing a Model for Optimal Bus Stop Spacing with HighResolution Archived Stop-Level Data. Transportation Research Record 2111, 24 32.

Lin, W.-H., 2002. Quantifying Delay Reduction to Buses with Signal Priority Treatment in Mixed-Mode Operation. Transportation Research Record 1811, 100-106. 
Ma, W., Liu, Y., Han, B., 2013. A rule-based model for integrated operation of bus priority signal timings and traveling speed: BUS PRIORITY SIGNAL TIMINGS. Journal of Advanced Transportation 47, 369-383.

Ma, W., Yang, X., Liu, Y., 2010. Development and Evaluation of a Coordinated and Conditional Bus Priority Approach. Transportation Research Record 2145, 49-58.

Mazloumi, E., Currie, G., Rose, G., 2010. Using GPS Data to Gain Insight into Public Transport Travel Time Variability. Journal of Transportation Engineering 136, 623-631.

Mazloumi, E., Moridpour, S., Currie, G., Rose, G., 2012. Exploring the Value of Traffic Flow Data in Bus Travel Time Prediction. Journal of Transportation Engineering $138,436-446$.

McKnight, C., Levinson, H., Ozbay, K., Kamga, C., Paaswell, R., 2004. Impact of Traffic Congestion on Bus Travel Time in Northern New Jersey. Transportation Research Record 1884, 27-35.

Milkovits, M., 2008. Modeling the Factors Affecting Bus Stop Dwell Time: Use of Automatic Passenger Counting, Automatic Fare Counting, and Automatic Vehicle Location Data. Transportation Research Record 2072, 125-130.

Mirchandani, P.B., Lucas, D.E., 2004. Integrated Transit Priority and Rail/Emergency Preemption in Real-Time Traffic Adaptive Signal Control. Journal of Intelligent Transportation Systems 8, 101-115.

Murray, A.T., Wu, X., 2003. Accessibility tradeoffs in public transit planning. Journal of Geographical Systems 5, 93-107.

Rutherford, G.S., Watkins, K.E., 2011. Measurement and Evaluation of Transit Travel Time Reliability ( No. TNW2010-10). University of Washington, Seattle, WA.

Saka, A., 2001. Model for Determining Optimum Bus-Stop Spacingin Urban Areas. Journal of Transportation Engineering 127, 195-199.

Schweiger, C., 2003. Real-Time Bus Arrival Information Systems ( No. TCRP Synthesis Report 48).

Skabardonis, A., 2000. Control Strategies for Transit Priority. Transportation Research Record: Journal of the Transportation Research Board 1727, 20-26. doi:10.3141/1727-03

Slavin, C., Feng, W., Figliozzi, M., Koonce, P., 2013. A Statistical Study of the Impacts of SCATS Adaptive Traffic Signal Control on Traffic and Transit Performance. Transportation Research Record 2356, 117-126.

Smith, H.R., Hemily, P.B., Ivanovic, M., 2005. Transit Signal Priority (TSP): A Planning and Implementation Handbook. ITS America.

Strathman, J., Dueker, K., Kimpel, T., Gerhart, R., Turner, K., Taylor, P., Callas, S., Griffin, D., Hopper, J., 1999. Automated Bus Dispatching, Operations Control, and Service Reliability: Baseline Analysis. Transportation Research Record 1666, 28-36.

Strathman, J.G., Dueker, K.J., Kimpel, T., Gerhart, R.L., Turner, K., Taylor, P., Callas, S., Griffin, D., 2000. Service Reliability Impacts of Computer-Aided Dispatching and Automatic Vehicle Location Technology: A TriMet Case Study. Transportation Quarterly 54, 85-102. 
Strathman, J.G., Hopper, J.R., 1993. Empirical analysis of bus transit on-time performance. Transportation Research Part A: Policy and Practice 27, 93-100.

Strathman, J.G., Kimpel, T.J., Callas, S., 2003. Headway Deviation Effects on Bus Passenger Loads: Analysis of Tri-Met's Archived AVL-APC Data ( No. TNW2003-01).

Strathman, J.G., Kimpel, T.J., Dueker, K.J., Gerhart, R.L., Callas, S., 2002. Evaluation of Transit Operations: Data Applications of Tri-Met's Automated Bus Dispatching System. Transportation 29, 321-345.

Sun, A., Hickman, M., 2005. The Real-Time Stop-Skipping Problem. Journal of Intelligent Transportation Systems 9, 91-109.

Sun, A., Hickman, M., 2008. The Holding Problem at Multiple Holding Stations, in: Computer-Aided Systems in Public Transport, Lecture Notes in Economics and Mathematical Systems. Springer Berlin Heidelberg, pp. 339-359.

Tétreault, P.R., El-Geneidy, A.M., 2010. Estimating bus run times for new limited-stop service using archived AVL and APC data. Transportation Research Part A: Policy and Practice 44, 390-402.

Texas Transportation Institute, 1996. Guidelines for the Location and Design of Bus Stops provides information on locating and designing bus stops in various operating environments ( No. TCRP 19).

Tirachini, A., 2013. Estimation of travel time and the benefits of upgrading the fare payment technology in urban bus services. Transportation Research Part C: Emerging Technologies 30, 239-256.

TriMet, 2013. Data Resources Derived from BDS Card Data.

Turnquist, M.A., 1981. Strategies for Improving Reliability of Bus Transit Service. Transportation Research Record 818, 7-13.

Turnquist, M.A., Blume, S.W., 1980. Evaluating Potential Effectiveness of Headway Control Strategies for Transit Systems. Transportation Research Record 746, 2529.

Vuchic, V., 2005. Urban Transit: Operations, Planning and Economics. John Wiley \& Sons.

Yagar, S., Han, B., 1994. A Procedure for Real-Time Signal Control That Considers Transit Interference and Priority. Transportation Research Part B: Methodological 28, 315-331. 


\section{APPENDIX A: Bus Travel Speed Empirical Distributions}

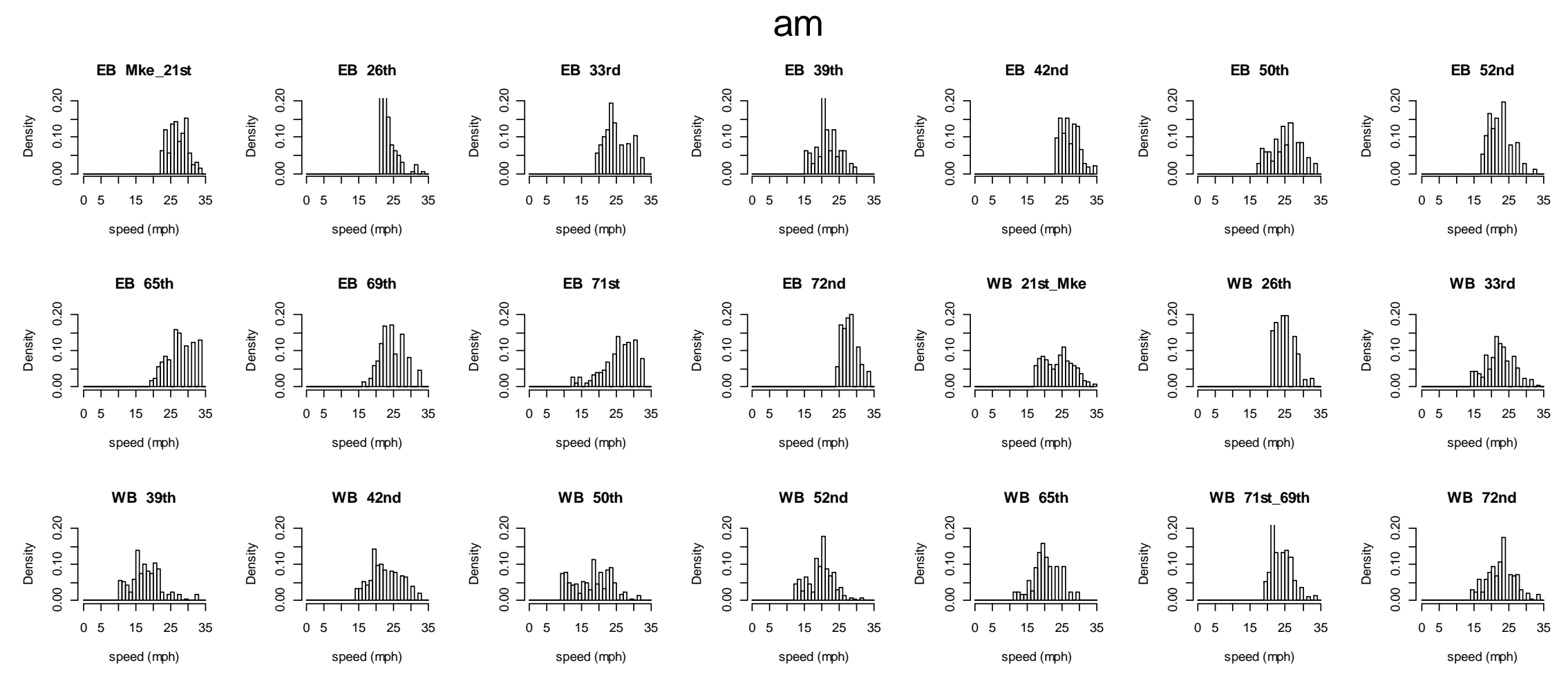



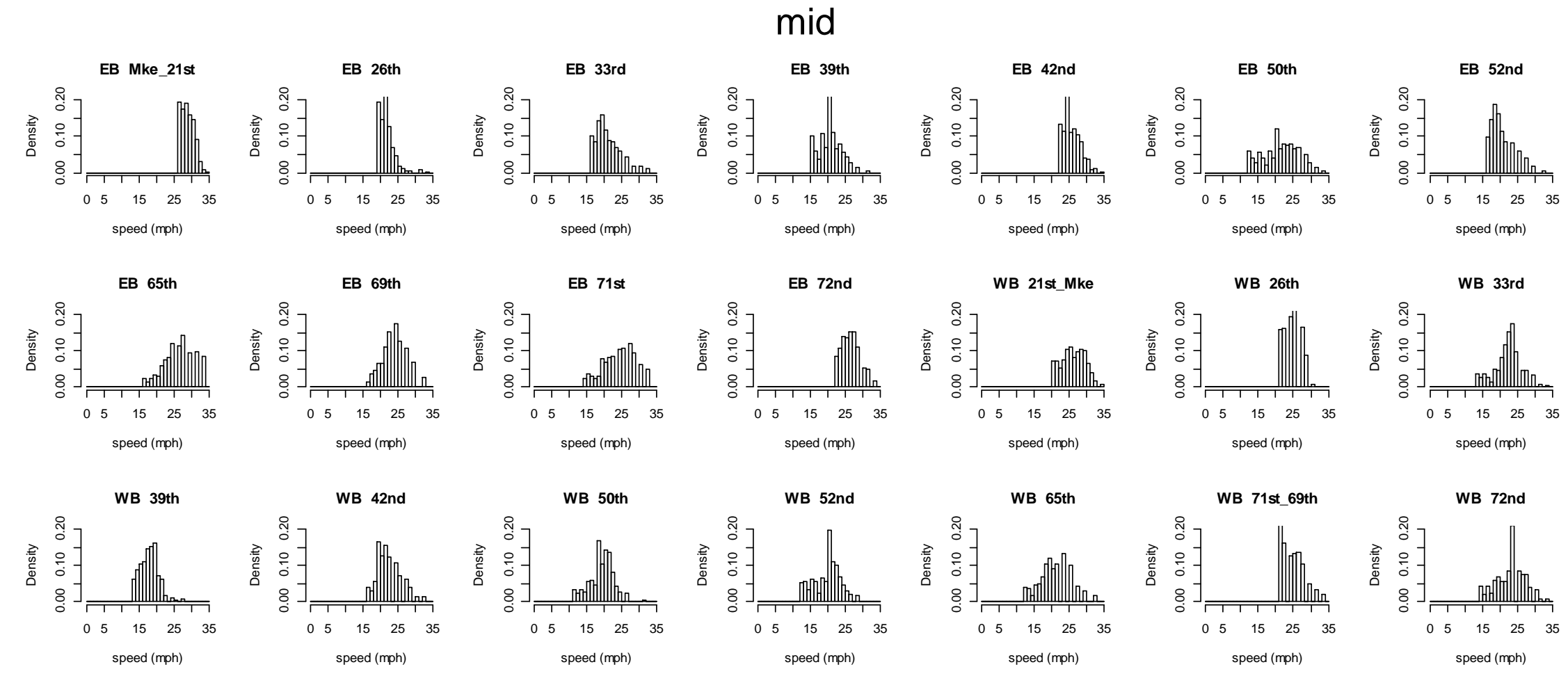

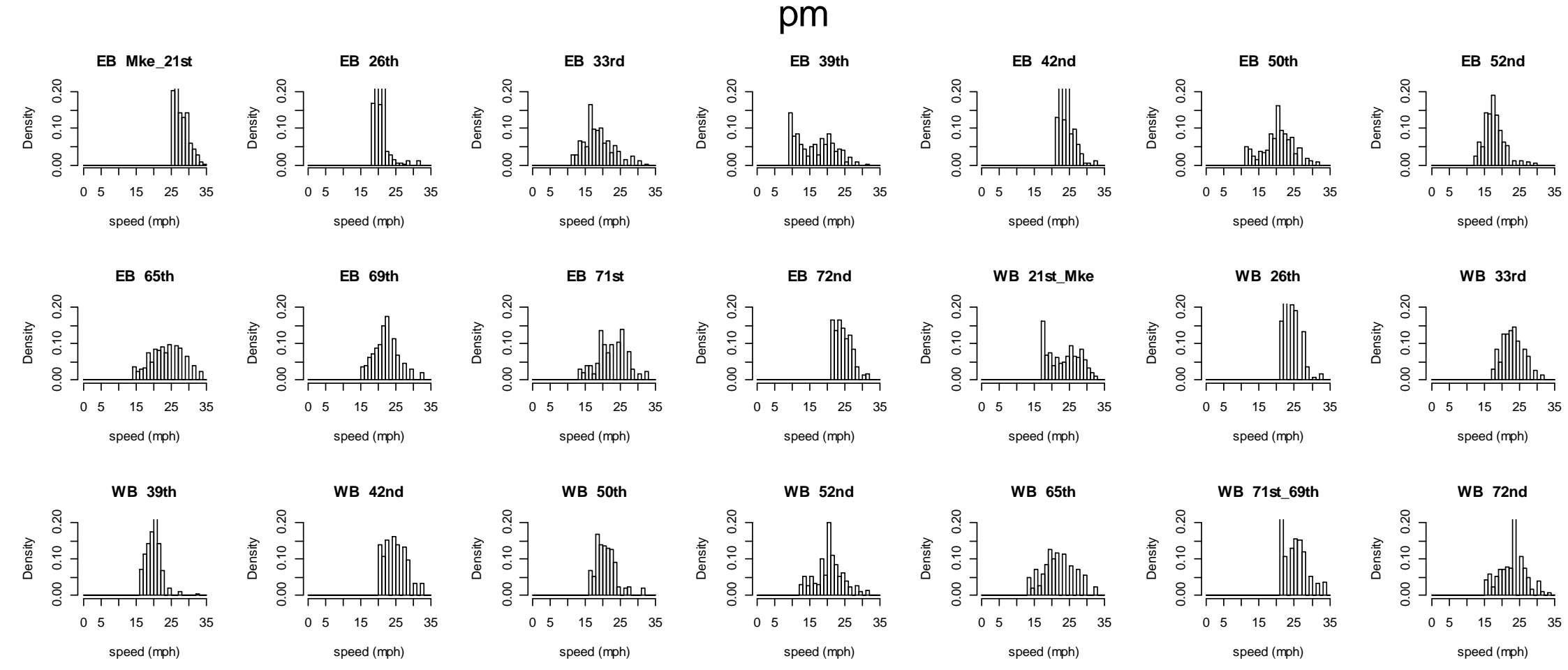

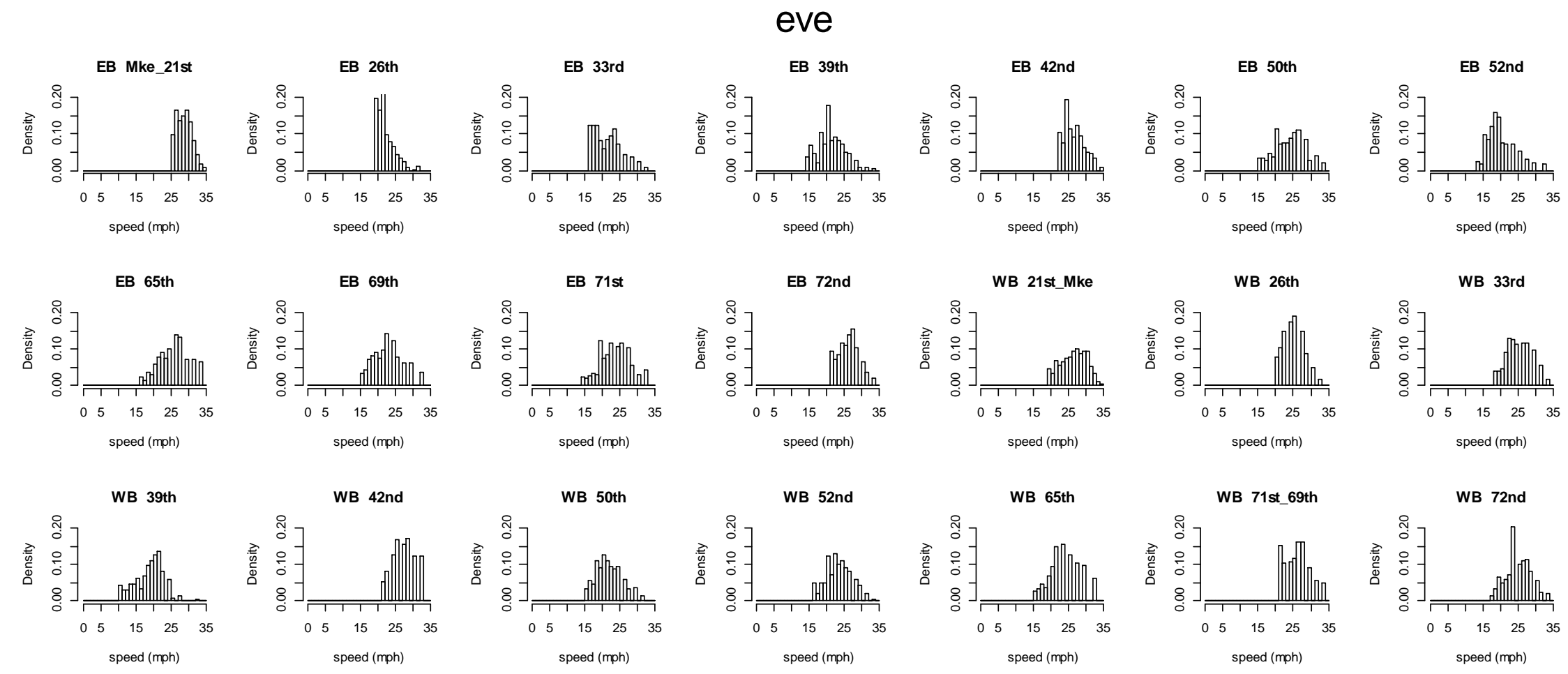


\section{APPENDIX B: Algorithm to estimate bus stop-to-stop trip signal phase attributes}

\section{Algorithm $\boldsymbol{A}$}

\section{Function}

$f(v)$ : probability density function for bus stop-to-stop travel speeds without signal delay, and $v_{\text {mean }}, v_{\min }$ and $v_{\max }$ are the mean, minimum and maximum of these speeds. Therefore, the probability of a bus traveling with a speed between $v_{1}$ and $v_{2}$ is:

$$
P\left[v_{1} \leq v<v_{2}\right]=\int_{v_{1}}^{v_{2}} f(v) \mathrm{d} v
$$

\section{Data}

$i$ : index for bus trip, $i \in \boldsymbol{I}$;

$j$ : index for signal cycle, $j \in \boldsymbol{J}$, each signal cycle is defined by the time interval between two consecutive red phase start times, and each cycle includes at least a red phase and a green phase for the bus movement direction, and might include an green extension phase and (or) early green phase;

$d_{1}, d_{2}:$ upstream distance and downstream distance;

$d t_{i}, a t_{i}:$ departure time from the departure stop and arrival time at the arrival stop for bus trip $i$;

$\operatorname{load}_{i}$ : number of onboard passengers for bus trip $i$;

$R_{j}^{S}, R_{j}^{e}$ : red phase start time and end time for cycle $j$;

$G E_{j}^{S}, G E_{j}^{e}$ : green extension phase start time and end time for cycle $j$;

$E G_{j}^{S}, E G_{j}^{e}$ : early green phase start time and end time for cycle $j$.

\section{Output}


$\operatorname{Red}_{i}$ : binary variable, it is 1 if a bus trip $i$ experienced a red signal delay at the intersection and 0 otherwise;

$\operatorname{Prob} R_{i}$ : probability of arriving at the intersection during a red phase for bus trip $i$;

Prob_G $G_{i}$ : probability of arriving at the intersection during a green phase for bus trip $i$;

Prob_GE $E_{i}$ : probability of arriving at the intersection during a green extension phase for bus trip $i$

Prob_EG $G_{i}$ : probability of arriving at the intersection during an early green phase for bus trip $i$

Exp_Delay $:$ expected red phase delay for bus trip $i$;

$B T S \_G E_{i}, P T S_{-} G E_{i}$ : expected bus (and passenger) time savings due to a green extension phase for bus trip $i$

$B T S_{-} E G_{i}, P T S_{-} E G_{i}$ : expected bus (and passenger) time savings due to an early green phase for bus trip $i$. 


\section{START $\boldsymbol{A}$}

$\operatorname{Red}_{i}=$ Prob_Red $_{i}=\operatorname{Exp}_{-}$Delay $_{i}=$ Prob_Green $_{i}=$ Prob_GE$_{i}=$ Prob_EG $_{i}=0$;

$\forall i \in \boldsymbol{I}$.

for each $j \in J$,

$\boldsymbol{I}_{j}^{\prime} \leftarrow\left\{i \mid i \in \boldsymbol{I}, R_{j}^{s} \leq d t_{i}+\frac{d_{1}}{v_{\text {mean }}} \leq R_{j}^{e}\right\}$

$\boldsymbol{I}_{j}^{\prime}$ is the set of bus trips that could encounter a red signal delay at the intersection during cycle $j . R_{j}^{S} \leq d t_{i}+\frac{d_{1}}{v_{\text {mean }}} \leq R_{j}^{e}$ means that a bus arrives at the intersection during a red phase.

if $\boldsymbol{I}_{j}^{\prime} \neq \varnothing$,

for each $i \in I_{j}^{\prime}$,

$$
\operatorname{Red}_{i}=1
$$

end for.

\section{end if.}

$$
\boldsymbol{I}_{j} \leftarrow\left\{i \mid i \in \boldsymbol{I}, R_{j}^{e}+\frac{d_{2}}{v_{\max }}<a t_{i} \leq R_{j+1}^{e}+\frac{d_{2}}{v_{\max }}\right\}
$$

$\boldsymbol{I}_{j}$ is the set of bus trips that could arrive at the arrival stop during cycle $j$.

for each $i \in \boldsymbol{I}_{j}$,

$$
t_{l}=\max \left\{a t_{i}-\frac{d_{2}}{v_{\min }}, d t_{i}+\frac{d_{1}}{v_{\max }}\right\}
$$

This is the earliest time that bus $i$ could arrive at the intersection.

$$
t_{r}=\min \left\{a t_{i}-\frac{d_{2}}{v_{\max }}, d t_{i}+\frac{d_{1}}{v_{\min }}, R_{j+1}^{S}\right\}
$$

This is the latest time that bus $i$ could arrive at the intersection.

$$
\boldsymbol{J}_{i}^{\prime} \leftarrow\left\{k \mid k \leq j, \quad d t_{i} \leq G_{j}^{e}-\frac{d_{1}}{v_{\max }}\right\}
$$

$\boldsymbol{J}_{i}^{\prime}$ is the set of cycles that bus $i$ could have spanned.

for each $j \in \boldsymbol{J}_{i}^{\prime}$,

Prob_ $R_{i}$ can be estimated by the sum of probabilities of speeds that could lead bus $i$ arrive at the intersection during the red phase $P\left[\frac{d_{1}}{R_{j}^{e}-d t_{i}} \leq v<\right.$ $\left.\frac{d_{1}}{\max \left\{R_{j}^{S}, t_{l}\right\}-d t_{i}}\right]$, conditional on the sum of probabilities of all feasible speeds

$$
\begin{aligned}
{\left[\frac{d_{1}}{t_{r}-d t_{i}} \leq\right.} & \left.v<\frac{d_{1}}{t_{l}-d t_{i}}\right] . \\
& P r o b \_R_{i} \leftarrow P r o b \_R_{i}+\frac{P\left[\frac{d_{1}}{R_{j}^{e}-d t_{i}} \leq v<\frac{d_{1}}{\max \left\{R_{j}^{S}, t_{l}\right\}-d t_{i}}\right]}{P\left[\frac{d_{1}}{t_{r}-d t_{i}} \leq v<\frac{d_{1}}{t_{l}-d t_{i}}\right]}
\end{aligned}
$$


To estimate Exp_Delay ${ }_{i}$, for any speed that could lead bus i arrive at the intersection during the red phase, $v \in\left(\frac{d_{1}}{\max \left\{R_{j}^{s}, t_{l}\right\}-d t_{i}}, \frac{d_{1}}{R_{j}^{e}-d t_{i}}\right)$, the red signal delay is: $R_{j}^{e}-\left(d t_{i}+\frac{d_{1}}{v}\right)$. Given the speed probability density function, $f(v)$,

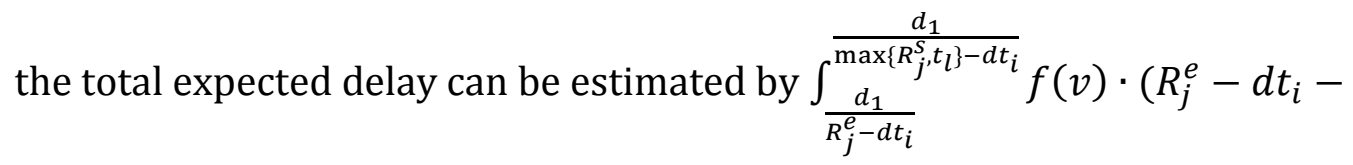
$\left.\frac{d_{1}}{v}\right) \mathrm{d} v$, conditional on the sum of probabilities of all feasible speeds $\left[\frac{d_{1}}{t_{r}-d t_{i}} \leq v<\frac{d_{1}}{t_{l}-d t_{i}}\right]$

$$
\text { Exp_Delay }_{i} \leftarrow \text { Exp_Delay }_{i}+\frac{\int_{\frac{d_{1}}{R_{j}^{e}-d t_{i}}}^{\frac{d_{1}}{\max \left\{R_{j}^{s}, t_{l}\right\}-d t_{i}}} f(v) \cdot\left(R_{j}^{e}-d t_{i}-\frac{d_{1}}{v}\right) \mathrm{d} v}{P\left[\frac{d_{1}}{t_{r}-d t_{i}} \leq v<\frac{d_{1}}{t_{l}-d t_{i}}\right]}
$$

Prob_G $G_{i}$ can be estimated similarly to Prob_R $R_{i}$ :

$$
P_{0} b_{-} G_{i} \leftarrow P r o b_{-} G_{i}+\frac{P\left[\frac{d_{1}}{\min \left\{G_{j}^{e}, t_{r}\right\}-d t_{i}} \leq v<\frac{d_{1}}{G_{j}^{s}-d t_{i}}\right]}{P\left[\frac{d_{1}}{t_{r}-d t_{i}} \leq v<\frac{d_{1}}{t_{l}-d t_{i}}\right]}
$$

if $E G_{j}^{s} \neq \varnothing$ or $E G_{j}^{e} \neq \varnothing$, which means if there is an early green phase,

Depending on the relationships between $t_{l}, E G_{j}^{s}, t_{r}$ and $E G_{j}^{e}$, the range of speeds that could lead bus $i$ arrive at the intersection during the early green phase is $\left(\max \left\{E G_{j}^{s}, t_{l}\right\}, \min \left\{E G_{j}^{e}, t_{r}\right\}\right)$; therefore, Prob_EG $G_{i}$ can be estimated by:

$P r o b \_E G_{i} \leftarrow P r o b \_E G_{i}+\frac{P\left[\frac{d_{1}}{\min \left\{E G_{j}^{e}, t_{r}\right\}-d t_{i}} \leq v<\frac{d_{1}}{\max \left\{E G_{j}^{s}, t_{l}\right\}-d t_{i}}\right]}{P\left[\frac{d_{1}}{t_{r}-d t_{i}} \leq v<\frac{d_{1}}{t_{l}-d t_{i}}\right]}$

To estimate $B T S_{-} E G_{i}$, if $E G_{j}^{S}<t_{l}$, bus $i$ is impossible to arrive at the intersection during the preceding red phase of this early green phase, the time saving for bus $i$ traveling at speed $v \in\left(\frac{d_{1}}{t_{l}-d t_{i}}, \frac{d_{1}}{\min \left\{E G_{j}^{e}, t_{r}\right\}-d t_{i}}\right)$ is $\min \left\{E G_{j}^{e}, t_{r}\right\}-$ $\left(d t_{i}+\frac{d_{1}}{v}\right)$. If $E G_{j}^{s} \geq t_{l}$, bus $i$ is also likely to arrive at the intersection during the preceding red phase of this early green phase. Therefore, time saving for 
bus $i$ traveling at speed $v \in\left(\frac{d_{1}}{E G_{j}^{s}-d t_{i}}, \frac{d_{1}}{t_{l}-d t_{i}}\right)$ is $E G_{j}^{e}-E G_{j}^{s}$. Therefore, $B T S_{-} E G_{i}$ can be estimated by:

$$
B T S_{E G_{i}} \leftarrow B T S_{E G_{i}}+
$$

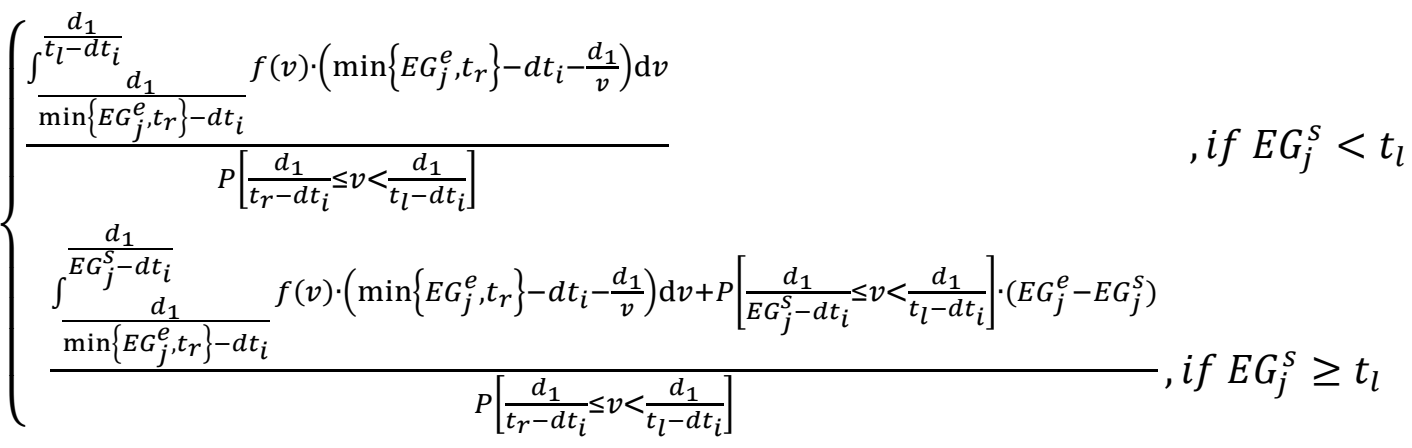

$$
\begin{aligned}
& P T S_{-} E G_{i} \leftarrow P T S_{-} E G_{i}+B T S_{-} E G_{i} \cdot \operatorname{load}_{i}
\end{aligned}
$$

\section{end if.}

if $G E_{j}^{S} \neq \varnothing$ or $G E_{j}^{e} \neq \varnothing$, which means if there is a green extension phase, Because bus $i$ could benefit from a green extension phase only when it arrives at the intersection during the green extension phase, depending on the relationships between $t_{l}, G E_{j}^{S}, t_{r}$ and $G E_{j}^{e}$, bus $i$ will benefit from GE phase if it travels with speed $v \in\left(\frac{d_{1}}{\min \left\{G E_{j}^{e}, t_{r}\right\}-d t_{i}}, \frac{d_{1}}{\max \left\{G E_{j}^{s}, t_{l}\right\}-d t_{i}}\right)$, therefore:

$$
\begin{aligned}
& \text { Prob_GE } E_{i} \leftarrow \text { Prob_GE }
\end{aligned}
$$

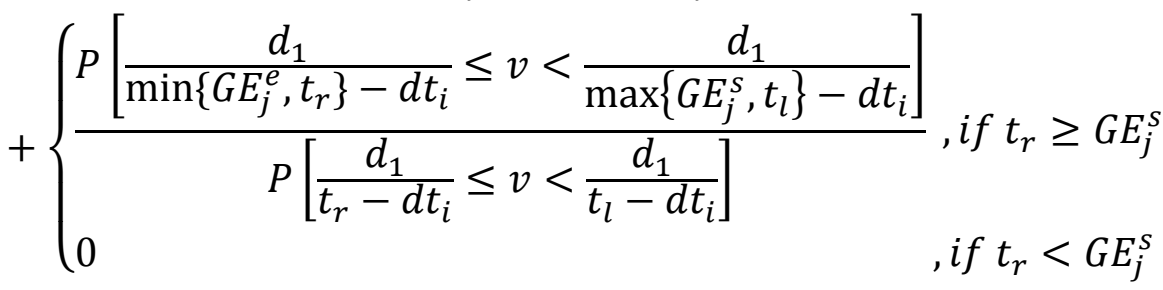

If bus $i$ travels with speed $v \in\left(\frac{d_{1}}{\min \left\{G E_{j}^{e}, t_{r}\right\}-d t_{i}}, \frac{d_{1}}{\max \left\{G E_{j}^{s}, t_{l}\right\}-d t_{i}}\right)$, the time saving is $R_{j+1}^{e}-\left(d t_{i}+\frac{d_{1}}{v}\right)$; therefore, $B T S_{-} G E_{i}$ can be estimated by:

$$
B T S_{G E_{i}} \leftarrow B T S_{G E_{i}}+
$$




$$
\begin{cases}\frac{\frac{d_{1}}{\max \left\{G E_{j}^{s}, t_{l}\right\}-d t_{i}} f(v) \cdot\left(R_{j+1}^{e}-d t_{i}-\frac{d_{1}}{v}\right) \mathrm{d} v}{\frac{d_{1}}{\min \left\{G E_{j}^{e}, t_{r}\right\}-d t_{i}}} & \text {, if } t_{r} \geq G E_{j}^{s} \\ P\left[\frac{d_{1}}{t_{r}-d t_{i}} \leq v<\frac{d_{1}}{t_{l}-d t_{i}}\right] & \text {, if } t_{r}<G E_{j}^{s} \\ & P T S_{-} G E_{i} \leftarrow P T S_{-} G E_{i}+B T S_{-} G E_{i} \cdot \operatorname{load}_{i}\end{cases}
$$

end if. end for.

end for.

end for.

end $A$. 